Supporting Information

\title{
Sulfone "Geländer" Helices: Revealing unexpected parameters controlling the enantiomerization process
}

Rajesh Mannancherry, ${ }^{[a]}$ Tomáš Šolomek, ${ }^{\ddagger[a]}$ Diana Cavalli, ${ }^{[a]}$ Juraj Malinčík, ${ }^{[a]}$ Daniel Häussinger, ${ }^{[a]}$ Alessandro Prescimone, ${ }^{[a]}$ Marcel Mayor *[a,b,c]

[a] Department of Chemistry

University of Basel

St. Johanns-Ring 19

4056 Basel, Switzerland

E-mail: marcel.mayor@unibas.ch

[b] Institute for Nanotechnology (INT)

Karlsruhe Institute of Technology (KIT)

P. O. Box 3640

76021 Karlsruhe, Germany

[c] Lehn Institute of Functional Materials (LIFM)

School of Chemistry

Sun Yat-Sen University (SYSU)

Guangzhou 510275, China

‡ R.M. and T.Š. contributed equally to this work. 


\section{Table of Contents}

HPLC Chromatograms of the Geländer Systems $\mathbf{1 b}$ and $\mathbf{2 b}$.......................................... 3

$\operatorname{NMR}\left(\mathrm{CD}_{2} \mathrm{Cl}_{2}, 600 / 151 \mathrm{MHz}, 90^{\circ} \mathrm{C}\right)$ and HR-ESI spectra of compound $2 \mathrm{a} \ldots \ldots \ldots \ldots \ldots \ldots \ldots \ldots . . . . . . . . . . . . .54$

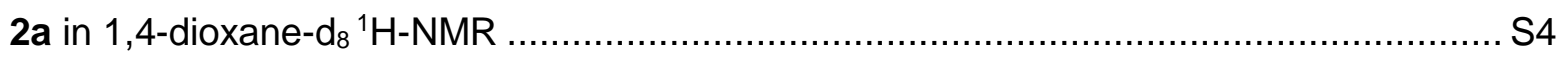

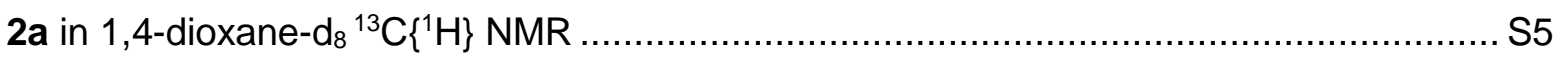

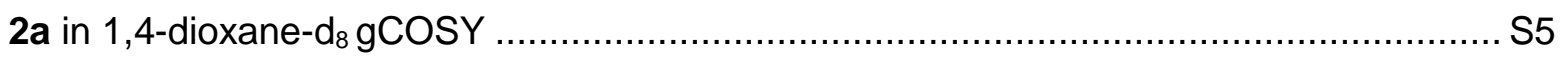

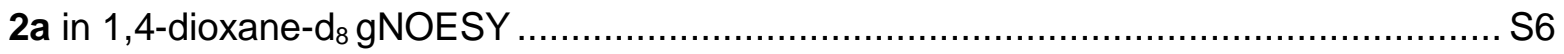

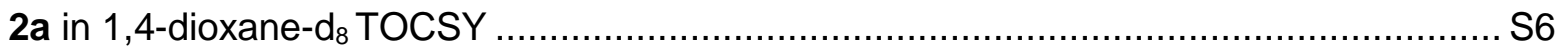

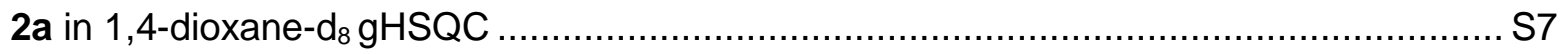

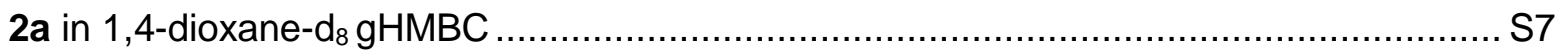

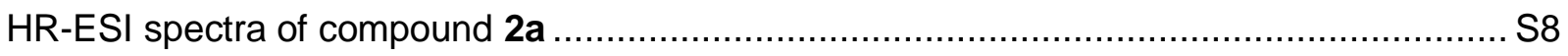

$\operatorname{NMR}\left(\mathrm{CD}_{2} \mathrm{Cl}_{2}, 600 / 151 \mathrm{MHz}, 25^{\circ} \mathrm{C}\right)$ and HR-ESI spectra of compound $1 \mathrm{a} \ldots \ldots \ldots \ldots \ldots \ldots . . . . . . . . . . . .11$

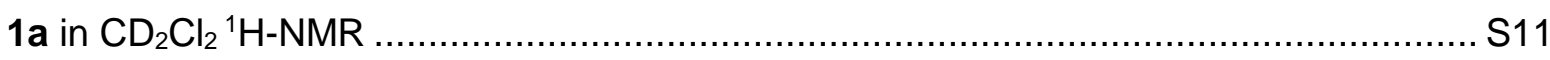

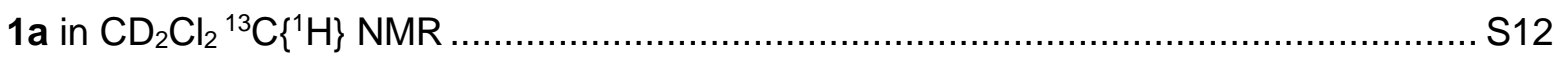

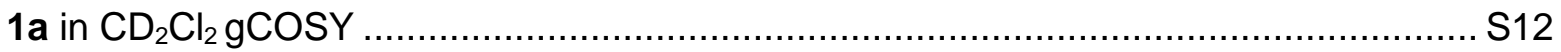

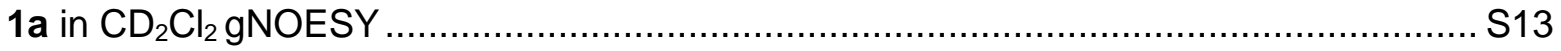

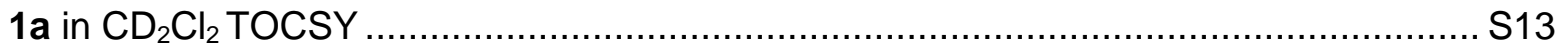

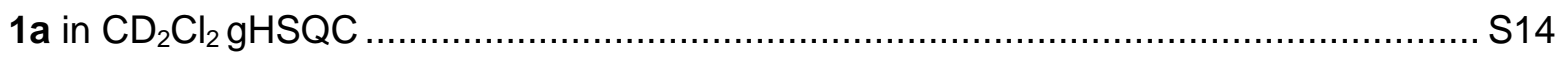

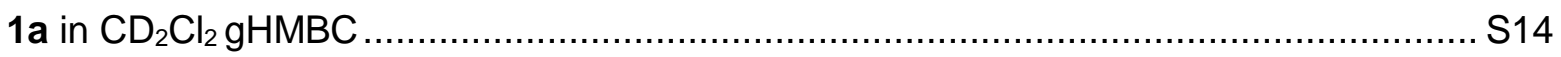

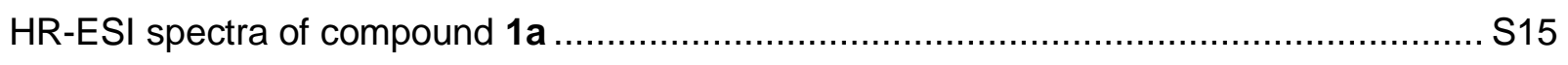

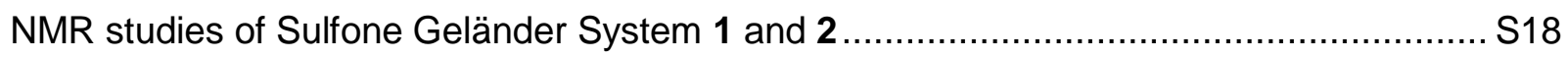

Experimental UV-Vis and CD: [12:14] Sulfon Geländer 2a ........................................... S21

Experimental UV-Vis and CD: [13:13] Sulfon Geländer 1a ......................................... S27

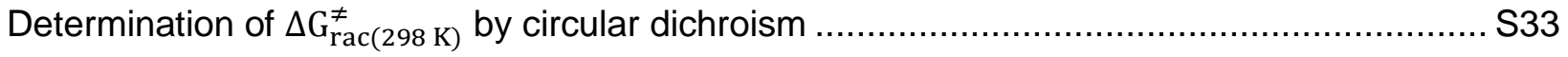

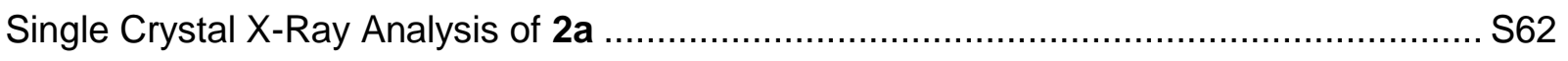

Determination of the torsion angles of the Geländer macrocycles ................................... S63

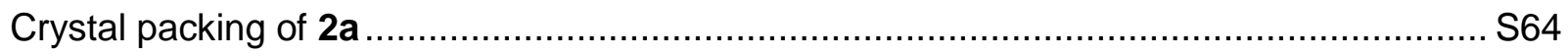

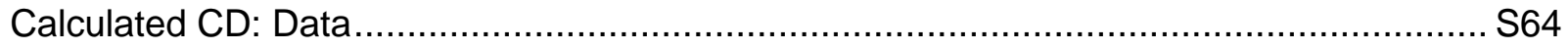

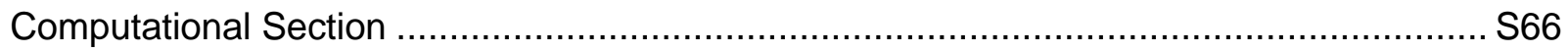

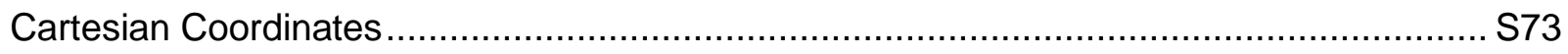

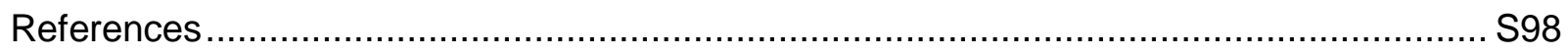




\section{HPLC Chromatograms of the Geländer Systems $1 b$ and 2b}

High-performance liquid chromatography (HPLC) using a preparative reversed stationary phase (Reprosil C18, MeCN, $30 \mathrm{~mL} \mathrm{~min}^{-1}, 25^{\circ} \mathrm{C}$ ) allowed the successful baseline separation of both structural isomers $\mathbf{1 b}$ and $\mathbf{2} \mathbf{b}$ (Figure $\mathrm{S} 1){ }^{1}$

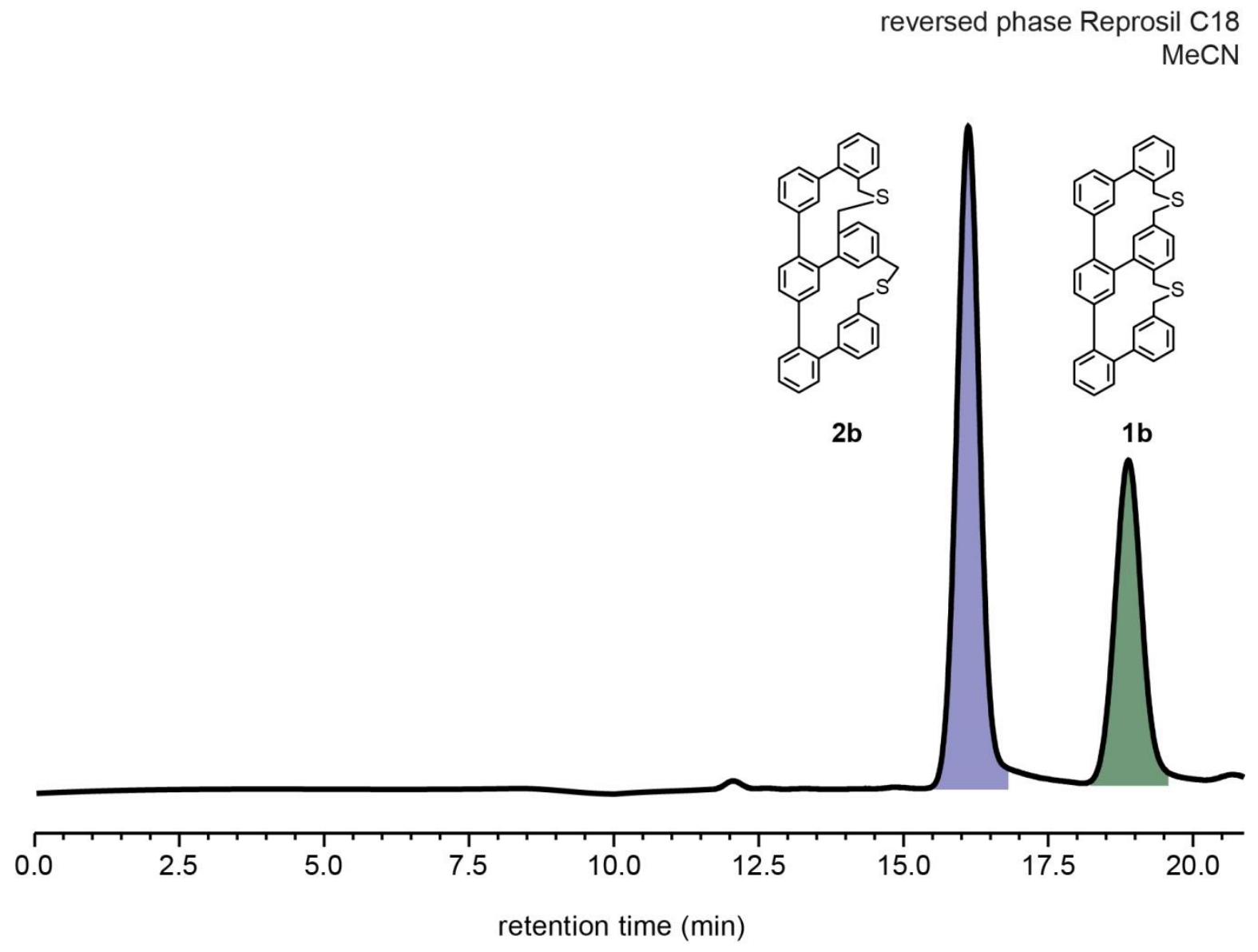

Figure S1: HPLC chromatogram of a preparative reversed (Reprosil C18, MeCN, $30 \mathrm{~mL} \mathrm{~min}^{-1}, 25^{\circ} \mathrm{C}$ ) stationary phase. Separation of both structural isomers $\mathbf{1} \mathbf{b}$ (green) and $\mathbf{2} \mathbf{b}$ (violet) on a reversed stationary phase in MeCN. 
NMR $\left(\mathrm{CD}_{2} \mathrm{Cl}_{2}, 600 / 151 \mathrm{MHz}, 90^{\circ} \mathrm{C}\right)$ and HR-ESI spectra of compound $2 \mathrm{a}$

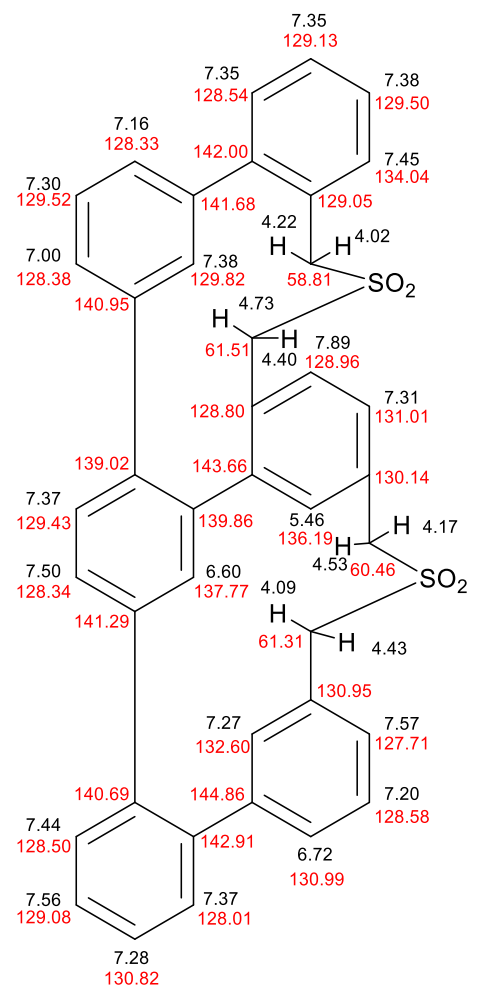

\section{2a in 1,4-dioxane-d $8{ }^{1} \mathrm{H}-\mathrm{NMR}$}

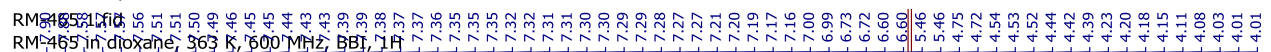

(1)
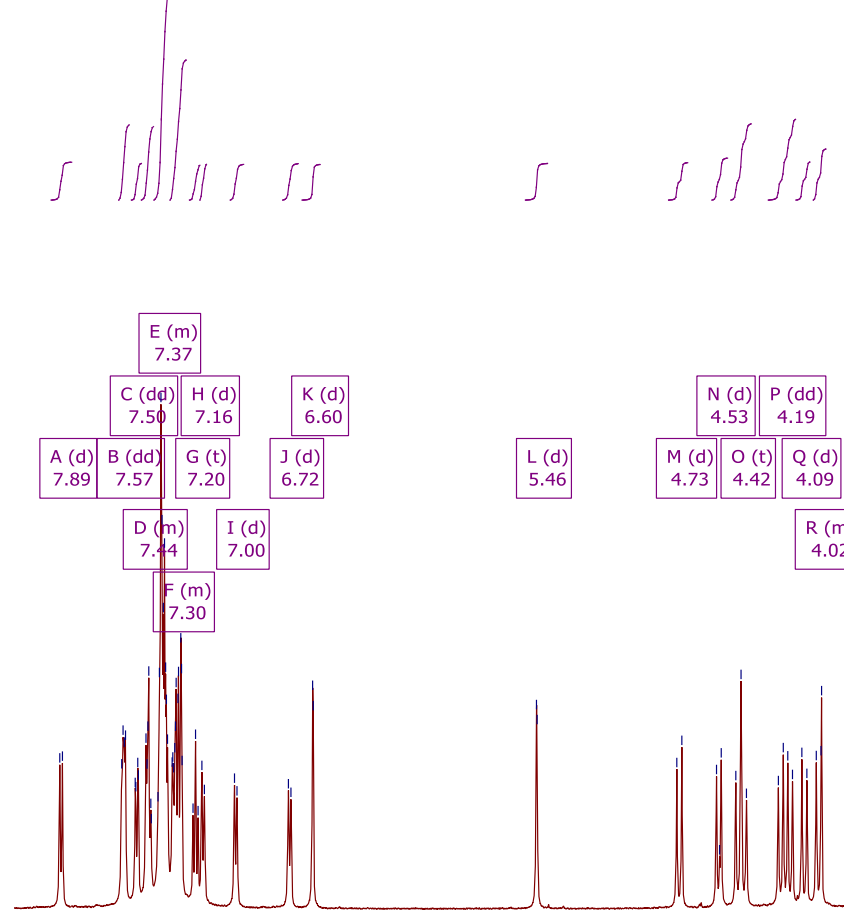

$1 \mathrm{H} \mathrm{NMR}\left(600 \mathrm{MHz},_{1 \cdot 4}\right.$-Dioxane- $\left.d_{8}\right) \delta=7.89(\mathrm{~d}, J=8.1,1 \mathrm{H}), 7.57(\mathrm{dd}, J=7.7,3.9$, $2 \mathrm{H}), 7.50(\mathrm{dd}, J=7.8,1.9,1 \mathrm{H}), 7.48-7.42(\mathrm{~m}, 2 \mathrm{H}), 7.41-7.33(\mathrm{~m}, 6 \mathrm{H}), 7.33$ $7.25(\mathrm{~m}, 4 \mathrm{H}), 7.20(\mathrm{t}, J=7.7,1 \mathrm{H}), 7.16(\mathrm{~d}, J=7.6,1 \mathrm{H}), 7.00(\mathrm{~d}, J=7.6,1 \mathrm{H}), 6.72$ $(\mathrm{d}, J=7.7,1 \mathrm{H}), 6.60(\mathrm{~d}, J=1.9,1 \mathrm{H}), 5.46(\mathrm{~d}, J=2.0,1 \mathrm{H}), 4.73(\mathrm{~d}, J=15.4,1 \mathrm{H})$, $4.53(\mathrm{~d}, J=14.4,1 \mathrm{H}), 4.42(\mathrm{t}, J=16.3,2 \mathrm{H}), 4.19$ (dd, $J=28.5,14.7,2 \mathrm{H}), 4.09$ (d, $J=15.4,1 \mathrm{H}), 4.05-3.98(\mathrm{~m}, 1 \mathrm{H})$.
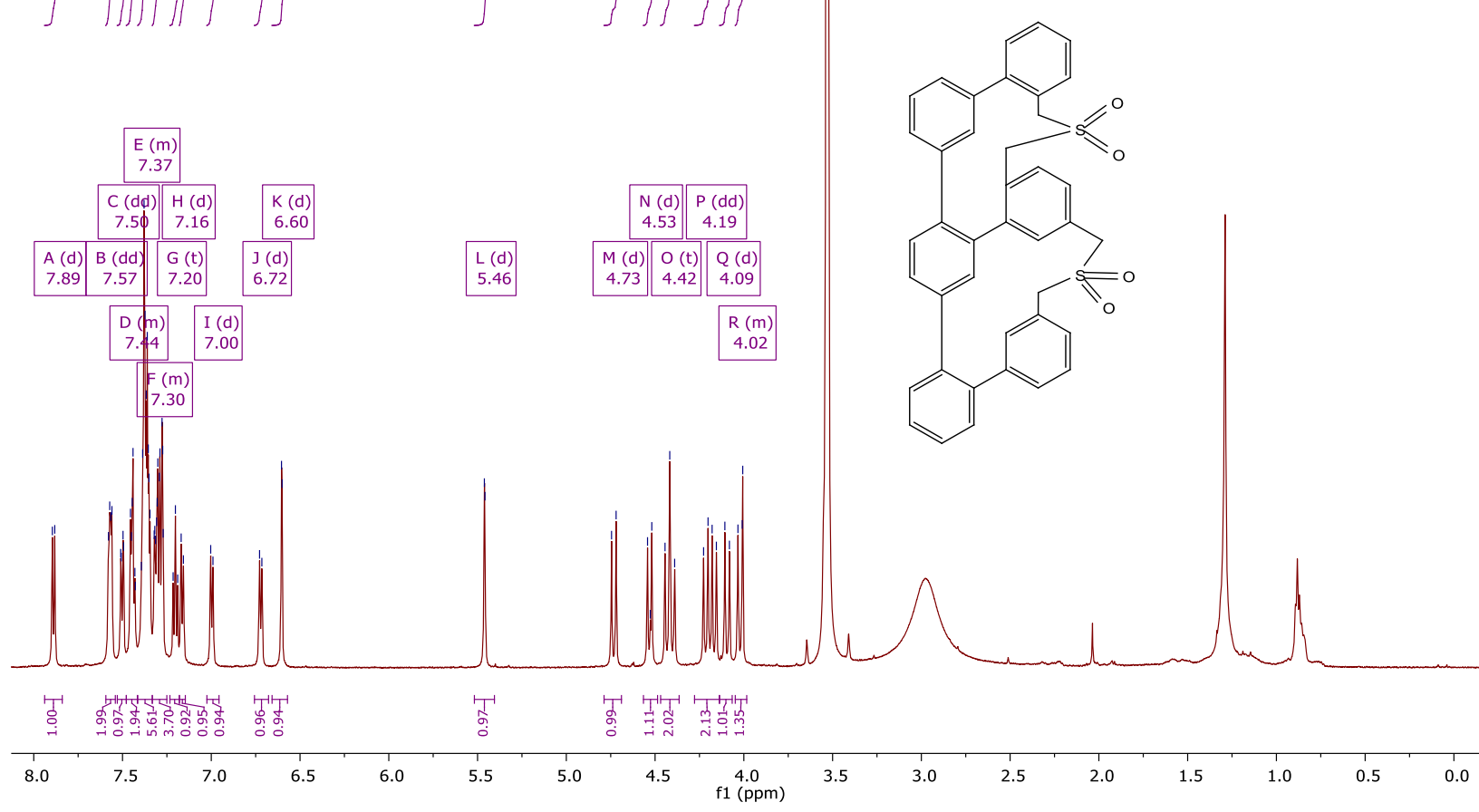
$2 \mathrm{a}$ in 1,4-dioxane-d ${ }_{8}{ }^{13} \mathrm{C}\left\{{ }^{1} \mathrm{H}\right\}-N M R$

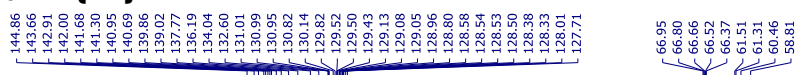

13C NMR (151 MHz, Dioxane) $8144.86,143.66,142.91,142.00,141.68$

$141.30,140.95,140.69,139.86,139.02,137.77,136.19,134.04,132.60$

$131.01,130.99,130.95,130.82,130.14,129.82,129.52,129.50,129.43$

$129.13,129.08,129.05,128.96,128.80,128.58,128.54,128.53,128.50$

$128.38,128.33,128.01,127.71,61.51,61.31,60.46,58.81$

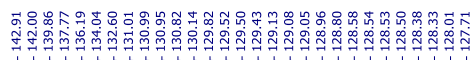

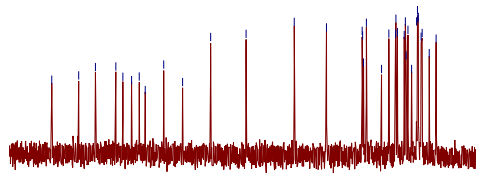

$\begin{array}{lllllllllll}146 & 144 & 142 & 140 & 138 & 136 & 134 & 132 & 130 & 128 & 126\end{array}$
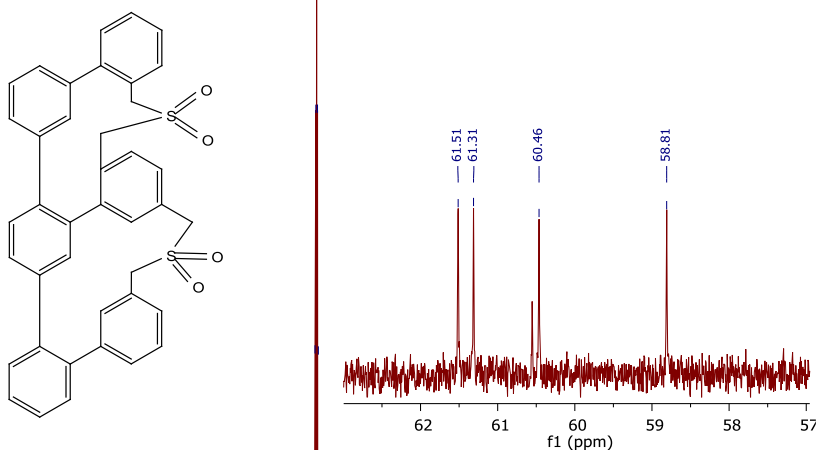

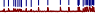

\begin{tabular}{lllllllllllllllllllllllllllll}
\hline 20 & 210 & 200 & 190 & 180 & 170 & 160 & 150 & 140 & 130 & 120 & 110 & 100 & 90 & 80 & 70 & 60 & 50 & 40 & 30 & 20 & 10 & 0 & -10
\end{tabular}

\section{$2 a$ in $1,4-$ dioxane-d $_{8}$ gCOSY}

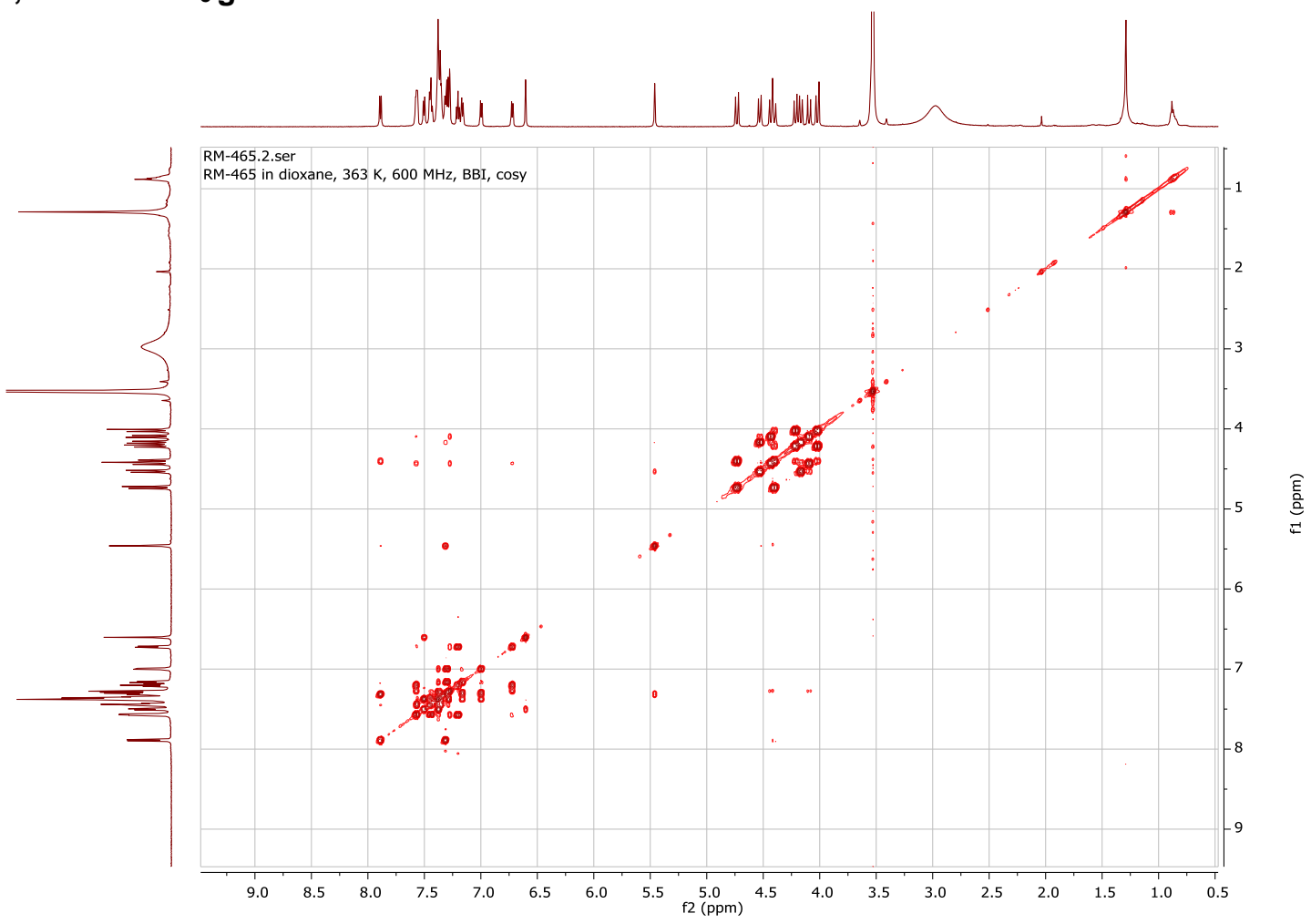


$2 a$ in 1,4-dioxane-d 8 gNOESY

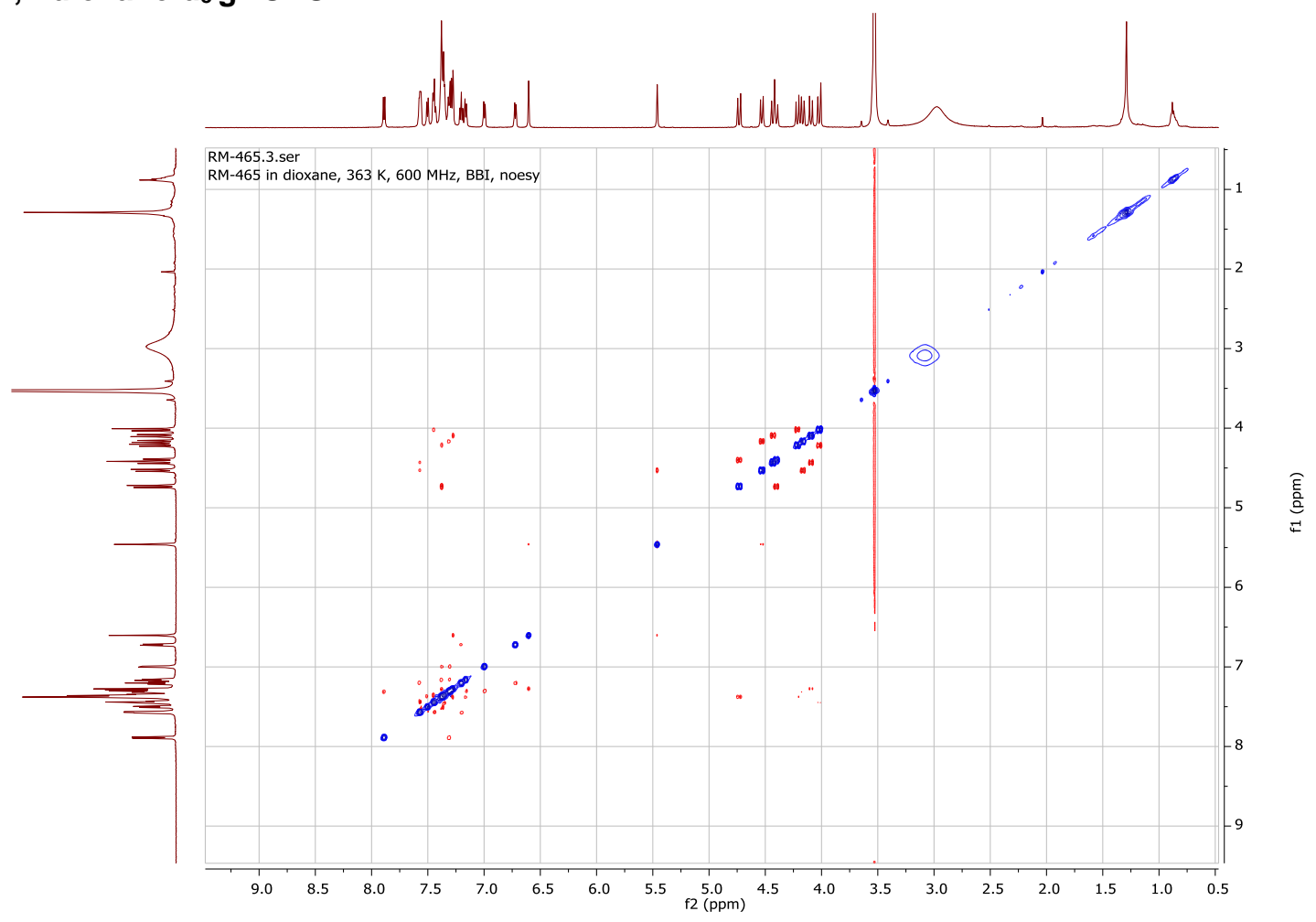

$2 a$ in 1,4-dioxane- $d_{8}$ TOCSY

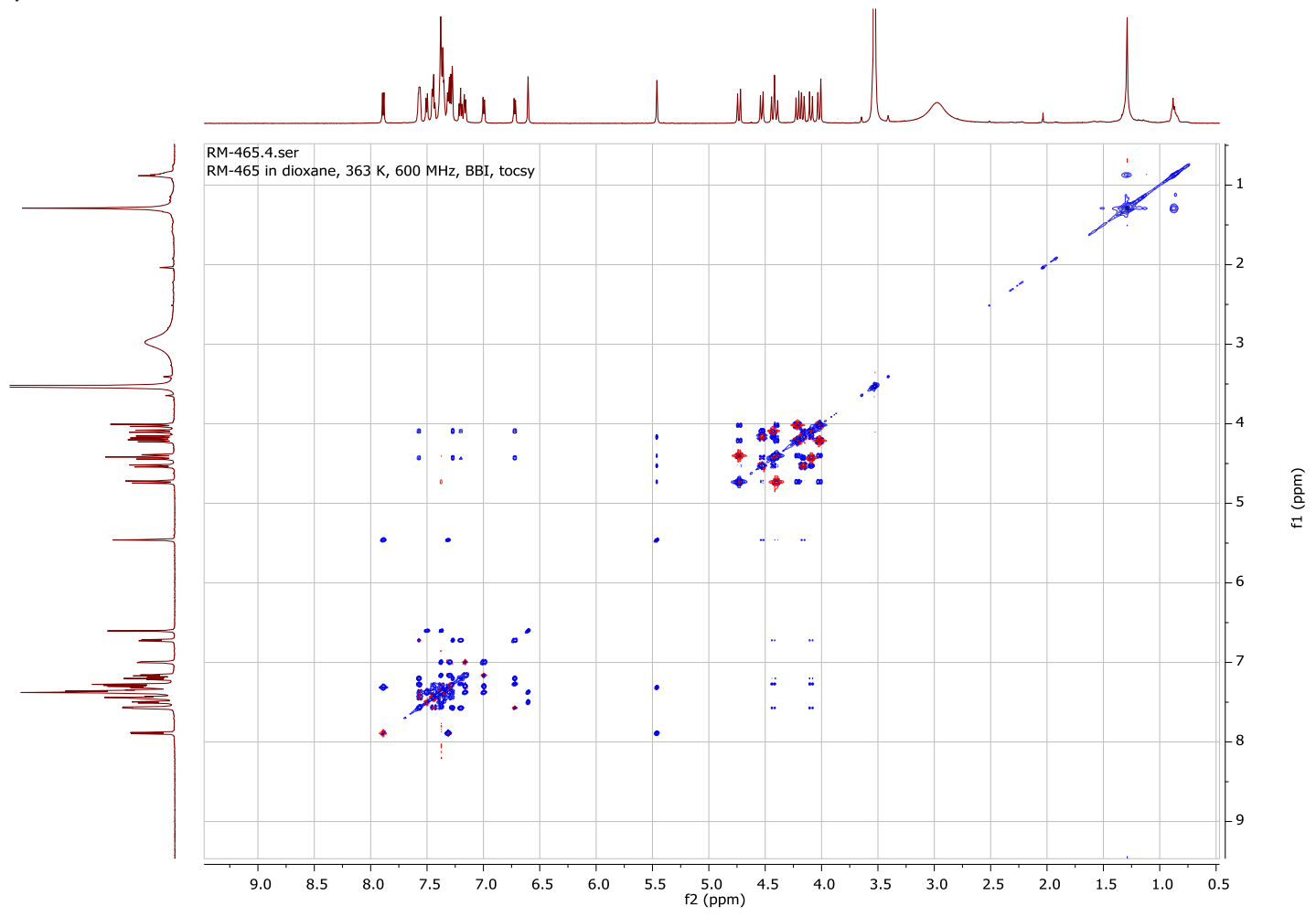


$2 a$ in $1,4-$ dioxane- $_{8}$ gHSQC

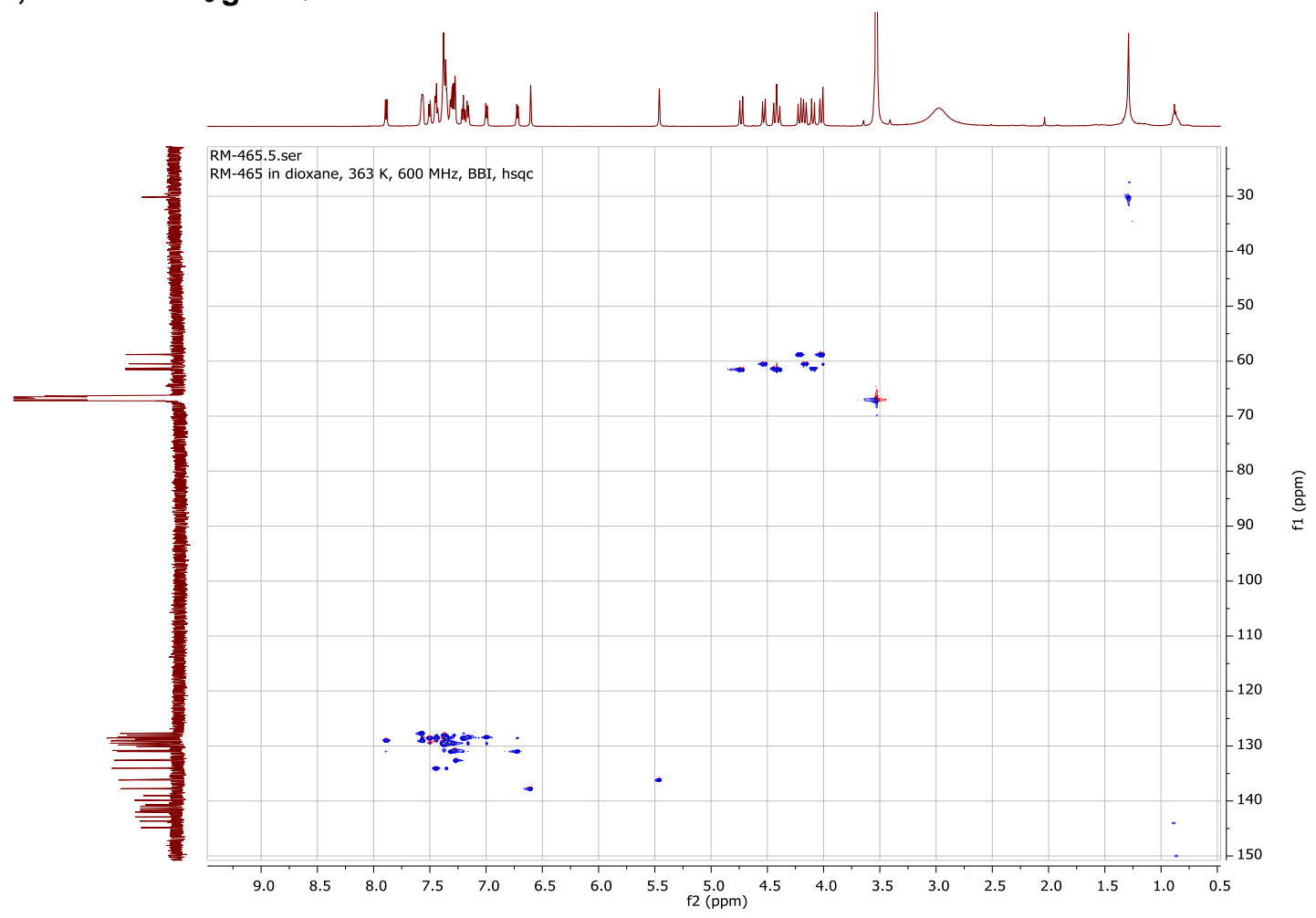

$2 a$ in 1,4 -dioxane- $d_{8}$ gHMBC

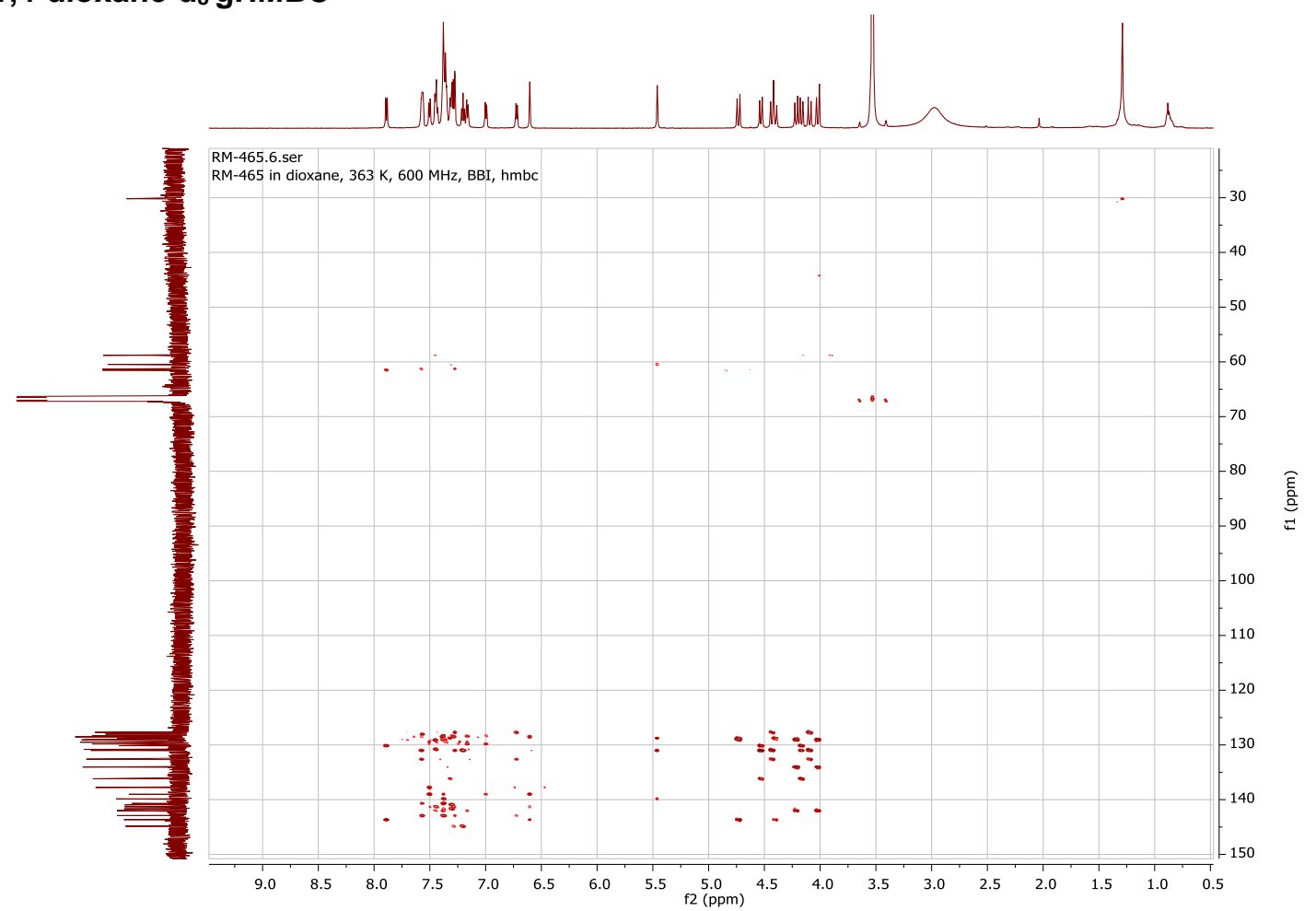




\section{HR-ESI spectra of compound 2a}

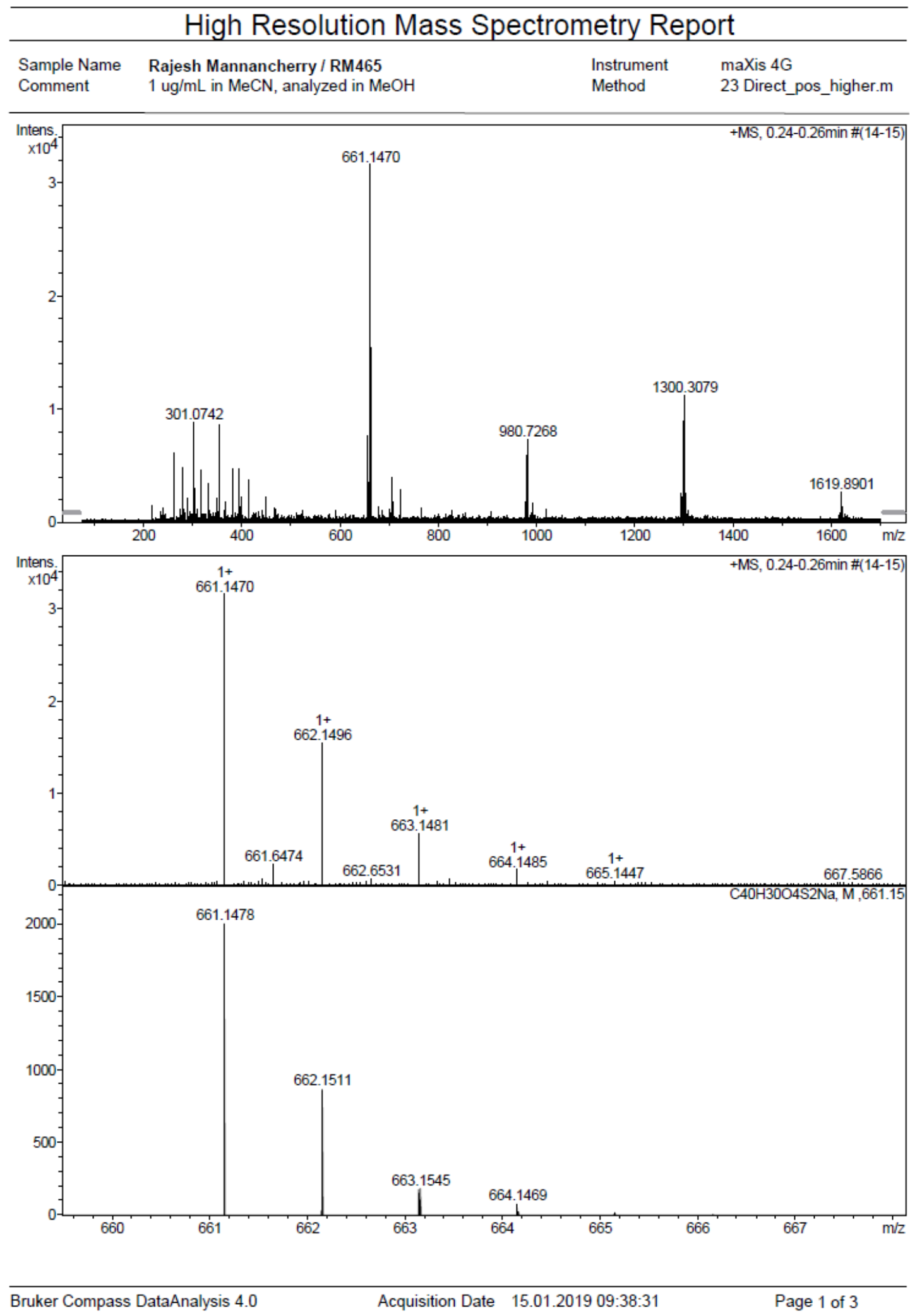




\begin{tabular}{|c|c|c|c|c|c|c|c|c|c|c|}
\hline \multicolumn{11}{|c|}{ High Resolution Mass Spectrometry Report } \\
\hline \multicolumn{11}{|c|}{ Measured $\mathrm{m} / \mathrm{z}$ vs. theoretical $\mathrm{m} / \mathrm{z}$} \\
\hline $\begin{array}{r}\text { Meas. } \mathrm{m} / \mathrm{z} \\
661.1470 \\
980.2253 \\
1299.3038\end{array}$ & $\begin{array}{l}\# \\
1 \\
1 \\
1\end{array}$ & $\begin{array}{l}\text { Formula } \\
\mathrm{C} 40 \mathrm{H} 30 \mathrm{NaO} 4 \mathrm{~S} 2 \\
\mathrm{C} 120 \mathrm{H} 90 \mathrm{Na} 2 \mathrm{O} 12 \mathrm{~S} 6 \\
\mathrm{C} 160 \mathrm{H} 120 \mathrm{Na} 2 \mathrm{O} 16 \mathrm{~S} 8\end{array}$ & $\begin{array}{r}\text { Score } \\
100.00 \\
100.00 \\
100.00\end{array}$ & $\begin{array}{r}\mathrm{m} / \mathrm{z} \\
661.1478 \\
980.2270 \\
1299.3063\end{array}$ & $\begin{array}{r}\text { er [mDa] } \\
0.7 \\
1.8 \\
2.5\end{array}$ & $\begin{array}{r}\text { err [ppm] } \\
1.1 \\
1.8 \\
1.9\end{array}$ & $\begin{array}{r}\text { mSigma } \\
19.8 \\
38.8 \\
232.2\end{array}$ & $\begin{array}{r}\mathrm{rdb} \\
25.5 \\
75.0 \\
100.0\end{array}$ & $\begin{array}{l}\mathrm{e}^{-} \text {Conf } \\
\text { even } \\
\text { even } \\
\text { even }\end{array}$ & $\begin{array}{c}z \\
1+ \\
2+\end{array}$ \\
\hline
\end{tabular}

Mass list

\begin{tabular}{rrrr}
$\#$ & $\mathbf{m} / \mathbf{z}$ & $\mathbf{I} \%$ & $\mathbf{I}$ \\
\hline 1 & 217.1052 & 4.5 & 1423 \\
2 & 234.2065 & 3.0 & 942 \\
3 & 239.0887 & 3.0 & 942 \\
4 & 239.1480 & 3.9 & 1225 \\
5 & 261.1295 & 19.5 & 6141 \\
6 & 262.1337 & 2.9 & 906 \\
7 & 273.1658 & 3.7 & 1180 \\
8 & 279.0922 & 15.3 & 4819 \\
9 & 280.0950 & 3.8 & 1190 \\
10 & 290.2674 & 6.8 & 2138
\end{tabular}

$2932435-28-2138$

$\begin{array}{lrr}301.0742 & 27.9 & 8788\end{array}$

$\begin{array}{lll}301.1398 & 5.9 & 1864\end{array}$

$\begin{array}{lll}302.0775 & 6.4 & 2021\end{array}$

$\begin{array}{lll}305.1558 & 9.5 & 3006\end{array}$

$\begin{array}{lll}309.2041 & 3.3 & 1037\end{array}$

$\begin{array}{lll}310.2361 & 3.6 & 1134\end{array}$

$\begin{array}{lrr}315.1921 & 14.5 & 4580\end{array}$

$\begin{array}{lll}316.1957 & 3.2 & 1023\end{array}$

$\begin{array}{lrr}331.1876 & 10.9 & 3436\end{array}$

$\begin{array}{lll}333.1669 & 3.2 & 1020\end{array}$

$\begin{array}{lll}348.9901 & 3.9 & 1245\end{array}$

$\begin{array}{lll}349.1832 & 6.7 & 2116\end{array}$

$\begin{array}{lll}353.2656 & 27.4 & 8649\end{array}$

$\begin{array}{lrr}354.2691 & 5.2 & 1651\end{array}$

$\begin{array}{lll}363.1568 & 3.2 & 1009\end{array}$

$\begin{array}{lll}365.1048 & 5.6 & 1757\end{array}$

$\begin{array}{lll}381.1677 & 5.1 & 1605\end{array}$

$\begin{array}{lll}381.2973 & 15.1 & 4760\end{array}$

$\begin{array}{lll}382.2997 & 3.6 & 1146\end{array}$

$\begin{array}{lll}393.2095 & 3.5 & 1102\end{array}$

$\begin{array}{lrr}393.2973 & 14.9 & 4703\end{array}$

$\begin{array}{lll}394.3008 & 3.5 & 1096\end{array}$

$\begin{array}{lll}395.1825 & 4.5 & 1413\end{array}$

$\begin{array}{lll}399.1779 & 7.1 & 2232\end{array}$

$\begin{array}{lll}413.1930 & 4.2 & 1333\end{array}$

$\begin{array}{lrr}413.2664 & 11.9 & 3747\end{array}$

$\begin{array}{lll}414.2698 & 3.3 & 1034\end{array}$

$\begin{array}{lll}433.1012 & 2.9 & 929\end{array}$

$\begin{array}{lll}441.2971 & 3.3 & 1049\end{array}$

$\begin{array}{lll}447.3444 & 6.9 & 2192\end{array}$

$\begin{array}{lll}467.2453 & 3.8 & 1205\end{array}$

$\begin{array}{lll}469.3274 & 3.7 & 1152\end{array}$

$\begin{array}{lll}522.5961 & 3.2 & 1001\end{array}$

$\begin{array}{lll}591.4954 & 3.3 & 1027\end{array}$

$\begin{array}{lll}656.1913 & 24.2 & 7636\end{array}$

$\begin{array}{lll}657.1956 & 11.0 & 3474\end{array}$

$\begin{array}{lll}658.1926 & 4.4 & 1382\end{array}$

$\begin{array}{lll}661.1470 & 100.0 & 31553\end{array}$

$\begin{array}{lll}661.6474 & 7.3 & 2298\end{array}$

$\begin{array}{lll}662.1496 & 48.8 & 15396\end{array}$

$\begin{array}{lll}663.1481 & 17.7 & 5586\end{array}$

$\begin{array}{lrr}664.1485 & 5.5 & 1733\end{array}$

$\begin{array}{lll}677.1218 & 4.2 & 1326\end{array}$

$\begin{array}{lll}685.4337 & 3.1 & 980\end{array}$

$\begin{array}{lll}699.5938 & 2.9 & 924\end{array}$

$\begin{array}{lll}700.6244 & 3.7 & 1153\end{array}$

$\begin{array}{lll}705.5809 & 12.4 & 3901\end{array}$

$\begin{array}{lll}706.5852 & 5.5 & 1733\end{array}$

$\begin{array}{llll}60 & 721.5769 & 9.3 & 2940\end{array}$

Bruker Compass DataAnalysis 4.0

Acquisition Date $\quad 15.01 .2019$ 09:38:31

Page 2 of 3 


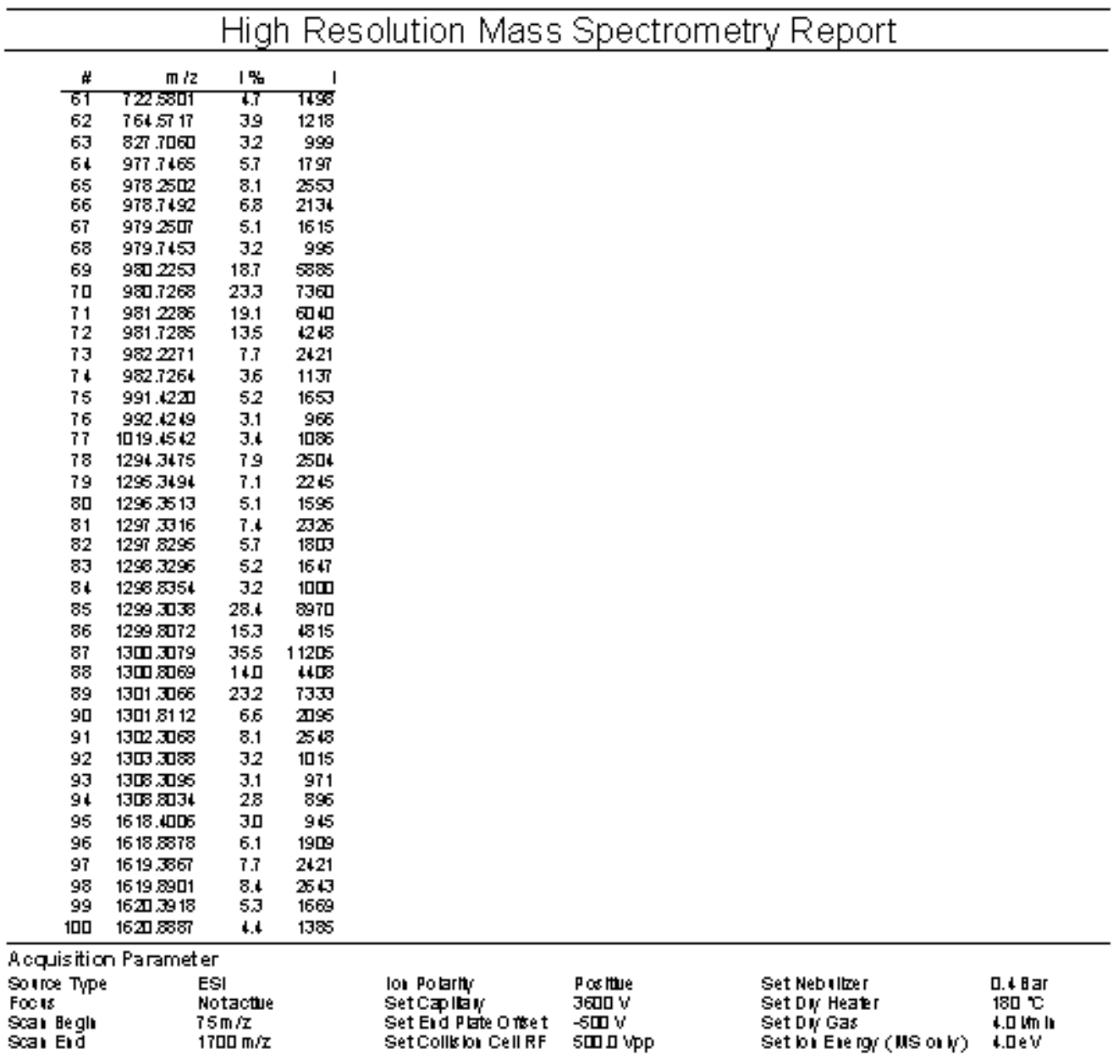


NMR $\left(\mathrm{CD}_{2} \mathrm{Cl}_{2}, 600 / 151 \mathrm{MHz}, 25^{\circ} \mathrm{C}\right)$ and HR-ESI spectra of compound 1a

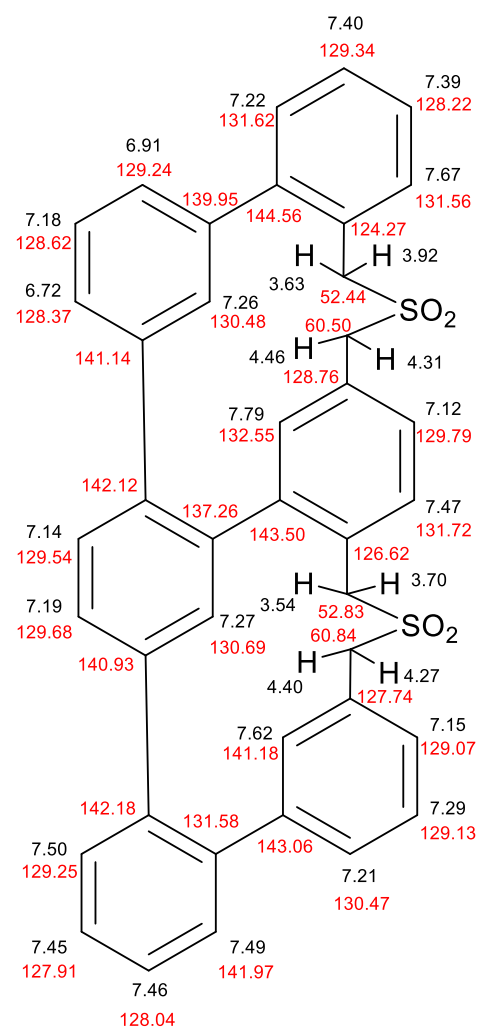

$1 \mathrm{a}$ in $\mathrm{CD}_{2} \mathrm{Cl}_{2}{ }^{1} \mathrm{H}-\mathrm{NMR}$

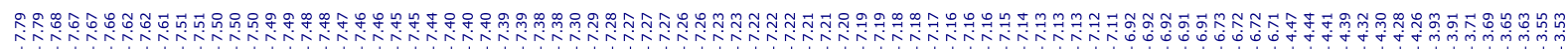

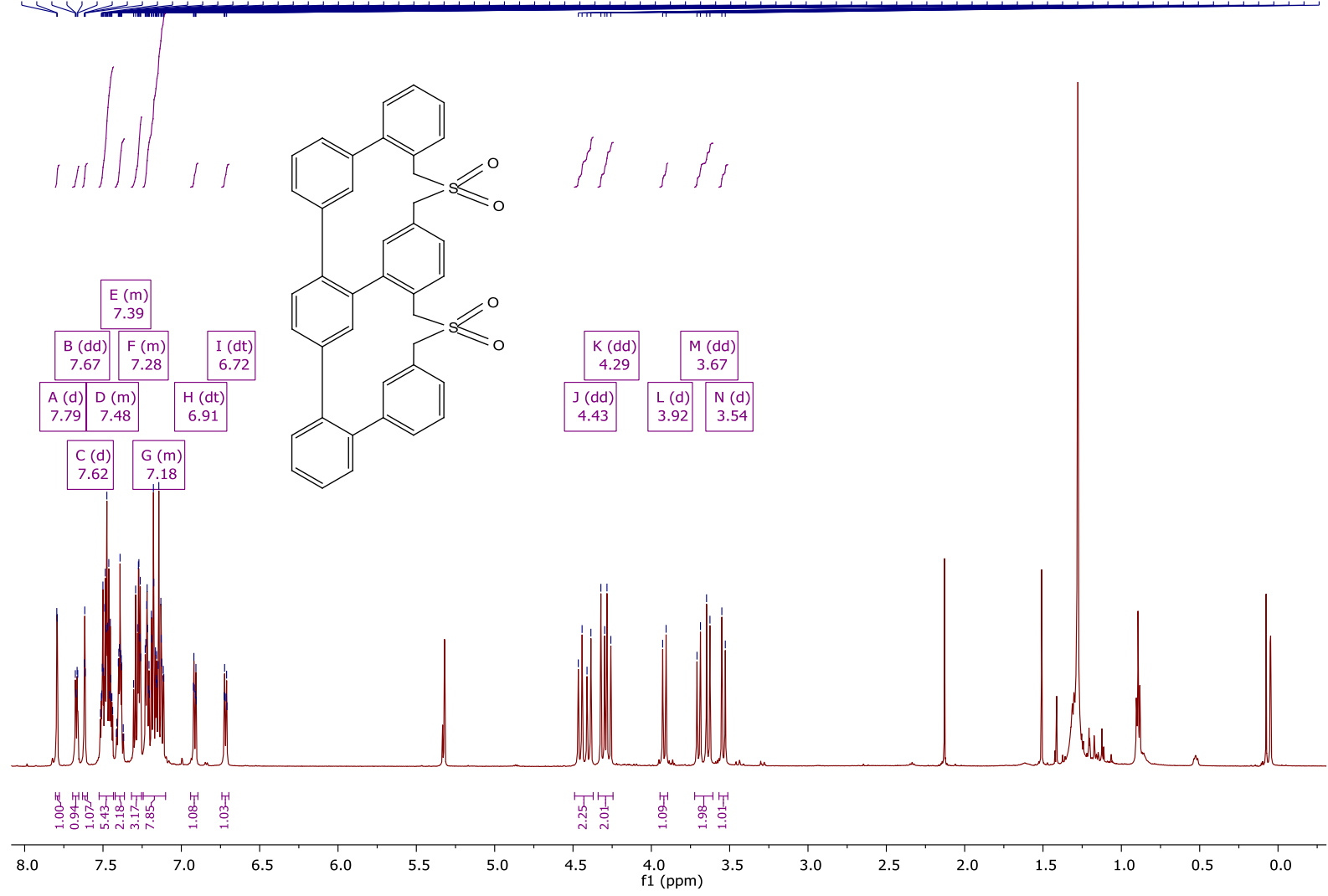



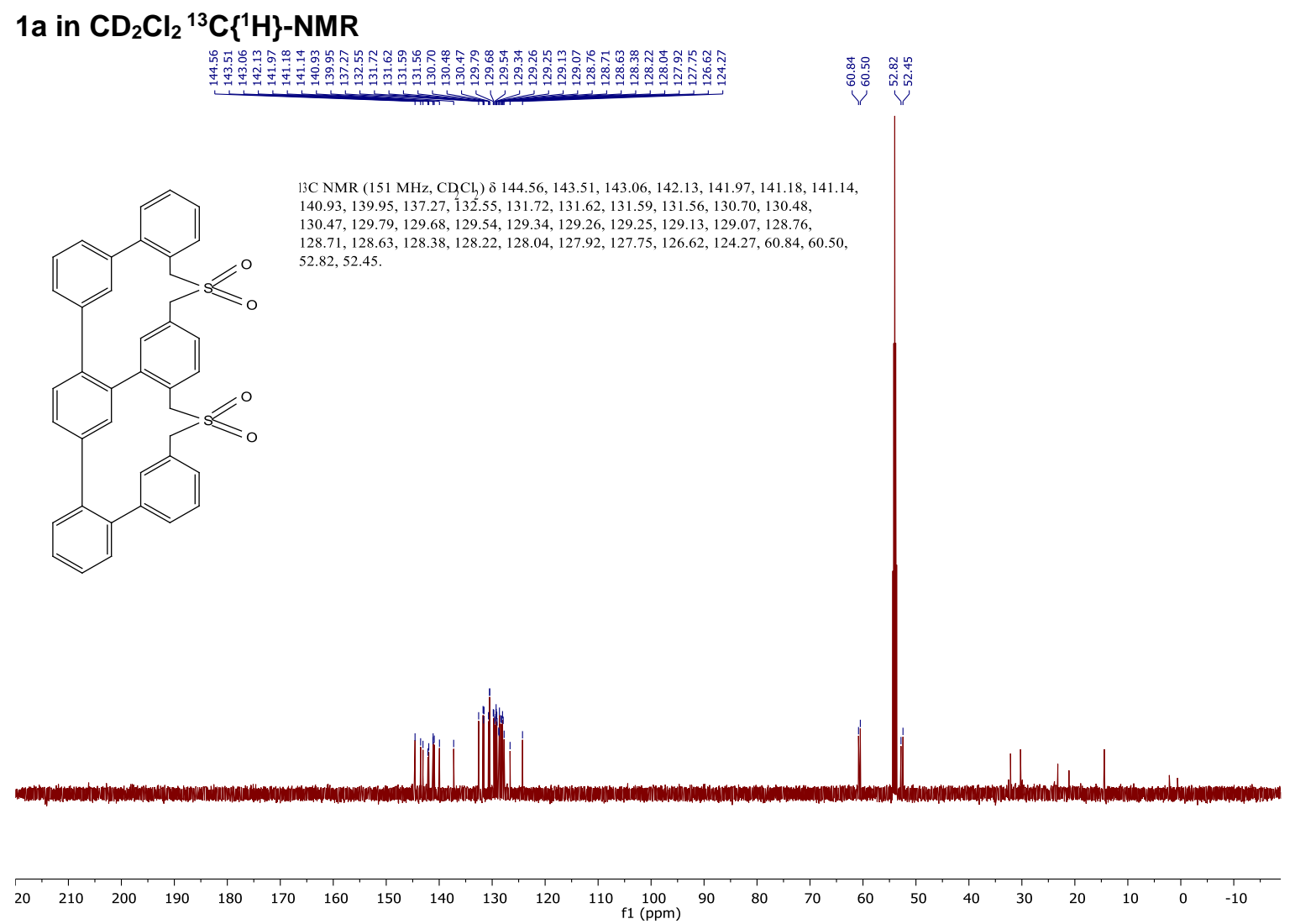

\section{$1 \mathrm{a}$ in $\mathrm{CD}_{2} \mathrm{Cl}_{2} \mathrm{gCOSY}$}

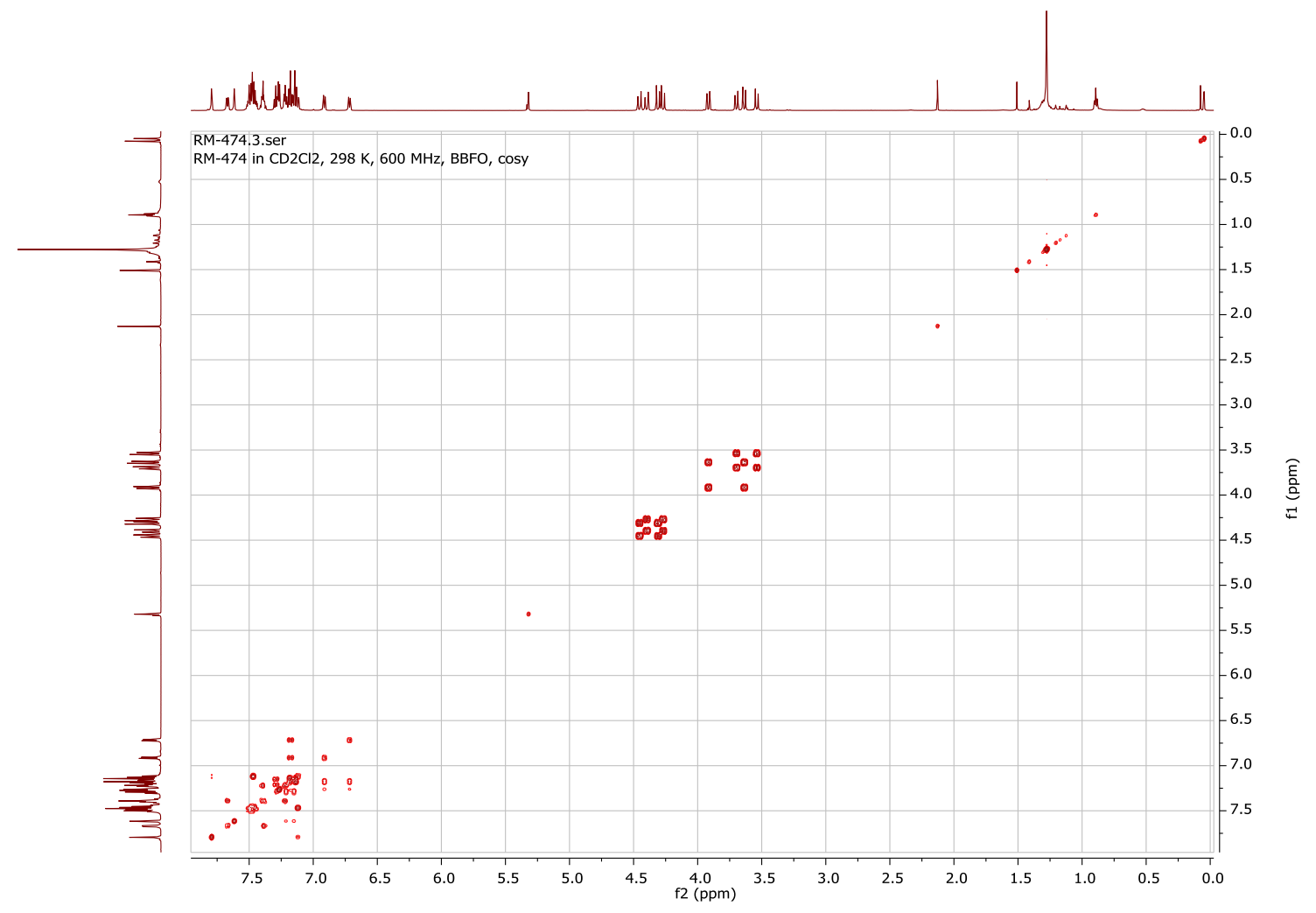




\section{1a in $\mathrm{CD}_{2} \mathrm{Cl}_{2}$ gNOESY}

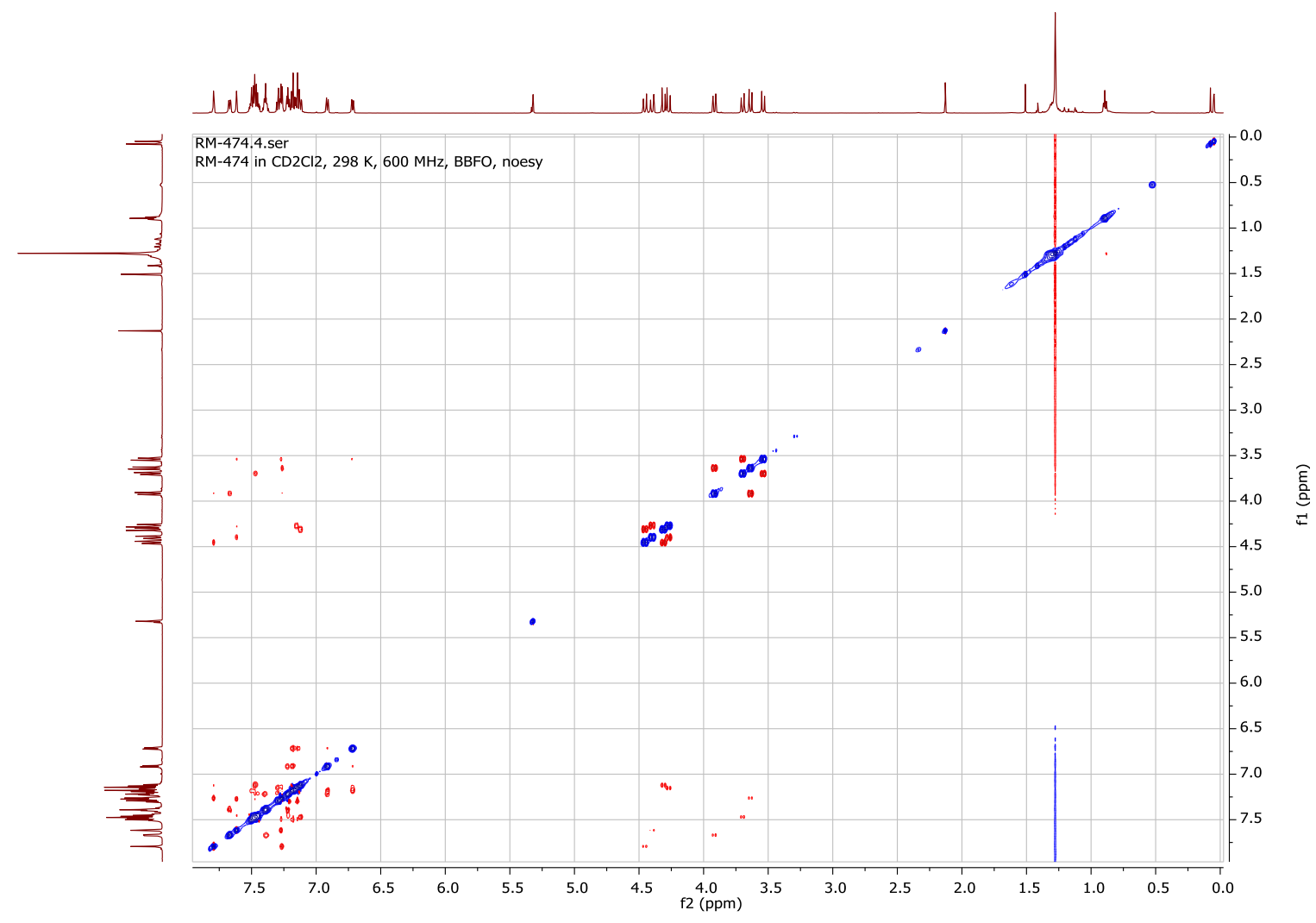

1a in $\mathrm{CD}_{2} \mathrm{Cl}_{2}$ TOCSY

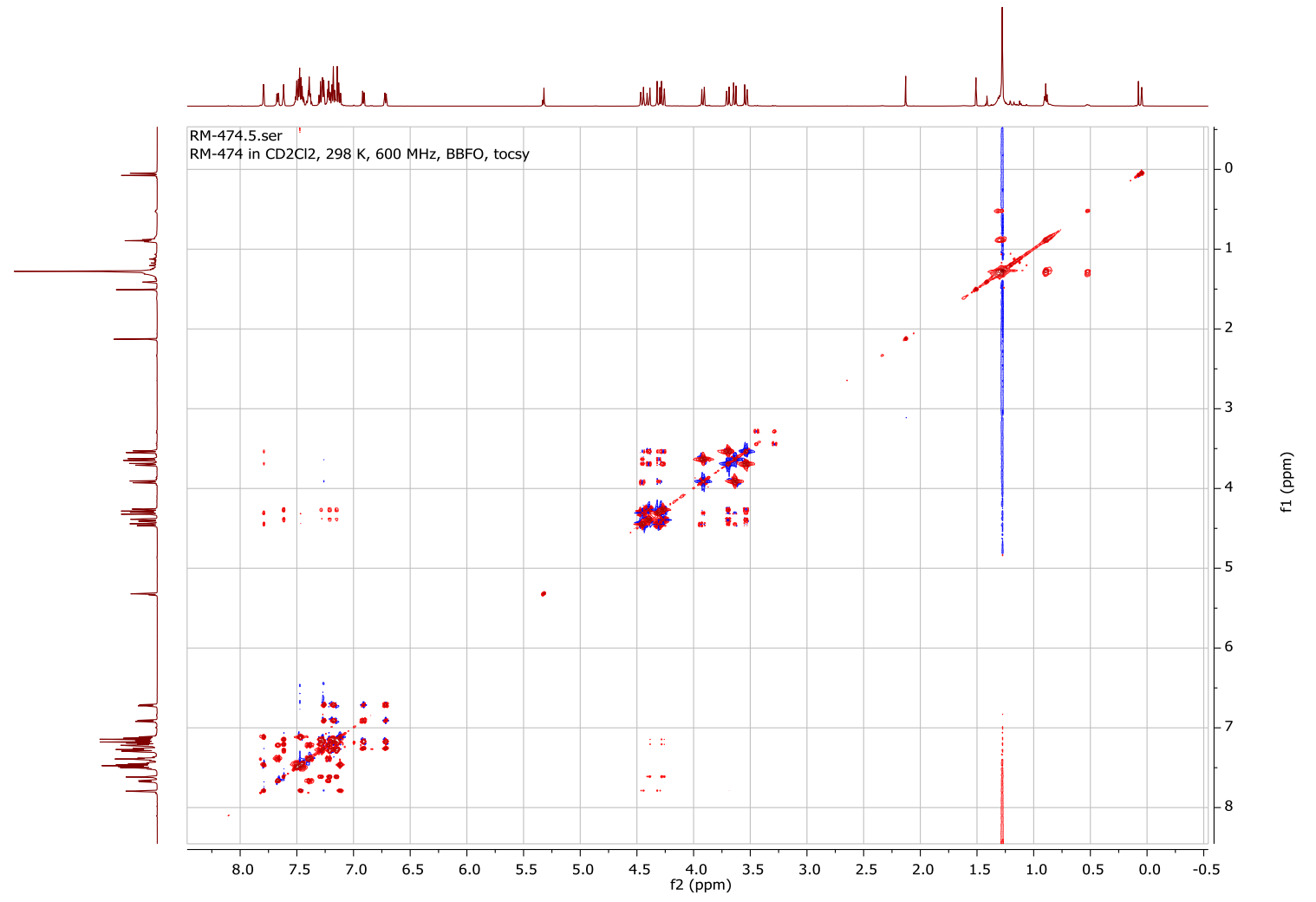


$1 a$ in $\mathrm{CD}_{2} \mathrm{Cl}_{2}$ gHSQC

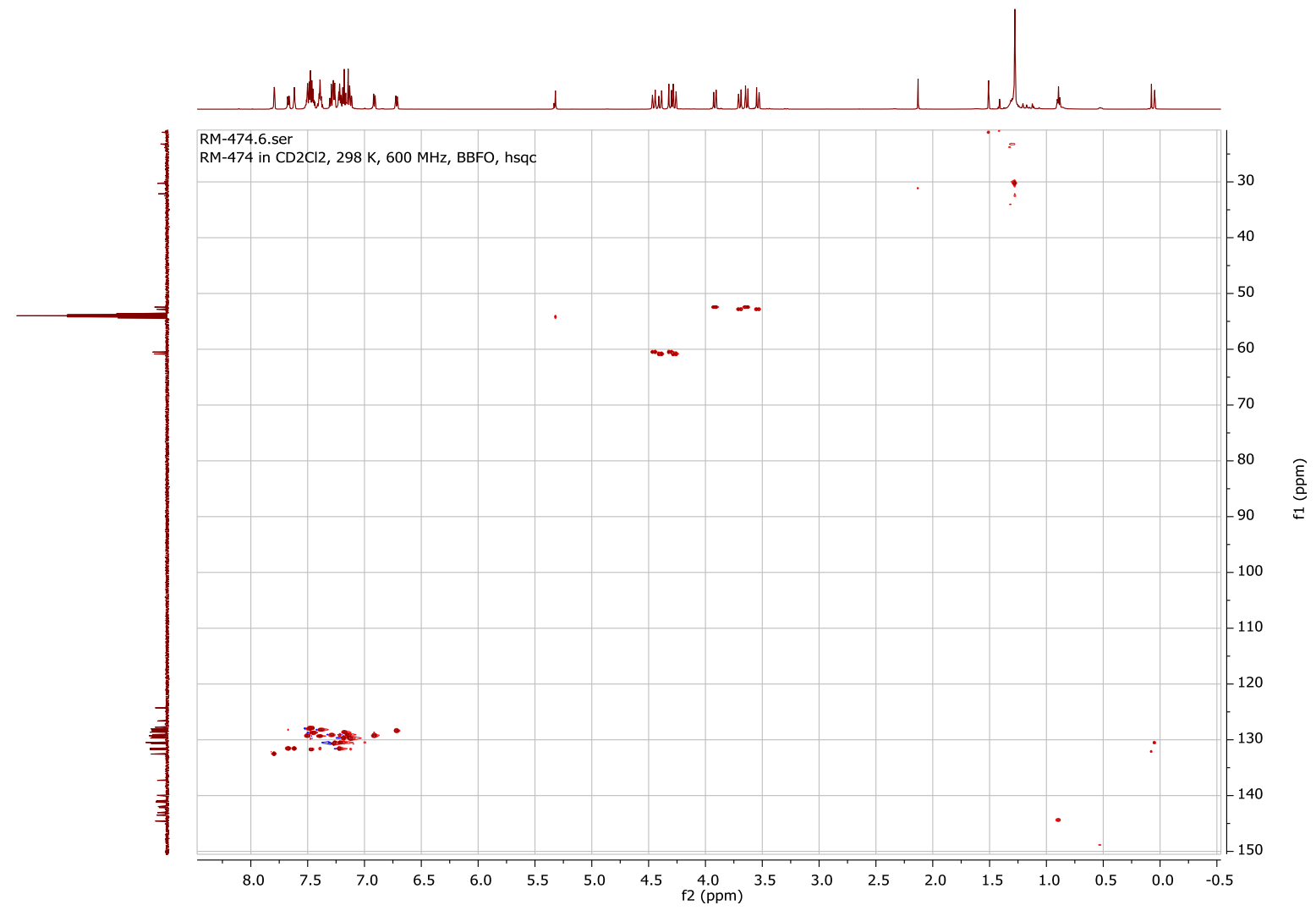

$1 a$ in $C_{2} \mathrm{Cl}_{2}$ gHMBC

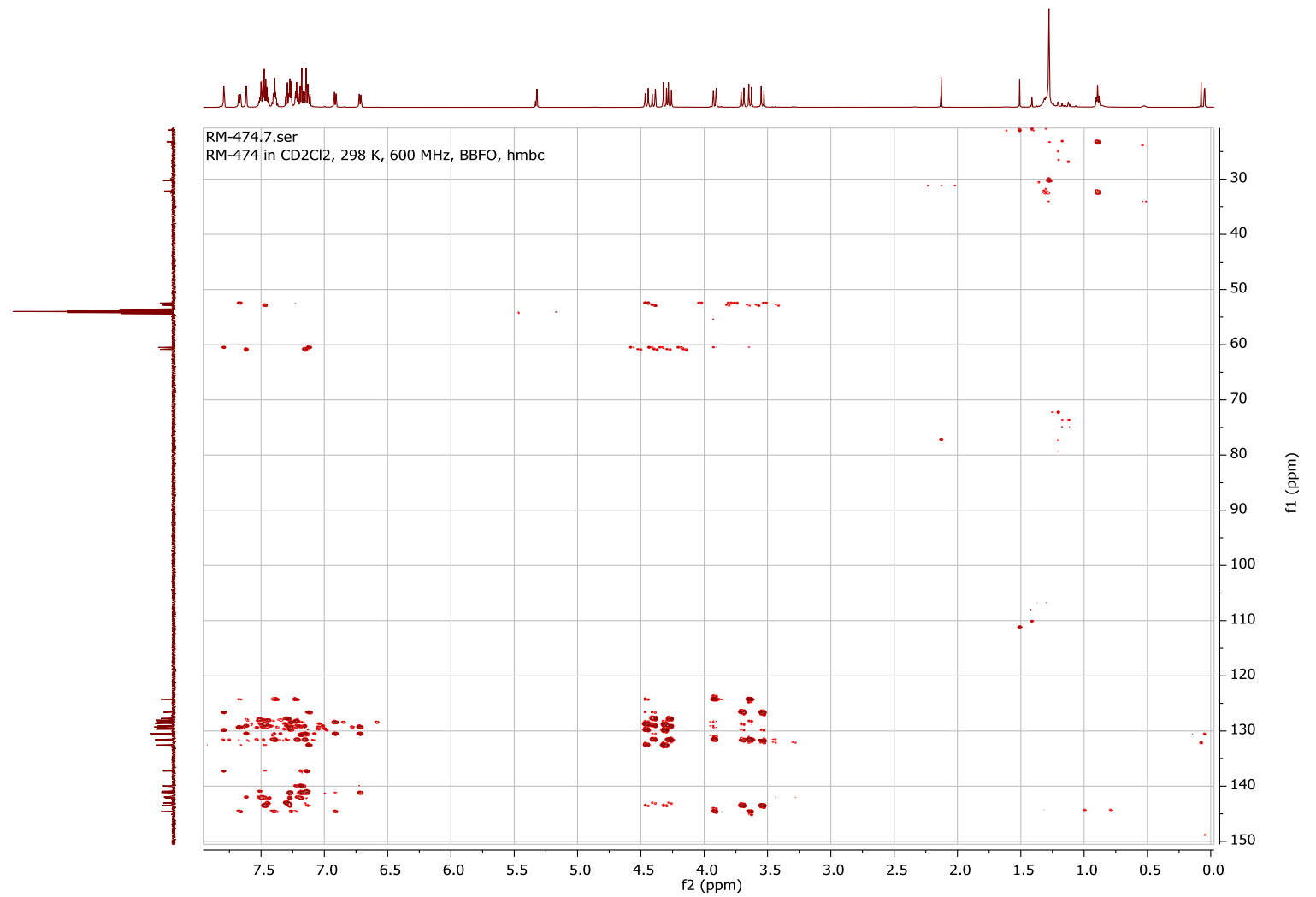




\section{HR-ESI spectra of compound 1a}

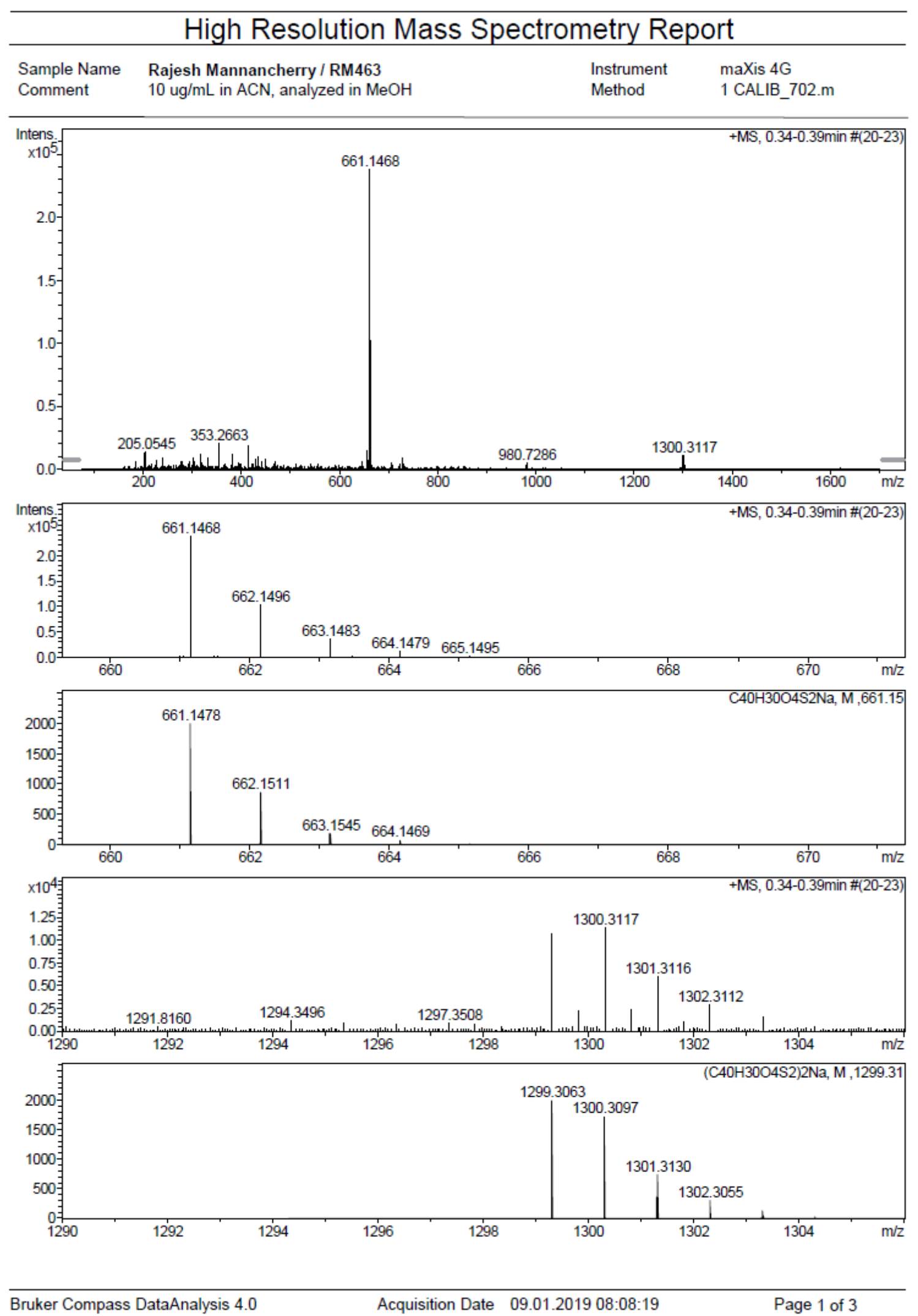




\section{High Resolution Mass Spectrometry Report}

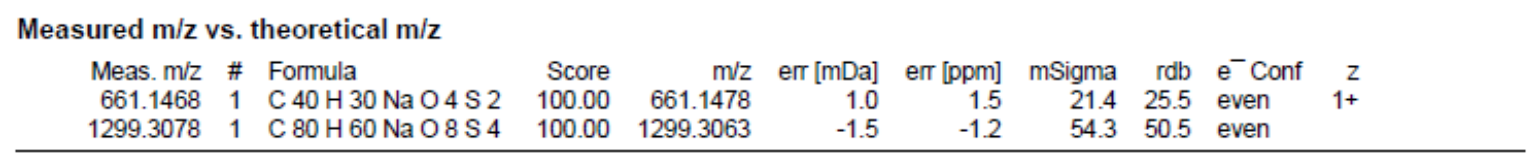

Mass list

\begin{tabular}{rrrr}
$\#$ & $\mathbf{m} / \mathbf{z}$ & $\mathbf{I} \%$ & $\mathbf{I}$ \\
\hline 1 & 185.1077 & 2.5 & 5842 \\
2 & 201.0965 & 5.5 & 13052
\end{tabular}

$205.0545 \quad 5.9 \quad 14027$

$211.0889 \quad 1.3 \quad 3087$

$217.1003 \quad 1.9 \quad 4557$

$225.1055 \quad 1.2 \quad 2935$

$226.9473 \quad 3.1 \quad 7305$

$237.1052 \quad 1.1 \quad 2542$

$\begin{array}{lll}239.0856 & 3.6 & 8595\end{array}$

$239.1221 \quad 1.2 \quad 2926$

$251.1591 \quad 1.2 \quad 2838$

$261.1279 \quad 1.4 \quad 3237$

$265.1391 \quad 1.3 \quad 3175$

$\begin{array}{lll}265.1750 & 1.2 & 2788\end{array}$

$267.1546 \quad 1.4 \quad 3408$

$273.1656 \quad 1.3 \quad 3200$

$275.1962 \quad 1.0 \quad 2487$

$277.2120 \quad 2.4 \quad 5816$

$279.2279 \quad 2.4 \quad 5656$

$281.1705 \quad 1.1 \quad 2562$

$291.1918 \quad 1.8 \quad 4181$

$293.2074 \quad 1.8 \quad 4187$

$293.2439 \quad 2.4 \quad 5751$

$2991948 \quad 1.3160$

$\begin{array}{lll}301.0743 & 1.0 & 2425\end{array}$

$301.1398 \quad 3.6 \quad 8517$

$301.2104 \quad 1.8 \quad 4332$

$303.1769 \quad 2.7 \quad 6448$

$304.2604 \quad 1.5 \quad 3490$

$305.2073-16-3765$

$305.2440 \quad 1.9 \quad 4421$

$307.1874 \quad 1.0 \quad 2447$

$307.2231 \quad 1.0 \quad 2475$

$309.2030 \quad 1.2 \quad 2866$

$\begin{array}{lll}315.1923 & 5.2 & 12369\end{array}$

$319.2237 \quad 2.0 \quad 4775$

$321.2026 \quad 1.4 \quad 3254$

$321.2397 \quad 1.2 \quad 2843$

$\begin{array}{lll}331.1876 & 3.6 & 8539\end{array}$

$331.2087 \quad 1.3 \quad 3144$

$\begin{array}{lll}335.2188 & 1.1 & 2529\end{array}$

$353.2663 \quad 8.6 \quad 20545$

$354.2701 \quad 1.6 \quad 3794$

$363.1562 \quad 1.4 \quad 3264$

$365.1054 \quad 1.6 \quad 3706$

$365.2683 \quad 1.2 \quad 2876$

$\begin{array}{lll}379.1949 & 1.2 & 2927\end{array}$

$\begin{array}{lll}381.1673 & 1.5 & 3488\end{array}$

$\begin{array}{lll}381.2977 & 5.2 & 12265\end{array}$

$382.3012 \quad 1.3 \quad 3188$

$\begin{array}{lll}393.2978 & 2.0 & 4664\end{array}$

$395.1818 \quad 1.6 \quad 3785$

$\begin{array}{lll}399.1777 & 1.8 & 4389\end{array}$

$413.1933 \quad 1.5 \quad 3462$

$\begin{array}{lll}413.2665 & 7.9 & 18752\end{array}$

$\begin{array}{lll}414.2700 & 1.9 & 4560\end{array}$

$421.3287 \quad 1.5 \quad 3608$

$\begin{array}{lll}423.2194 & 1.9 & 4466\end{array}$

$\begin{array}{lll}427.2094 & 3.5 & 8286\end{array}$

$433.1026 \quad 4.1 \quad 9731$

$\begin{array}{llll}61 & 433.3797 & 1.0 & 2472\end{array}$

Bruker Compass DataAnalysis 4.0

Acquisition Date $\quad 09.01 .2019$ 08:08:19

Page 2 of 3 


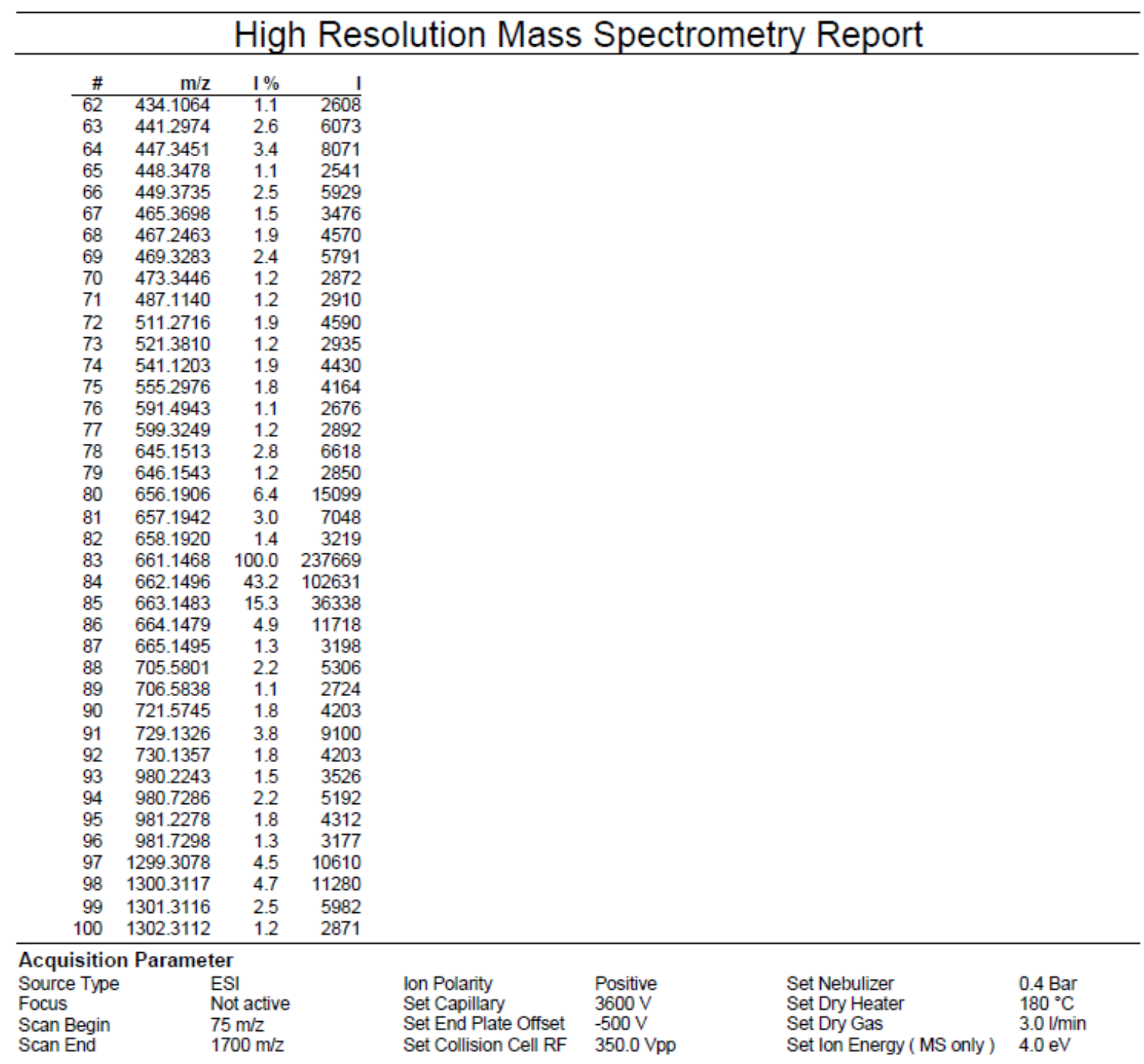




\section{NMR studies of Sulfone Geländer System 1 and 2}

a)

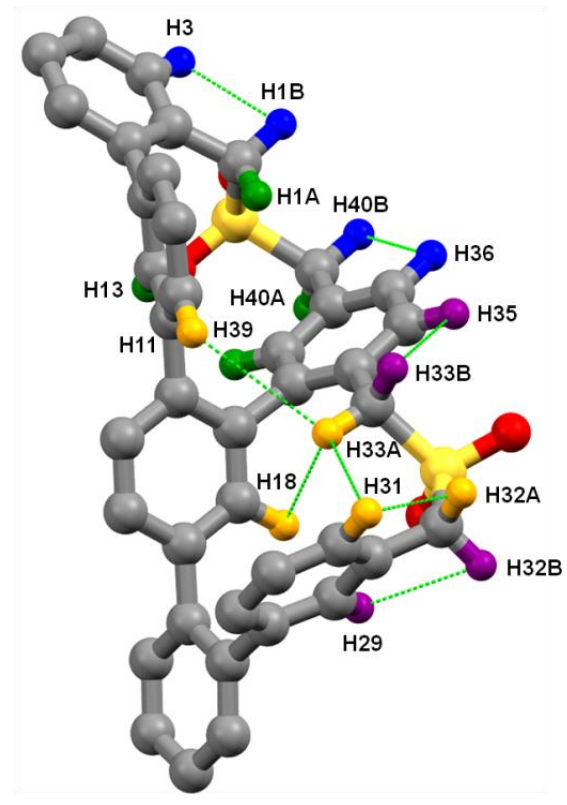

b)

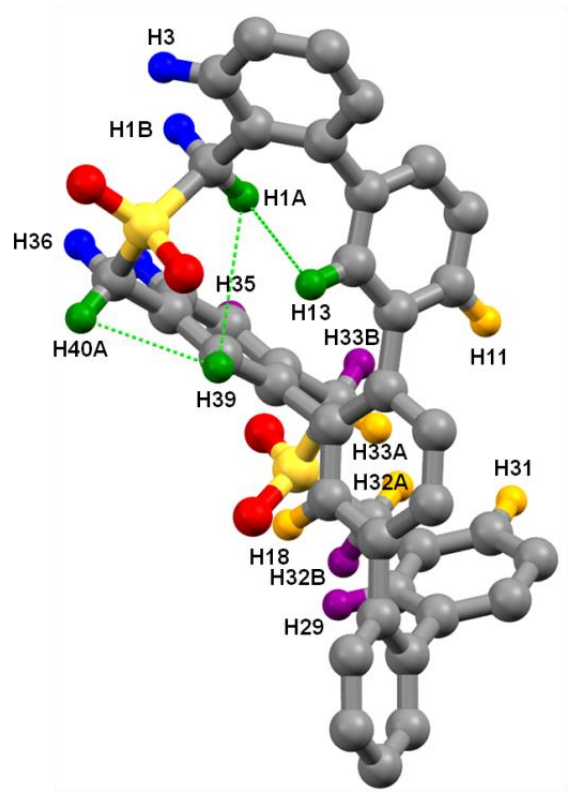

c)

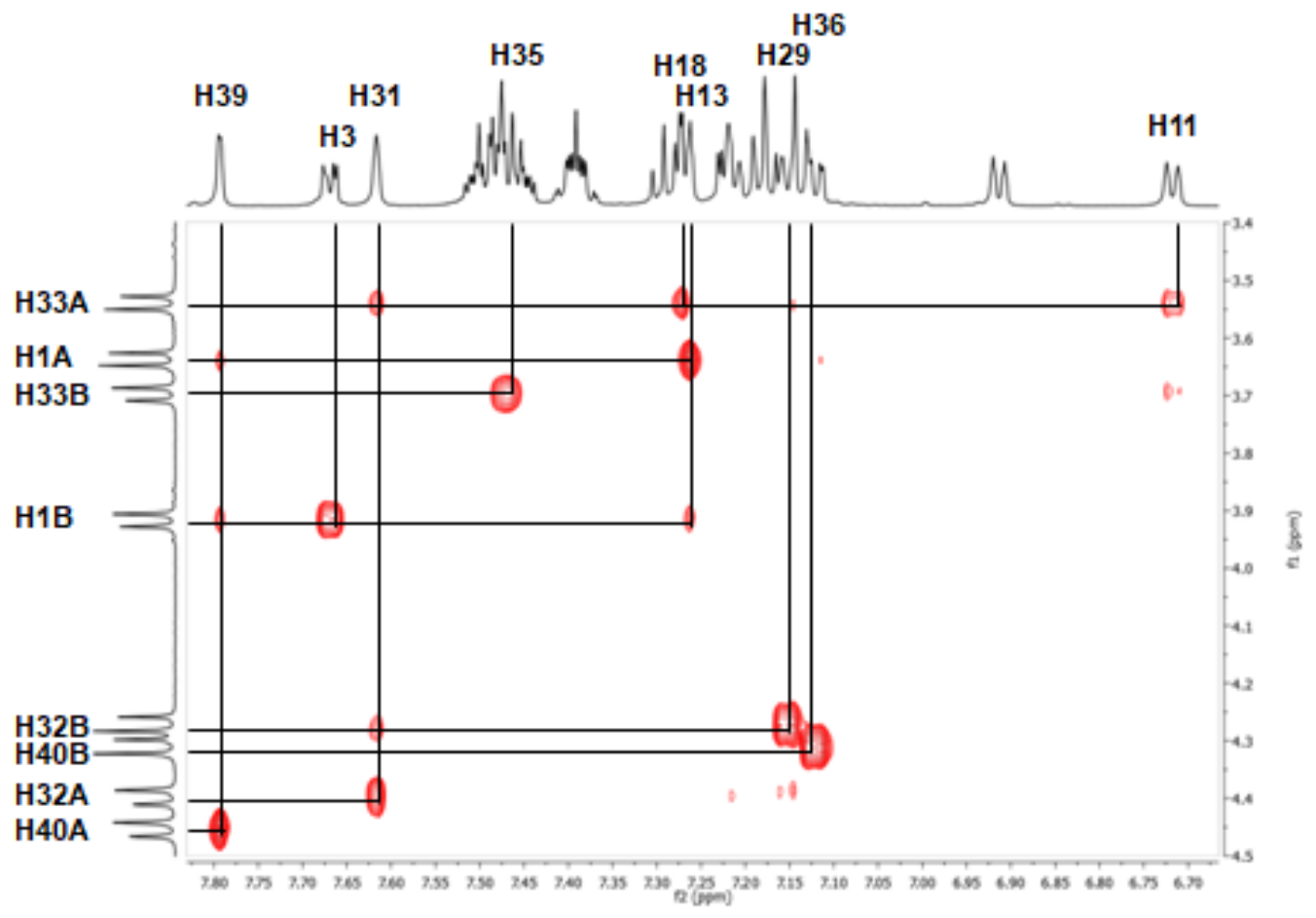

Figure S2: NOE relationships of the sulfone Geländer 1 from different viewpoints - a) front view, b) back view and c) gNOESY spectrum. Five important hydrogen groups colored in green, blue, purple and orange. The dashed green lines visualize the NOE contacts and explain the helical configuration of the structure. 
${ }^{1} \mathrm{H},{ }^{1} \mathrm{H}$-NOESY experiment particularly showed four important areas for $\mathbf{1}$ and $\mathbf{2}$ of intense NOE correlations (Figure S2 and S3 highlighted in blue, green, orange and purple) which lashes the dimethyl sulfone bridges in a helical conformation for helix 1 and 2 (Figure S2 and S3; Nuclear Overhauser effect (NOE) relationships highlighted in green dashed lines).

The helical configuration of the bottom dimethyl sulfone bridge in $\mathbf{1}$ can be explained by the NOE relationships of the protons colored in orange and purple. The a proton H33A has an NOE to protons $\mathrm{H} 18, \mathrm{H} 31$ and $\mathrm{H} 39$ and the a hydrogen $\mathrm{H} 32 \mathrm{~A}$ has only an NOE to proton $\mathrm{H} 31$ (orange area). The $\beta$ protons $\mathrm{H} 33 \mathrm{~B}$ and $\mathrm{H} 32 \mathrm{~B}$ in purple show a dipolar coupling to protons $\mathrm{H} 35$ respectively to $\mathrm{H} 29$ only. The top sulfone bridge is explained by the green and blue colored hydrogens. The a protons $\mathrm{H} 1 \mathrm{~A}$ and $\mathrm{H} 40 \mathrm{~A}$ in green show an NOE with the same hydrogen atom $\mathrm{H} 39$ but only $\mathrm{H} 1 \mathrm{~A}$ additionally interacts with $\mathrm{H} 13$. The $\beta$ protons $\mathrm{H} 1 \mathrm{~B}$ and $\mathrm{H} 40 \mathrm{~B}$ in blue are coupling only with the protons $\mathrm{H} 3$ respectively to $\mathrm{H} 36$. These NOE correlations tighten both sulfone bridges in a helical arrangement.

The observed NOE relationships by NMR spectroscopy are in good agreement with the DFT geometry optimized structure of $\mathbf{1}$.

a)

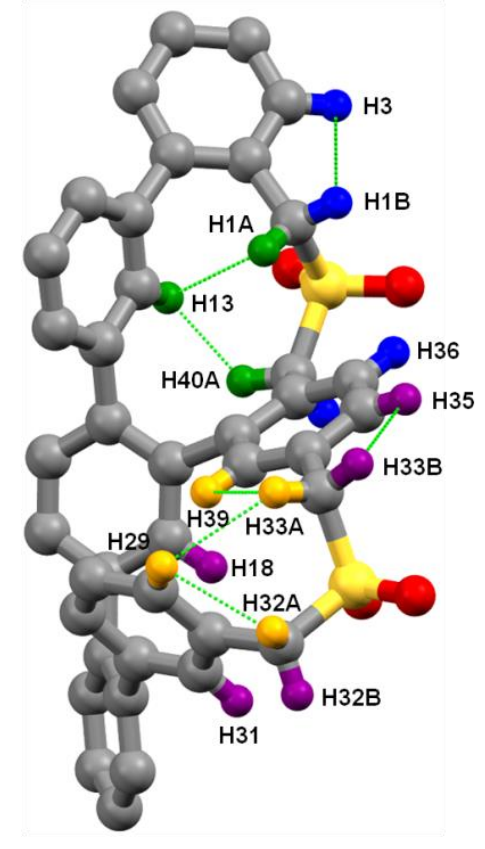

b)

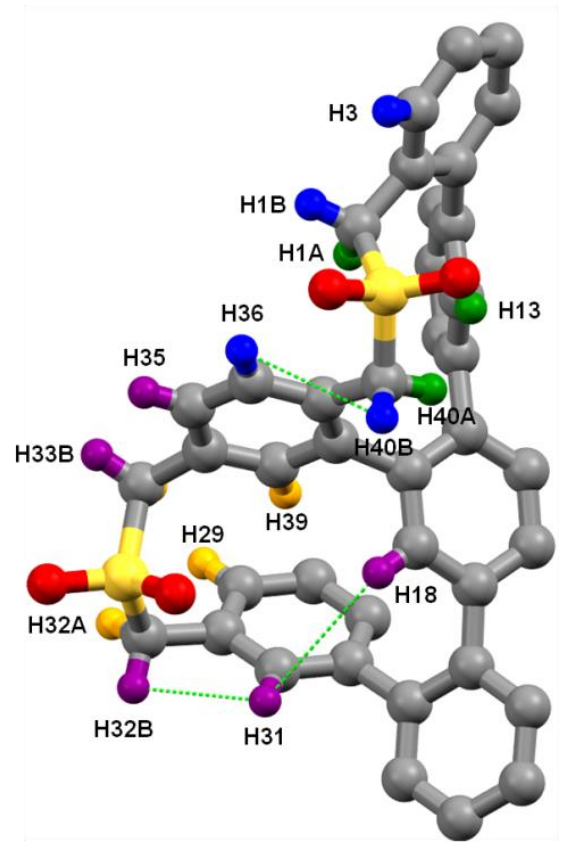


c)

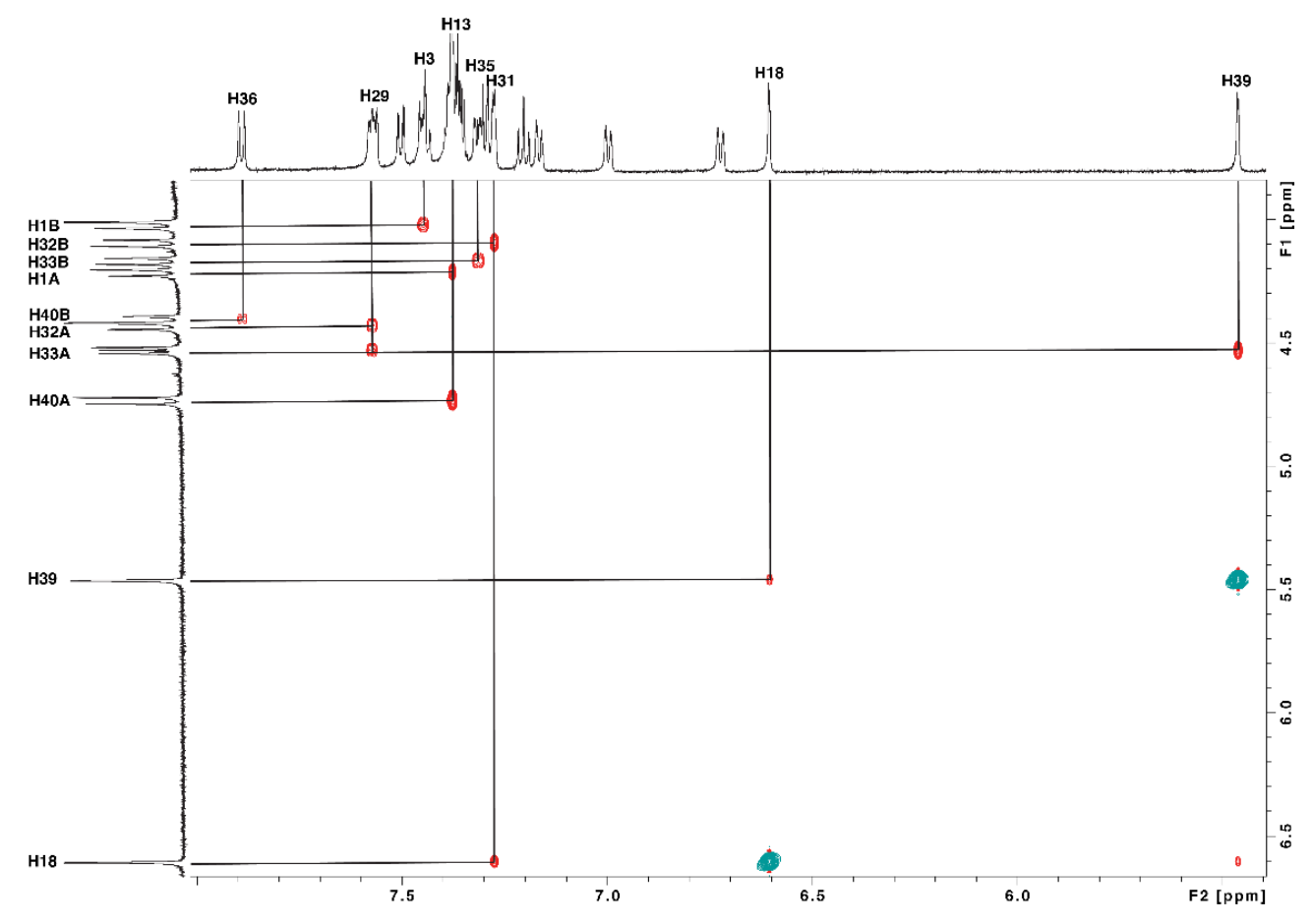

Figure S3: NOE relationships of the sulfone Geländer 2 from different viewpoints - a) front view, b) back view and c) gNOESY spectrum. Five important hydrogen groups colored in green, blue, purple and orange. The dashed green lines visualize the NOE contacts and explain the helical configuration of the structure.

The helical configuration of the bottom dimethyl sulfone bridge in $\mathbf{2}$ is explained by the NOE relationships of the protons colored in green, blue, orange and purple. The a protons $\mathrm{H} 32 \mathrm{~A}$ and $\mathrm{H} 33 \mathrm{~A}$ in orange have an NOE with $\mathrm{H} 29$ and in addition $\mathrm{H} 33 \mathrm{~A}$ correlates with $\mathrm{H} 39$ too. The $\beta$ hydrogen, H32B in purple, shows dipolar coupling to H31 and H31 shows NOE to backbone proton $\mathrm{H} 18$ of the central benzene core. The other $\beta$ proton $\mathrm{H} 33 \mathrm{~B}$ interacts only with the central benzene unit hydrogen $\mathrm{H} 35$ of the banister system. The top sulfone bridge is described by the green and blue colored hydrogens. Each of the a proton $\mathrm{H} 1 \mathrm{~A}$ and $\mathrm{H} 40 \mathrm{~A}$ (green) have an NOE contact to protons $\mathrm{H} 13$. In addition the $\beta$ hydrogens $\mathrm{H} 1 \mathrm{~B}$ and $\mathrm{H} 40 \mathrm{~B}$ in blue show only an NOE to proton $\mathrm{H} 3$ respectively to proton $\mathrm{H} 36$. These through space relations lash the structure into a helical conformation.

Overall, NMR spectroscopy confirmed the structural arrangement of macrocycle 2 to be the same as previously elucidated by $\mathrm{X}$-ray crystallography. 


\section{Experimental UV-Vis and CD: [12:14] Sulfon Geländer 2a}

Table S1: $\Delta \varepsilon$ and $\varepsilon$ values of compound 2a measured in DCM.

\begin{tabular}{|c|c|c|c|}
\hline $\begin{array}{c}\lambda \\
{[\mathrm{nm}]}\end{array}$ & $\begin{array}{c}\Delta \varepsilon \text { for enantiomer } P-\mathbf{2 a} \\
\qquad\left[\mathrm{M}^{-1} \mathrm{~cm}^{-1}\right]\end{array}$ & $\begin{array}{c}\Delta \varepsilon \text { for enantiomer } M-2 b \\
\qquad\left[\mathrm{M}^{-1} \mathrm{~cm}^{-1}\right]\end{array}$ & $\begin{array}{c}\varepsilon \text { for racemate } \\
\qquad\left[\mathrm{M}^{-1} \mathrm{~cm}^{-1}\right]\end{array}$ \\
\hline 350 & 0.024332827 & -1.277380687 & $2.0 \mathrm{E}+02$ \\
\hline 349 & -0.040917425 & -1.296537817 & $2.0 \mathrm{E}+02$ \\
\hline 348 & -0.12177062 & -1.292147079 & $2.1 \mathrm{E}+02$ \\
\hline 347 & -0.206858381 & -1.248370932 & $2.1 \mathrm{E}+02$ \\
\hline 346 & -0.285482205 & -1.156472291 & $2.2 E+02$ \\
\hline 345 & -0.350078808 & -1.021980591 & $2.2 E+02$ \\
\hline 344 & -0.397583467 & -0.865566078 & $2.3 \mathrm{E}+02$ \\
\hline 343 & -0.430003442 & -0.717483353 & $2.3 E+02$ \\
\hline 342 & -0.453788218 & -0.608170368 & $2.3 E+02$ \\
\hline 341 & -0.477446158 & -0.558833906 & $2.3 \mathrm{E}+02$ \\
\hline 340 & -0.507699591 & -0.575399053 & $2.3 \mathrm{E}+02$ \\
\hline 339 & -0.545740189 & -0.647436116 & $2.5 E+02$ \\
\hline 338 & -0.58581017 & -0.751677075 & $2.4 \mathrm{E}+02$ \\
\hline 337 & -0.61734819 & -0.858490717 & $2.4 \mathrm{E}+02$ \\
\hline 336 & -0.630042155 & -0.939409616 & $2.5 \mathrm{E}+02$ \\
\hline 335 & -0.619148972 & -0.974012496 & $2.4 \mathrm{E}+02$ \\
\hline 334 & -0.588044555 & -0.954656576 & $2.6 \mathrm{E}+02$ \\
\hline 333 & -0.546586748 & -0.88803508 & 2.6E+02 \\
\hline 332 & -0.506581661 & -0.793181617 & $2.9 \mathrm{E}+02$ \\
\hline 331 & -0.477410762 & -0.696407808 & $2.8 \mathrm{E}+02$ \\
\hline 330 & -0.46422125 & -0.624225996 & $2.9 \mathrm{E}+02$ \\
\hline 329 & -0.468521889 & -0.59567944 & $3.1 \mathrm{E}+02$ \\
\hline
\end{tabular}




\begin{tabular}{|c|c|c|c|}
\hline 328 & -0.489032516 & -0.615882628 & $3.0 \mathrm{E}+02$ \\
\hline 327 & -0.520964319 & -0.672929419 & $3.2 \mathrm{E}+02$ \\
\hline 326 & -0.554208732 & -0.740031485 & $3.2 \mathrm{E}+02$ \\
\hline 325 & -0.573057212 & -0.783215122 & $3.2 \mathrm{E}+02$ \\
\hline 324 & -0.559634675 & -0.77260594 & $3.2 E+02$ \\
\hline 323 & -0.500707365 & -0.693061197 & $3.2 \mathrm{E}+02$ \\
\hline 322 & -0.39447745 & -0.54960081 & $3.2 E+02$ \\
\hline 321 & -0.253346874 & -0.36500451 & $3.3 E+02$ \\
\hline 320 & -0.100693654 & -0.170812855 & $3.5 E+02$ \\
\hline 319 & 0.036776216 & 0.003836251 & $3.8 \mathrm{E}+02$ \\
\hline 318 & 0.137304983 & 0.140328198 & $4.0 \mathrm{E}+02$ \\
\hline 317 & 0.189500967 & 0.232992854 & 4.1E+02 \\
\hline 316 & 0.193640848 & 0.286804974 & $4.3 E+02$ \\
\hline 315 & 0.159722433 & 0.313350125 & $4.5 \mathrm{E}+02$ \\
\hline 314 & 0.103862204 & 0.327188189 & 4.7E+02 \\
\hline 313 & 0.044119012 & 0.342533579 & $5.0 \mathrm{E}+02$ \\
\hline 312 & -0.003728474 & 0.36914821 & $5.3 E+02$ \\
\hline 311 & -0.029907287 & 0.407699859 & $5.6 \mathrm{E}+02$ \\
\hline 310 & -0.032886616 & 0.446982977 & $5.9 \mathrm{E}+02$ \\
\hline 309 & -0.019030623 & 0.466016588 & $6.2 E+02$ \\
\hline 308 & 0.000778568 & 0.441789361 & $6.4 \mathrm{E}+02$ \\
\hline 307 & 0.016736506 & 0.359693164 & $6.9 \mathrm{E}+02$ \\
\hline 306 & 0.025842623 & 0.221080925 & $7.4 \mathrm{E}+02$ \\
\hline 305 & 0.035107728 & 0.043127089 & $8.1 \mathrm{E}+02$ \\
\hline 304 & 0.060381504 & -0.149755644 & $8.8 E+02$ \\
\hline 303 & 0.121106499 & -0.338694819 & $9.5 E+02$ \\
\hline
\end{tabular}




\begin{tabular}{|c|c|c|c|}
\hline 302 & 0.233809643 & -0.520154877 & $1.0 \mathrm{E}+03$ \\
\hline 301 & 0.408208228 & -0.707084541 & $1.1 \mathrm{E}+03$ \\
\hline 300 & 0.648226956 & -0.920906754 & $1.3 \mathrm{E}+03$ \\
\hline 299 & 0.956601658 & -1.179568541 & $1.4 \mathrm{E}+03$ \\
\hline 298 & 1.338901338 & -1.489342386 & 1.6E+03 \\
\hline 297 & 1.802994277 & -1.845472779 & $1.8 \mathrm{E}+03$ \\
\hline 296 & 2.353626516 & -2.240453972 & $2.0 \mathrm{E}+03$ \\
\hline 295 & 2.98577327 & -2.673178146 & $2.3 E+03$ \\
\hline 294 & 3.681913416 & -3.151218602 & $2.6 \mathrm{E}+03$ \\
\hline 293 & 4.415101592 & -3.683472618 & $3.0 \mathrm{E}+03$ \\
\hline 292 & 5.155796713 & -4.267276799 & $3.4 \mathrm{E}+03$ \\
\hline 291 & 5.877643354 & -4.879046607 & $3.8 \mathrm{E}+03$ \\
\hline 290 & 6.559197314 & -5.475453656 & $4.3 E+03$ \\
\hline 289 & 7.182155802 & -6.005584677 & 4.7E+03 \\
\hline 288 & 7.729041944 & -6.427828978 & $5.2 E+03$ \\
\hline 287 & 8.183160527 & -6.72307961 & $5.7 E+03$ \\
\hline 286 & 8.530854515 & -6.898728453 & $6.2 \mathrm{E}+03$ \\
\hline 285 & 8.764661208 & -6.983628932 & $6.6 \mathrm{E}+03$ \\
\hline 284 & 8.885583499 & -7.017075745 & $7.0 \mathrm{E}+03$ \\
\hline 283 & 8.903325848 & -7.036356395 & $7.5 \mathrm{E}+03$ \\
\hline 282 & 8.83453922 & -7.066001118 & $7.9 \mathrm{E}+03$ \\
\hline 281 & 8.699030742 & -7.111626199 & 8.3E+03 \\
\hline 280 & 8.514999964 & -7.160204173 & $8.8 \mathrm{E}+03$ \\
\hline 279 & 8.294968294 & -7.186645104 & $9.2 \mathrm{E}+03$ \\
\hline 278 & 8.044392648 & -7.164064163 & $9.7 \mathrm{E}+03$ \\
\hline 277 & 7.763582742 & -7.0729877 & $1.0 \mathrm{E}+04$ \\
\hline
\end{tabular}




\begin{tabular}{|c|c|c|c|}
\hline 276 & 7.451314457 & -6.906236134 & $1.1 \mathrm{E}+04$ \\
\hline 275 & 7.107691033 & -6.668769551 & $1.1 \mathrm{E}+04$ \\
\hline 274 & 6.734334794 & -6.374368117 & $1.2 \mathrm{E}+04$ \\
\hline 273 & 6.33285332 & -6.041579083 & $1.2 \mathrm{E}+04$ \\
\hline 272 & 5.904072522 & -5.689683098 & 1.3E+04 \\
\hline 271 & 5.450116172 & -5.334776322 & $1.3 \mathrm{E}+04$ \\
\hline 270 & 4.978992661 & -4.986585928 & $1.4 \mathrm{E}+04$ \\
\hline 269 & 4.508547578 & -4.64742791 & $1.4 \mathrm{E}+04$ \\
\hline 268 & 4.06678817 & -4.315063474 & $1.5 \mathrm{E}+04$ \\
\hline 267 & 3.687237596 & -3.988971523 & $1.5 E+04$ \\
\hline 266 & 3.401250995 & -3.677431733 & $1.6 E+04$ \\
\hline 265 & 3.230729769 & -3.401384551 & $1.7 \mathrm{E}+04$ \\
\hline 264 & 3.183711808 & -3.191787097 & 1.7E+04 \\
\hline 263 & 3.253619316 & -3.079712288 & $1.8 \mathrm{E}+04$ \\
\hline 262 & 3.42042394 & -3.082221282 & $1.9 \mathrm{E}+04$ \\
\hline 261 & 3.652652552 & -3.189779902 & $1.9 E+04$ \\
\hline 260 & 3.908803101 & -3.361009056 & $2.0 \mathrm{E}+04$ \\
\hline 259 & 4.138893193 & -3.528108022 & $2.1 \mathrm{E}+04$ \\
\hline 258 & 4.286495375 & -3.611117106 & $2.1 \mathrm{E}+04$ \\
\hline 257 & 4.291878548 & -3.534998104 & $2.2 E+04$ \\
\hline 256 & 4.096594726 & -3.242352965 & $2.2 \mathrm{E}+04$ \\
\hline 255 & 3.6494079 & -2.698017181 & $2.3 E+04$ \\
\hline 254 & 2.913417525 & -1.886691682 & $2.4 \mathrm{E}+04$ \\
\hline 253 & 1.873476973 & -0.808264527 & $2.5 E+04$ \\
\hline 252 & 0.542850974 & 0.524149966 & $2.6 \mathrm{E}+04$ \\
\hline 251 & -1.032902606 & 2.081982084 & $2.7 \mathrm{E}+04$ \\
\hline
\end{tabular}




\begin{tabular}{|c|c|c|c|}
\hline 250 & -2.776552192 & 3.816507171 & $2.9 \mathrm{E}+04$ \\
\hline 249 & -4.585386843 & 5.655541487 & $3.0 \mathrm{E}+04$ \\
\hline 248 & -6.343014981 & 7.505267975 & $3.2 E+04$ \\
\hline 247 & -7.934693904 & 9.258707014 & $3.3 E+04$ \\
\hline 246 & -9.263482251 & 10.80880178 & $3.5 E+04$ \\
\hline 245 & -10.26246644 & 12.06264232 & $3.6 \mathrm{E}+04$ \\
\hline 244 & -10.89971615 & 12.95126991 & $3.7 \mathrm{E}+04$ \\
\hline 243 & -11.17412524 & 13.43228256 & $3.8 \mathrm{E}+04$ \\
\hline 242 & -11.10482242 & 13.48582062 & $3.9 E+04$ \\
\hline 241 & -10.71981493 & 13.1085452 & $4.0 \mathrm{E}+04$ \\
\hline 240 & -10.05031043 & 12.31193977 & $4.0 \mathrm{E}+04$ \\
\hline 239 & -9.132796506 & 11.12840895 & $4.1 \mathrm{E}+04$ \\
\hline 238 & -8.016119928 & 9.623070766 & $4.1 \mathrm{E}+04$ \\
\hline 237 & -6.766958298 & 7.902094243 & $4.2 \mathrm{E}+04$ \\
\hline 236 & -5.468109243 & 6.108569209 & $4.2 E+04$ \\
\hline 235 & -4.208844946 & 4.402144848 & 4.3E+04 \\
\hline 234 & -3.070915896 & 2.928612981 & $4.3 E+04$ \\
\hline 233 & -2.116265454 & 1.791566089 & $4.4 \mathrm{E}+04$ \\
\hline 232 & -1.379879821 & 1.036297294 & $4.5 E+04$ \\
\hline 231 & -0.868552119 & 0.649285051 & 4.6E+04 \\
\hline 230 & -0.563485487 & 0.569410279 & $4.6 \mathrm{E}+04$ \\
\hline 229 & -0.425285423 & 0.705152616 & $4.6 \mathrm{E}+04$ \\
\hline 228 & -0.40095643 & 0.95343296 & $4.4 \mathrm{E}+04$ \\
\hline 227 & -0.433305613 & 1.217581722 & $3.5 \mathrm{E}+04$ \\
\hline 226 & -0.471911076 & 1.422475779 & $2.3 E+04$ \\
\hline 225 & -0.482948793 & 1.524846573 & $1.5 E+04$ \\
\hline
\end{tabular}




\begin{tabular}{|c|c|c|c|}
\hline 224 & -0.454345709 & 1.517259763 & $9.9 \mathrm{E}+03$ \\
\hline 223 & -0.394198704 & 1.425199002 & $7.7 \mathrm{E}+03$ \\
\hline 222 & -0.323182115 & 1.297645634 & $6.7 E+03$ \\
\hline 221 & -0.264052752 & 1.192893227 & $6.3 E+03$ \\
\hline 220 & -0.232175518 & 1.163057434 & $6.1 \mathrm{E}+03$ \\
\hline 219 & -0.230455852 & 1.24178386 & $6.0 \mathrm{E}+03$ \\
\hline 218 & -0.250667086 & 1.438014169 & $5.9 \mathrm{E}+03$ \\
\hline 217 & -0.281010485 & 1.735150406 & $5.7 \mathrm{E}+03$ \\
\hline 216 & -0.316481908 & 2.092944455 & $5.8 E+03$ \\
\hline 215 & -0.365600044 & 2.451923521 & $5.6 \mathrm{E}+03$ \\
\hline 214 & -0.447026068 & 2.743893162 & $5.4 \mathrm{E}+03$ \\
\hline 213 & -0.574843245 & 2.911686925 & $5.5 E+03$ \\
\hline 212 & -0.739928203 & 2.933823968 & $5.4 \mathrm{E}+03$ \\
\hline 211 & -0.900461799 & 2.84052801 & $5.4 \mathrm{E}+03$ \\
\hline 210 & -0.991322387 & 2.708246155 & $5.6 \mathrm{E}+03$ \\
\hline 209 & -0.949917674 & 2.631355155 & $5.3 E+03$ \\
\hline 208 & -0.743351311 & 2.686147712 & $5.2 \mathrm{E}+03$ \\
\hline 207 & -0.378910493 & 2.907440936 & $5.1 \mathrm{E}+03$ \\
\hline 206 & 0.1095753 & 3.290101041 & $5.0 \mathrm{E}+03$ \\
\hline 205 & 0.690050524 & 3.80944339 & $4.9 E+03$ \\
\hline 204 & 1.355005137 & 4.44352394 & $5.2 \mathrm{E}+03$ \\
\hline 203 & 2.122268061 & 5.182017219 & $5.3 \mathrm{E}+03$ \\
\hline 202 & 3.013102092 & 6.018959542 & $5.5 \mathrm{E}+03$ \\
\hline 201 & 4.023280333 & 6.93848635 & $5.9 \mathrm{E}+03$ \\
\hline 200 & 5.108115073 & 7.906359532 & $5.9 E+03$ \\
\hline
\end{tabular}




\section{Experimental UV-Vis and CD: [13:13] Sulfon Geländer 1a}

Table S2: $\Delta \varepsilon$ and $\varepsilon$ values of compound 1a measured in DCM.

\begin{tabular}{|c|c|c|c|}
\hline $\begin{array}{c}\lambda \\
{[\mathrm{nm}]}\end{array}$ & $\begin{array}{c}\Delta \varepsilon \text { for enantiomer } M-1 a \\
{\left[\mathrm{M}^{-1} \mathrm{~cm}^{-1}\right]}\end{array}$ & $\begin{array}{c}\Delta \varepsilon \text { for enantiomer } P-\mathbf{1} \mathbf{b} \\
{\left[\mathrm{M}^{-1} \mathrm{~cm}^{-1}\right]}\end{array}$ & $\begin{array}{c}\varepsilon \text { for racemate } \\
\qquad\left[\mathrm{M}^{-1} \mathrm{~cm}^{-1}\right]\end{array}$ \\
\hline 350 & 0.190983859 & -0.438927998 & $1.1 \mathrm{E}+02$ \\
\hline 349 & 0.001205476 & -0.574523183 & $1.2 \mathrm{E}+02$ \\
\hline 348 & -0.145091793 & -0.612747118 & $1.1 \mathrm{E}+02$ \\
\hline 347 & -0.231929268 & -0.568419219 & $1.1 \mathrm{E}+02$ \\
\hline 346 & -0.258959999 & -0.471439019 & $9.0 \mathrm{E}+01$ \\
\hline 345 & -0.234730753 & -0.352609507 & $7.5 \mathrm{E}+01$ \\
\hline 344 & -0.169244663 & -0.230797684 & 8.7E+01 \\
\hline 343 & -0.070594511 & -0.108793257 & $7.9 E+01$ \\
\hline 342 & 0.053436458 & 0.020163485 & $1.1 \mathrm{E}+02$ \\
\hline 341 & 0.191507818 & 0.159841583 & $1.1 \mathrm{E}+02$ \\
\hline 340 & 0.326339361 & 0.300644913 & $1.2 \mathrm{E}+02$ \\
\hline 339 & 0.436216561 & 0.417907981 & $1.0 \mathrm{E}+02$ \\
\hline 338 & 0.500310803 & 0.480089297 & $1.1 \mathrm{E}+02$ \\
\hline 337 & 0.505657129 & 0.462651649 & $1.1 \mathrm{E}+02$ \\
\hline 336 & 0.452725181 & 0.360715912 & $9.2 \mathrm{E}+01$ \\
\hline 335 & 0.356996533 & 0.194378418 & $9.3 \mathrm{E}+01$ \\
\hline 334 & 0.245641622 & 0.004074598 & $9.4 \mathrm{E}+01$ \\
\hline 333 & 0.150101305 & -0.162315531 & $1.0 \mathrm{E}+02$ \\
\hline 332 & 0.096968455 & -0.265495434 & $9.8 \mathrm{E}+01$ \\
\hline 331 & 0.099919133 & -0.287625672 & $1.0 \mathrm{E}+02$ \\
\hline 330 & 0.155228702 & -0.237207295 & $9.8 \mathrm{E}+01$ \\
\hline 329 & 0.242375477 & -0.144589091 & $1.0 \mathrm{E}+02$ \\
\hline
\end{tabular}




\begin{tabular}{|c|c|c|c|}
\hline 328 & 0.329828468 & -0.050579067 & $1.0 \mathrm{E}+02$ \\
\hline 327 & 0.384423138 & 0.006949933 & $1.1 \mathrm{E}+02$ \\
\hline 326 & 0.381242382 & 0.00342031 & $1.2 \mathrm{E}+02$ \\
\hline 325 & 0.310314615 & -0.068154031 & $1.3 E+02$ \\
\hline 324 & 0.17755163 & -0.199163826 & $1.4 \mathrm{E}+02$ \\
\hline 323 & -0.000119971 & -0.37153494 & $1.5 \mathrm{E}+02$ \\
\hline 322 & -0.20238382 & -0.564684025 & 1.7E+02 \\
\hline 321 & -0.411453631 & -0.760343467 & $1.8 \mathrm{E}+02$ \\
\hline 320 & -0.613278625 & -0.944017475 & $1.9 \mathrm{E}+02$ \\
\hline 319 & -0.794757219 & -1.103965585 & $2.0 \mathrm{E}+02$ \\
\hline 318 & -0.940915431 & -1.230038496 & $2.3 E+02$ \\
\hline 317 & -1.036008402 & -1.314278134 & $2.5 E+02$ \\
\hline 316 & -1.068769473 & -1.353239219 & $2.8 \mathrm{E}+02$ \\
\hline 315 & -1.037958127 & -1.350337814 & $3.1 \mathrm{E}+02$ \\
\hline 314 & -0.95336331 & -1.316213153 & 3.3E+02 \\
\hline 313 & -0.830957586 & -1.266574627 & 3.7E+02 \\
\hline 312 & -0.685719682 & -1.218462092 & $4.0 \mathrm{E}+02$ \\
\hline 311 & -0.527303345 & -1.186243234 & $4.5 \mathrm{E}+02$ \\
\hline 310 & -0.360950537 & -1.178116603 & $5.1 \mathrm{E}+02$ \\
\hline 309 & -0.191498804 & -1.193634963 & $5.7 \mathrm{E}+02$ \\
\hline 308 & -0.026453403 & -1.223276042 & $6.5 \mathrm{E}+02$ \\
\hline 307 & 0.123797334 & -1.251310224 & $7.3 \mathrm{E}+02$ \\
\hline 306 & 0.247356526 & -1.261976428 & $8.2 E+02$ \\
\hline 305 & 0.331472214 & -1.246698541 & $9.2 \mathrm{E}+02$ \\
\hline 304 & 0.362096178 & -1.208568997 & $1.0 \mathrm{E}+03$ \\
\hline 303 & 0.324311363 & -1.161506002 & $1.1 E+03$ \\
\hline
\end{tabular}




\begin{tabular}{|c|c|c|c|}
\hline 302 & 0.205566236 & -1.124318122 & $1.2 \mathrm{E}+03$ \\
\hline 301 & 0.001286211 & -1.112777676 & $1.3 E+03$ \\
\hline 300 & -0.280165036 & -1.133312254 & $1.5 \mathrm{E}+03$ \\
\hline 299 & -0.616672854 & -1.180546052 & $1.6 \mathrm{E}+03$ \\
\hline 298 & -0.977248626 & -1.238807887 & 1.7E+03 \\
\hline 297 & -1.331078635 & -1.28616754 & $1.8 \mathrm{E}+03$ \\
\hline 296 & -1.65683213 & -1.29909239 & $2.0 \mathrm{E}+03$ \\
\hline 295 & -1.947986309 & -1.256038773 & $2.1 \mathrm{E}+03$ \\
\hline 294 & -2.211887034 & -1.139544319 & $2.2 \mathrm{E}+03$ \\
\hline 293 & -2.463721761 & -0.937603369 & $2.4 \mathrm{E}+03$ \\
\hline 292 & -2.719154702 & -0.645873929 & $2.5 E+03$ \\
\hline 291 & -2.98955451 & -0.271203853 & $2.7 \mathrm{E}+03$ \\
\hline 290 & -3.281277953 & 0.165127938 & $2.8 \mathrm{E}+03$ \\
\hline 289 & -3.596649518 & 0.627532822 & $3.0 \mathrm{E}+03$ \\
\hline 288 & -3.933700507 & 1.071719758 & $3.2 E+03$ \\
\hline 287 & -4.283227838 & 1.454795144 & $3.4 \mathrm{E}+03$ \\
\hline 286 & -4.625591946 & 1.745850362 & $3.6 \mathrm{E}+03$ \\
\hline 285 & -4.931048852 & 1.93249994 & $3.9 \mathrm{E}+03$ \\
\hline 284 & -5.1658223 & 2.020935491 & 4.1E+03 \\
\hline 283 & -5.301852379 & 2.030552154 & $4.4 \mathrm{E}+03$ \\
\hline 282 & -5.32523959 & 1.986336627 & 4.7E+03 \\
\hline 281 & -5.239541826 & 1.912495751 & $4.9 \mathrm{E}+03$ \\
\hline 280 & -5.062240198 & 1.829038212 & $5.1 \mathrm{E}+03$ \\
\hline 279 & -4.817220021 & 1.750776683 & $5.3 E+03$ \\
\hline 278 & -4.527204367 & 1.686752491 & $5.5 \mathrm{E}+03$ \\
\hline 277 & -4.209033926 & 1.638637708 & $5.7 \mathrm{E}+03$ \\
\hline
\end{tabular}




\begin{tabular}{|c|c|c|c|}
\hline 276 & -3.873833041 & 1.598795639 & $5.9 \mathrm{E}+03$ \\
\hline 275 & -3.530211811 & 1.550813453 & $6.1 \mathrm{E}+03$ \\
\hline 274 & -3.188535562 & 1.475024074 & $6.3 E+03$ \\
\hline 273 & -2.863367933 & 1.358163293 & $6.5 E+03$ \\
\hline 272 & -2.572806736 & 1.202465033 & $6.8 \mathrm{E}+03$ \\
\hline 271 & -2.335886693 & 1.028490843 & $7.0 \mathrm{E}+03$ \\
\hline 270 & -2.170447078 & 0.869416975 & $7.2 \mathrm{E}+03$ \\
\hline 269 & -2.092223259 & 0.759808583 & $7.5 E+03$ \\
\hline 268 & -2.114621377 & 0.725135555 & 7.7E+03 \\
\hline 267 & -2.247195556 & 0.777104644 & $8.1 \mathrm{E}+03$ \\
\hline 266 & -2.492516968 & 0.915659666 & $8.4 \mathrm{E}+03$ \\
\hline 265 & -2.843728368 & 1.134919151 & $8.8 \mathrm{E}+03$ \\
\hline 264 & -3.285499985 & 1.4294174 & $9.3 E+03$ \\
\hline 263 & -3.799307059 & 1.798486932 & $9.9 E+03$ \\
\hline 262 & -4.369898092 & 2.248173278 & $1.1 \mathrm{E}+04$ \\
\hline 261 & -4.989018776 & 2.790473108 & $1.1 \mathrm{E}+04$ \\
\hline 260 & -5.654415777 & 3.439390029 & $1.2 \mathrm{E}+04$ \\
\hline 259 & -6.366207694 & 4.204070995 & 1.3E+04 \\
\hline 258 & -7.124109893 & 5.08074036 & $1.4 \mathrm{E}+04$ \\
\hline 257 & -7.926983849 & 6.046159912 & $1.5 \mathrm{E}+04$ \\
\hline 256 & -8.773145494 & 7.056078166 & 1.6E+04 \\
\hline 255 & -9.657732376 & 8.049657755 & $1.7 \mathrm{E}+04$ \\
\hline 254 & -10.56632317 & 8.959880939 & $1.9 \mathrm{E}+04$ \\
\hline 253 & -11.46786934 & 9.727438589 & $2.0 \mathrm{E}+04$ \\
\hline 252 & -12.31251295 & 10.313473 & $2.1 \mathrm{E}+04$ \\
\hline 251 & -13.0382518 & 10.70632012 & $2.3 E+04$ \\
\hline
\end{tabular}




\begin{tabular}{|c|c|c|c|}
\hline 250 & -13.58436454 & 10.91865534 & $2.4 \mathrm{E}+04$ \\
\hline 249 & -13.9052627 & 10.97488552 & $2.6 \mathrm{E}+04$ \\
\hline 248 & -13.97715584 & 10.89447322 & $2.7 E+04$ \\
\hline 247 & -13.79439877 & 10.67804771 & $2.9 E+04$ \\
\hline 246 & -13.35865185 & 10.30248317 & $3.1 E+04$ \\
\hline 245 & -12.66811242 & 9.726337358 & $3.3 E+04$ \\
\hline 244 & -11.71341134 & 8.902661902 & $3.5 \mathrm{E}+04$ \\
\hline 243 & -10.48297361 & 7.794397023 & $3.7 \mathrm{E}+04$ \\
\hline 242 & -8.975637 & 6.38812569 & $3.9 E+04$ \\
\hline 241 & -7.215927231 & 4.70481623 & $4.1 \mathrm{E}+04$ \\
\hline 240 & -5.266226018 & 2.805867861 & 4.3E+04 \\
\hline 239 & -3.231704654 & 0.793074734 & $4.5 E+04$ \\
\hline 238 & -1.255117636 & -1.200772734 & 4.7E+04 \\
\hline 237 & 0.499914691 & -3.031080733 & $5.0 \mathrm{E}+04$ \\
\hline 236 & 1.876370683 & -4.566600567 & $5.2 E+04$ \\
\hline 235 & 2.757129272 & -5.7124646 & $5.5 E+04$ \\
\hline 234 & 3.094725804 & -6.422600946 & $5.8 \mathrm{E}+04$ \\
\hline 233 & 2.925393854 & -6.699459281 & $6.1 \mathrm{E}+04$ \\
\hline 232 & 2.358830354 & -6.583627813 & $6.4 \mathrm{E}+04$ \\
\hline 231 & 1.545802173 & -6.140888208 & $6.7 \mathrm{E}+04$ \\
\hline 230 & 0.63765493 & -5.451113389 & $6.9 \mathrm{E}+04$ \\
\hline 229 & -0.24392909 & -4.600828608 & $6.9 E+04$ \\
\hline 228 & -1.016228893 & -3.678244674 & $6.4 \mathrm{E}+04$ \\
\hline 227 & -1.627009877 & -2.767482112 & $5.0 \mathrm{E}+04$ \\
\hline 226 & -2.041338285 & -1.940413068 & $3.2 \mathrm{E}+04$ \\
\hline 225 & -2.235997684 & -1.245114117 & $1.9 E+04$ \\
\hline
\end{tabular}




\begin{tabular}{|c|c|c|c|}
\hline 224 & -2.205015558 & -0.69562932 & $1.2 \mathrm{E}+04$ \\
\hline 223 & -1.970533858 & -0.269554255 & $8.6 \mathrm{E}+03$ \\
\hline 222 & -1.589561876 & 0.080401737 & $7.0 \mathrm{E}+03$ \\
\hline 221 & -1.149756588 & 0.401976097 & $6.7 \mathrm{E}+03$ \\
\hline 220 & -0.753094301 & 0.715963878 & $6.4 \mathrm{E}+03$ \\
\hline 219 & -0.492009054 & 1.000297095 & $6.1 \mathrm{E}+03$ \\
\hline 218 & -0.426211768 & 1.195765507 & $5.8 \mathrm{E}+03$ \\
\hline 217 & -0.568719582 & 1.23242974 & $5.3 E+03$ \\
\hline 216 & -0.886638599 & 1.064283081 & $5.1 \mathrm{E}+03$ \\
\hline 215 & -1.316467557 & 0.69500454 & $5.1 \mathrm{E}+03$ \\
\hline 214 & -1.78727869 & 0.182443102 & $5.1 \mathrm{E}+03$ \\
\hline 213 & -2.241384332 & -0.379535717 & $5.2 \mathrm{E}+03$ \\
\hline 212 & -2.643276721 & -0.892461909 & $5.0 \mathrm{E}+03$ \\
\hline 211 & -2.974231855 & -1.283362774 & $5.1 \mathrm{E}+03$ \\
\hline 210 & -3.218493015 & -1.521116189 & $4.9 \mathrm{E}+03$ \\
\hline 209 & -3.351320991 & -1.613763384 & $4.9 E+03$ \\
\hline 208 & -3.336330405 & -1.590268968 & $5.0 \mathrm{E}+03$ \\
\hline 207 & -3.131395813 & -1.476608498 & $5.0 \mathrm{E}+03$ \\
\hline 206 & -2.695174509 & -1.277517262 & $5.1 \mathrm{E}+03$ \\
\hline 205 & -1.98822607 & -0.971613412 & $4.8 \mathrm{E}+03$ \\
\hline 204 & -0.971809795 & -0.521106762 & $5.0 \mathrm{E}+03$ \\
\hline 203 & 0.38507779 & 0.108559077 & $5.1 \mathrm{E}+03$ \\
\hline 202 & 2.084291532 & 0.927382152 & $5.3 E+03$ \\
\hline 201 & 4.071794164 & 1.906632261 & $6.0 \mathrm{E}+03$ \\
\hline 200 & 6.226596003 & 2.978378988 & $6.5 E+03$ \\
\hline
\end{tabular}




\section{Determination of $\Delta G_{\mathrm{rac}(298 \mathrm{~K})}^{\neq}$by circular dichroism}

The Geländer model compounds $\mathbf{1 a}$ and $\mathbf{2 a}$ both consist as pair of helically wrapped enantiomers with the design intention to avoid intermediate conformations. The racemization reaction is thus the reversible transformation of one enantiomer into the other:

$$
M \rightleftarrows P
$$

for which for symmetry reasons the equilibrium constant is 1 at every temperature.

This reversible reaction causes the observed decay of enantiomeric excess in solution towards equilibrium. The racemization reaction in both directions is first order in the concentration of the corresponding enantiomer, $x$ with $x=M, P$. The reaction rate $r$ is given as:

$$
r=k_{e n} c_{x} \quad x=M, P
$$

where $c_{x}$ is the concentration of the $x$-th enantiomer and $k_{e n}$ is the first order rate constant of the enantiomerization reaction (e.g. $M \rightarrow P$ ). For the sake of symmetry, rate constants are the same in both directions. The evolution of the concentration of both species in solution is described by the following rate equations:

$$
\begin{aligned}
\frac{\mathrm{d} c_{M}}{\mathrm{~d} t} & =-k_{e n}\left(c_{M}-c_{P}\right) \\
\frac{\mathrm{d} c_{P}}{\mathrm{~d} t} & =k_{e n}\left(c_{M}-c_{P}\right)
\end{aligned}
$$

where $c_{M}$ and $c_{P}$ are the concentrations of the $M$ and $P$ enantiomer, respectively. The sum of equation (III) and (IV) results in:

$$
\begin{aligned}
& \frac{\mathrm{d}\left(c_{M}+c_{P}\right)}{\mathrm{d} t}=-k_{e n}\left(c_{M}-c_{P}\right)+k_{e n}\left(c_{M}-c_{P}\right)=0 \\
& \text { and consequently: } \quad c_{M}+c_{P}=\text { constant } \quad(\mathrm{V})
\end{aligned}
$$

The subtraction of equation (III) and (IV) results in:

$$
\frac{\mathrm{d}\left(c_{M}-c_{P}\right)}{\mathrm{d} t}=-k_{e n}\left(c_{M}-c_{P}\right)-k_{e n}\left(c_{M}-c_{P}\right)=-2 k_{e n}\left(c_{M}-c_{P}\right)
$$

As the enantiomeric excess $(E E)$ is defined as: $E E=\frac{\left(c_{M}-c_{P}\right)}{\left(c_{M}+c_{P}\right)}$ and thus, the equations $(\mathrm{V})$ and (VI) can be combined as:

$$
\frac{\mathrm{d}\left(\frac{\left.c_{M}-c_{P}\right)}{\left(c_{M}+c_{P}\right)}\right.}{\mathrm{d} t}=-2 k_{e n} \frac{\left(c_{M}-c_{P}\right)}{\left(c_{M}+c_{P}\right)}=\frac{\mathrm{d} E E}{\mathrm{~d} t}-2 k_{e n} E E
$$

Integrating equation $(\mathrm{VII})$ with $E E(0)=E E_{0}$ and linearizing gives:

$$
\ln E E(t)=\ln E E_{0}-2 k_{e n} t
$$


The analysis by circular dichroism of the sample enriched with one enantiomer provide the evolution of the enantiomeric excess over time expressed in the intensities $\left(I^{\lambda}\right)$ of the signals at the given wavelength $(\lambda)$ :

$$
E E_{t}=\frac{I^{\lambda}(t)}{I_{0}^{\lambda}}
$$

By fitting equation (VIII) to the experimental measurements using linear regression for each measurement, the first order rate constant $k_{e n}$ is obtained.

As discussed above, $k_{e n}$ is the first order rate constant of the enantiomerization reaction (e.g. $M$ $\rightarrow P$ ). For convenience often the racemization process as a whole is considered, which is the same first order process with the rate constant $k_{\text {rac }}$ as described in equation $(\mathrm{X})$ :

$$
\ln E E(t)=\ln E E_{0}-k_{\text {rac }} t \quad(\mathrm{X})
$$

The comparison of equations (VIII) and (X) display the relation between $k_{e n}$ and $k_{\text {rac, }}$ namely:

$$
k_{e n}=\frac{k_{r a c}}{2}
$$

The difference between both rate constants describing the same observed evolution of the enantiomeric excess in time arise from the difference in the chemical process they express. While $k_{e n}$ is the enantiomerization rate constant for the first order process from one enantiomer into the other (e.g. $M \rightarrow P$ ), $k_{\text {rac }}$ is the rate constant for the first order process for a sample enriched with one enantiomer to the racemic equilibrium. The relation between both rate constant becomes obvious by the following thought experiment: Assuming a macroscopic sample comprising an excess of the $M$ enantiomer, every $M$ enantiomer converting to a $P$ enantiomer reduces the observed CD signal twice, first by disappearing as $M$ enantiomer and second by appearing as $P$ enantiomer, compensating the optical effect of another $M$ enantiomer. It is thus apparent that $k_{\text {rac }}$ must be twice $k_{e n}$. The issue is perfectly discussed in reference 2.

Equation $(\mathrm{X})$ can be brought into a linear form:

$$
\ln \left(\frac{E E(t)}{E E_{0}}\right)=-k_{\text {rac }} t
$$

Plotting $t$ against $\ln \left(\frac{E E(t)}{E E_{0}}\right)$ gives access to $k_{r a c}$ directly from the slope, while consideration of equation $(\mathrm{XI})$ provides to $k_{e n}$. The half-live of the enantiomerization process can be determined with equation (XIII):

$$
t_{1 / 2}=\frac{\ln (2)}{k_{e n}}
$$


The obtained $k_{r a c}$ and $k_{e n}$ can be used to calculate the free Gibbs energy of the enantiomerization process $G_{e n(T)}^{\neq}$by the rearranged Eyring equation:

$$
\Delta G_{e n(298.15)}^{\neq}=-R T \ln \left(\frac{h k_{e n}}{k_{B} T}\right)=-R T \ln \left(\frac{h k_{r a c}}{2 k_{B} T}\right) \quad(\mathrm{XIV})
$$

Where $k_{r a c}$ is the obtained kinetic rate constant at $\mathrm{RT}, k_{B}$ the Boltzmann constant $\left(k_{B}=\right.$ $\left.1.380662 \times 10^{-23} \mathrm{~J} \mathrm{~K}^{-1}\right), h$ the Planck's constant $\left(h=6.626176 \times 10^{-34} \mathrm{~J} \mathrm{~s}\right), R$ the universal gas constant $\left(R=8.31446 \times 10^{-3} \mathrm{~kJ} \mathrm{~K}^{-1} \mathrm{~mol}^{-1}\right)$ and $T$ the temperature $(T=298.15 \mathrm{~K})$.

Table S3: Rate constants and half-lifes of compound 1a and $2 \mathrm{a}$ by ECD determined at $25^{\circ} \mathrm{C}$.

\begin{tabular}{|c|c|c|c|c|c|c|c|c|c|}
\hline Temperature & \multicolumn{2}{|c|}{$\begin{array}{l}\text { Rate constant [1/s] } \\
\text { of compound } \mathbf{1 a}\end{array}$} & \multicolumn{2}{|c|}{ Half-life [h] } & \multicolumn{3}{|c|}{$\begin{array}{l}\text { Rate constant }[1 / \mathrm{s}] \\
\text { of compound } 2 \mathrm{a}\end{array}$} & \multicolumn{2}{|c|}{ Half-life $[\mathrm{h}]$} \\
\hline \multirow{4}{*}{$25^{\circ} \mathrm{C}$} & \multirow{2}{*}{$\begin{array}{l}k_{\text {rac }}= \\
k_{e n}=\end{array}$} & $2.67 \times 10^{-5}$ & & & \multirow{2}{*}{$\begin{array}{l}k_{\text {rac }}= \\
k_{\text {en }}=\end{array}$} & \multicolumn{2}{|c|}{$1.61 \times 10^{-4}$} & \multirow[b]{2}{*}{$t_{e n}=$} & \multirow[b]{2}{*}{2.39} \\
\hline & & $1.34 \times 10^{-5}$ & $t_{e n}=$ & 14.41 & & 8.05 & & & \\
\hline & \multicolumn{9}{|c|}{ Racemization barrier $\left[\mathrm{kJ} \mathrm{mol}^{-1}\right]$} \\
\hline & \multicolumn{2}{|c|}{$\Delta G_{e n(298.15)}^{ \pm}=$} & \multicolumn{2}{|c|}{100.8} & \multicolumn{2}{|c|}{$\Delta G_{e n(298.15)}^{\neq}=$} & & \multicolumn{2}{|c|}{96.4} \\
\hline
\end{tabular}

Table S4: Mdeg values of compound $P-2 \mathrm{a}$ at $242 \mathrm{~nm}$ at $15^{\circ} \mathrm{C}$.

\begin{tabular}{|r|r|r|r|r|}
\hline time [s] & Intensity A (mdeg) & Intensity B (mdeg) & {$[A]_{t}$} & $\ln \left(\frac{[A]_{t}}{[A]_{0}}\right)$ \\
\hline 0 & -6.54865 & 0 & -6.54865 & 0 \\
\hline 600 & -6.14754 & -0.40111 & -6.14754 & -0.06320692 \\
\hline 1200 & -6.1736 & -0.37505 & -6.1736 & -0.05897679 \\
\hline 1800 & -5.8267 & -0.72195 & -5.8267 & -0.11680812 \\
\hline 2400 & -5.81336 & -0.73529 & -5.81336 & -0.1191002 \\
\hline 3000 & -5.31813 & -1.23052 & -5.31813 & -0.20813718 \\
\hline 3600 & -5.40382 & -1.14483 & -5.40382 & -0.19215281 \\
\hline
\end{tabular}




\begin{tabular}{|c|c|c|c|c|}
\hline 4200 & -5.18639 & -1.36226 & -5.18639 & -0.23322103 \\
\hline 4800 & -4.83687 & -1.71178 & -4.83687 & -0.3029911 \\
\hline 5400 & -4.86114 & -1.68751 & -4.86114 & -0.29798594 \\
\hline 6000 & -4.58569 & -1.96296 & -4.58569 & -0.35631834 \\
\hline 6600 & -4.53949 & -2.00916 & -4.53949 & -0.36644425 \\
\hline 7200 & -4.41672 & -2.13193 & -4.41672 & -0.39386158 \\
\hline 7800 & -4.11577 & -2.43288 & -4.11577 & -0.46443298 \\
\hline 8400 & -4.12943 & -2.41922 & -4.12943 & -0.46111954 \\
\hline 9000 & -3.8377 & -2.71095 & -3.8377 & -0.53438569 \\
\hline 9600 & -3.59629 & -2.95236 & -3.59629 & -0.59935616 \\
\hline 10200 & -3.52334 & -3.02531 & -3.52334 & -0.61984952 \\
\hline 10800 & -3.4344 & -3.11425 & -3.4344 & -0.64541668 \\
\hline 11400 & -3.22824 & -3.32041 & -3.22824 & -0.70732182 \\
\hline 12000 & -3.01518 & -3.53347 & -3.01518 & -0.77559939 \\
\hline 12600 & -3.14024 & -3.40841 & -3.14024 & -0.73495969 \\
\hline 13200 & -3.12382 & -3.42483 & -3.12382 & -0.74020231 \\
\hline 13800 & -3.01525 & -3.5334 & -3.01525 & -0.77557618 \\
\hline 14400 & -2.8742 & -3.67445 & -2.8742 & -0.82348455 \\
\hline 15000 & -2.58277 & -3.96588 & -2.58277 & -0.93039646 \\
\hline 15600 & -2.60594 & -3.94271 & -2.60594 & -0.92146547 \\
\hline 16200 & -2.53894 & -4.00971 & -2.53894 & -0.94751225 \\
\hline 16800 & -2.48829 & -4.06036 & -2.48829 & -0.96766319 \\
\hline 17400 & -2.39147 & -4.15718 & -2.39147 & -1.00735068 \\
\hline 18000 & -2.30565 & -4.243 & -2.30565 & -1.04389629 \\
\hline 18600 & -2.19712 & -4.35153 & -2.19712 & -1.09211151 \\
\hline 19200 & -2.03666 & -4.51199 & -2.03666 & -1.16794771 \\
\hline
\end{tabular}




\begin{tabular}{|l|l|l|l|l|}
\hline 19800 & -1.88088 & -4.66777 & -1.88088 & -1.24751917 \\
\hline 20400 & -1.87108 & -4.67757 & -1.87108 & -1.25274312 \\
\hline 21000 & -1.94147 & -4.60718 & -1.94147 & -1.2158135 \\
\hline 21600 & -1.71218 & -4.83647 & -1.71218 & -1.34149151 \\
\hline 22200 & -1.69499 & -4.85366 & -1.69499 & -1.35158208 \\
\hline 22800 & -1.63303 & -4.91562 & -1.63303 & -1.38882174 \\
\hline 23400 & -1.7831 & -4.76555 & -1.7831 & -1.3009055 \\
\hline 24000 & -1.46015 & -5.0885 & -1.46015 & -1.50071975 \\
\hline 24600 & -1.44227 & -5.10638 & -1.44227 & -1.51304066 \\
\hline 25200 & -1.3143 & -5.23435 & -1.3143 & -1.60595472 \\
\hline 25800 & -1.27019 & -5.27846 & -1.27019 & -1.64009243 \\
\hline 26400 & -1.33367 & -5.21498 & -1.33367 & -1.59132438 \\
\hline 27000 & -1.30486 & -5.24379 & -1.30486 & -1.61316317 \\
\hline 27600 & -1.12463 & -5.42402 & -1.12463 & -1.76180483 \\
\hline 28200 & -1.15853 & -5.39012 & -1.15853 & -1.73210696 \\
\hline 28800 & -1.09415 & -5.4545 & -1.09415 & -1.78928112 \\
\hline 29400 & -0.997174 & -5.551476 & -0.997174 & -1.88208892 \\
\hline 30000 & -0.880949 & -5.667701 & -0.880949 & -2.00601447 \\
\hline 30600 & -0.877733 & -5.670917 & -0.877733 & -2.00967175 \\
\hline 31200 & -0.983402 & -5.565248 & -0.983402 & -1.89599621 \\
\hline 31800 & -0.901271 & -5.647379 & -0.901271 & -1.98320821 \\
\hline 32400 & -0.744827 & -5.803823 & -0.744827 & -2.17386222 \\
\hline 33000 & -0.870748 & -5.677902 & -0.870748 & -2.01766159 \\
\hline 33600 & -0.706665 & -5.841985 & -0.706665 & -2.22645748 \\
\hline 34200 & -0.698714 & -5.849936 & -0.698714 & -2.2377727 \\
\hline 34800 & -0.570528 & -5.978122 & -0.570528 & -2.44045195 \\
\hline & & & & \\
\hline & & & & \\
\hline
\end{tabular}




\begin{tabular}{|l|l|l|l|l|}
\hline 35400 & -0.571913 & -5.976737 & -0.571913 & -2.43802732 \\
\hline 36000 & -0.582833 & -5.965817 & -0.582833 & -2.4191135 \\
\hline
\end{tabular}
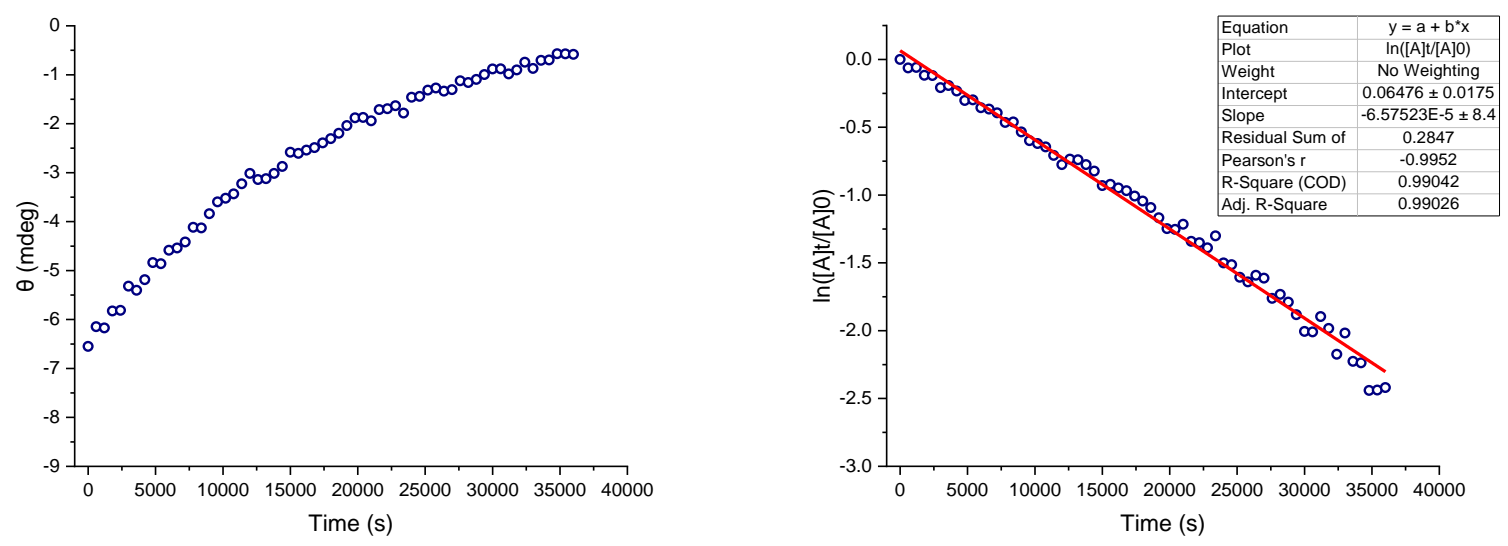

Figure S4: CD measurements of compound $P-2 \mathrm{a}$ at $15^{\circ} \mathrm{C}$ and determination of $k_{r a c}$.

Table S5: Mdeg values of compound P-2a at $242 \mathrm{~nm}$ at $20^{\circ} \mathrm{C}$.

\begin{tabular}{|c|c|c|c|c|}
\hline time $[s]$ & Intensity A (mdeg) & Intensity B (mdeg) & {$[A]_{t}$} & $\ln \left(\frac{[A]_{t}}{[A]_{0}}\right)$ \\
\hline 0 & -5.15836 & 0 & -5.15836 & 0 \\
\hline 600 & -4.53368 & -0.62468 & -4.53368 & -0.129084728 \\
\hline 1200 & -4.56011 & -0.59825 & -4.56011 & -0.123271953 \\
\hline 1800 & -4.16599 & -0.99237 & -4.16599 & -0.213664757 \\
\hline 2400 & -3.87254 & -1.28582 & -3.87254 & -0.286708077 \\
\hline 3000 & -3.74668 & -1.41168 & -3.74668 & -0.319748585 \\
\hline 3600 & -3.41613 & -1.74223 & -3.41613 & -0.412110368 \\
\hline 4200 & -3.26156 & -1.8968 & -3.26156 & -0.458413091 \\
\hline 4800 & -3.02691 & -2.13145 & -3.02691 & -0.533076402 \\
\hline 5400 & -2.73804 & -2.42032 & -2.73804 & -0.633376364 \\
\hline 6000 & -2.52454 & -2.63382 & -2.52454 & -0.714559832 \\
\hline
\end{tabular}




\begin{tabular}{|c|c|c|c|c|}
\hline 6600 & -2.64611 & -2.51225 & -2.64611 & -0.667528063 \\
\hline 7200 & -2.17714 & -2.98122 & -2.17714 & -0.862606611 \\
\hline 7800 & -2.14414 & -3.01422 & -2.14414 & -0.87788016 \\
\hline 8400 & -1.90041 & -3.25795 & -1.90041 & -0.998549047 \\
\hline 9000 & -1.80705 & -3.35131 & -1.80705 & -1.048923018 \\
\hline 9600 & -1.65843 & -3.49993 & -1.65843 & -1.134747328 \\
\hline 10200 & -1.51458 & -3.64378 & -1.51458 & -1.225480527 \\
\hline 10800 & -1.2844 & -3.87396 & -1.2844 & -1.390327016 \\
\hline 11400 & -1.39035 & -3.76801 & -1.39035 & -1.311063186 \\
\hline 12000 & -1.23769 & -3.92067 & -1.23769 & -1.42737196 \\
\hline 12600 & -1.12606 & -4.0323 & -1.12606 & -1.521893885 \\
\hline 13200 & -1.12139 & -4.03697 & -1.12139 & -1.526049712 \\
\hline 13800 & -0.984191 & -4.174169 & -0.984191 & -1.656553995 \\
\hline 14400 & -0.942356 & -4.216004 & -0.942356 & -1.699990856 \\
\hline 15000 & -0.920187 & -4.238173 & -0.920187 & -1.723797068 \\
\hline 15600 & -0.732226 & -4.426134 & -0.732226 & -1.952284769 \\
\hline 16200 & -0.621112 & -4.537248 & -0.621112 & -2.116862559 \\
\hline 16800 & -0.594066 & -4.564294 & -0.594066 & -2.161383554 \\
\hline 17400 & -0.583406 & -4.574954 & -0.583406 & -2.179490637 \\
\hline
\end{tabular}



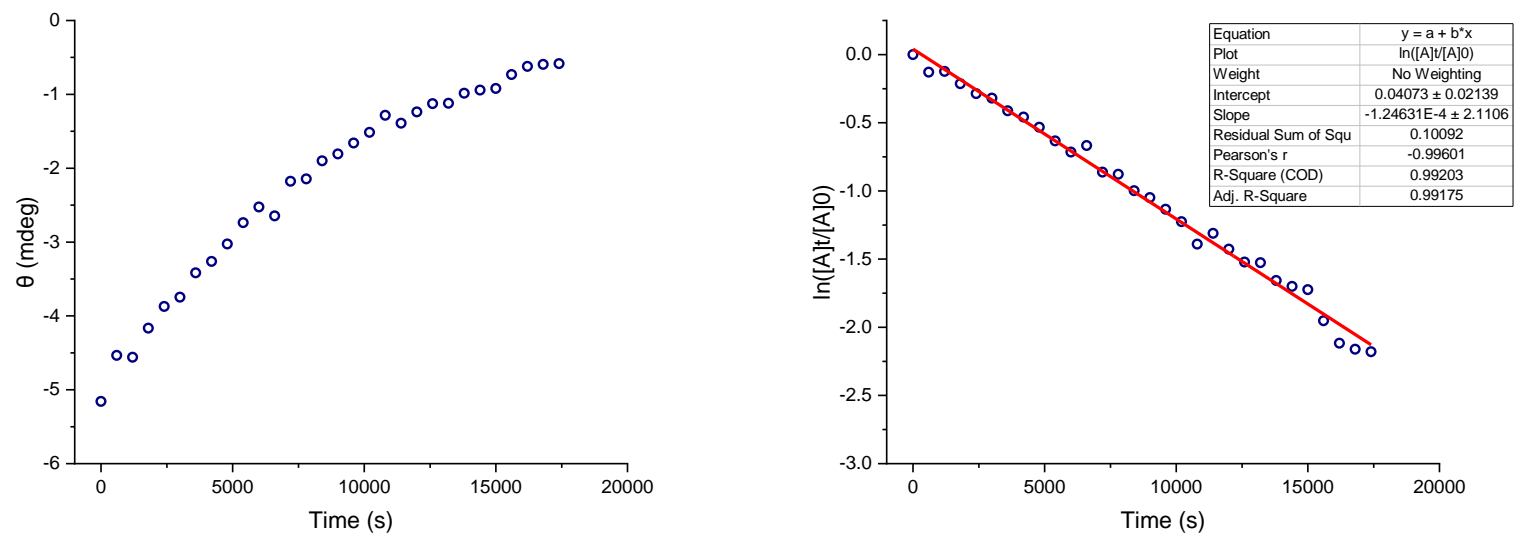

Figure S5: CD measurements of compound $P-2 \mathbf{a}$ at $20^{\circ} \mathrm{C}$ and determination of $k_{r a c}$.

Table S6: Mdeg values of compound $P-2 a$ at $242 \mathrm{~nm}$ at $25^{\circ} \mathrm{C}$.

\begin{tabular}{|l|l|l|l|l|}
\hline time $[\mathrm{s}]$ & Intensity A (mdeg) & Intensity B (mdeg) & {$[A]_{t}$} & \multicolumn{1}{l|}{$\ln \left(\frac{[A]_{t}}{[A]_{0}}\right)$} \\
\hline 0 & -7.95221 & 0 & -7.95221 & 0 \\
\hline 600 & -6.8972 & -1.05501 & -6.8972 & -0.142334345 \\
\hline 1200 & -6.02894 & -1.92327 & -6.02894 & -0.27687867 \\
\hline 1800 & -5.61832 & -2.33389 & -5.61832 & -0.347417191 \\
\hline 2400 & -5.02068 & -2.93153 & -5.02068 & -0.459884495 \\
\hline 3000 & -4.50334 & -3.44887 & -4.50334 & -0.568630534 \\
\hline 3600 & -4.20286 & -3.74935 & -4.20286 & -0.637684632 \\
\hline 4200 & -3.64478 & -4.30743 & -3.64478 & -0.78015387 \\
\hline 4800 & -3.36225 & -4.58996 & -3.36225 & -0.860839485 \\
\hline 5400 & -2.95819 & -4.99402 & -2.95819 & -0.988872283 \\
\hline 6000 & -2.49854 & -5.45367 & -2.49854 & -1.157743316 \\
\hline 6600 & -2.45543 & -5.49678 & -2.45543 & -1.175147979 \\
\hline 7200 & -2.18221 & -5.77 & -2.18221 & -1.293111753 \\
\hline 7800 & -2.0815 & -5.87071 & -2.0815 & -1.34036109 \\
\hline
\end{tabular}




\begin{tabular}{|l|l|l|l|l|}
\hline 8400 & -1.80734 & -6.14487 & -1.80734 & -1.481593726 \\
\hline 9000 & -1.8527 & -6.09951 & -1.8527 & -1.456805843 \\
\hline 9600 & -1.44763 & -6.50458 & -1.44763 & -1.703522141 \\
\hline 10200 & -1.52777 & -6.42444 & -1.52777 & -1.649640722 \\
\hline 10800 & -1.15176 & -6.80045 & -1.15176 & -1.93215867 \\
\hline 11400 & -1.14754 & -6.80467 & -1.14754 & -1.935829357 \\
\hline 12000 & -1.04816 & -6.90405 & -1.04816 & -2.026413631 \\
\hline 12600 & -0.923669 & -7.028541 & -0.923669 & -2.152851374 \\
\hline 13200 & -0.943917 & -7.008293 & -0.943917 & -2.131166918 \\
\hline 13800 & -0.886309 & -7.065901 & -0.886309 & -2.194139508 \\
\hline 14400 & -0.677891 & -7.274319 & -0.677891 & -2.462218648 \\
\hline 15000 & -0.713214 & -7.238996 & -0.713214 & -2.411423641 \\
\hline
\end{tabular}
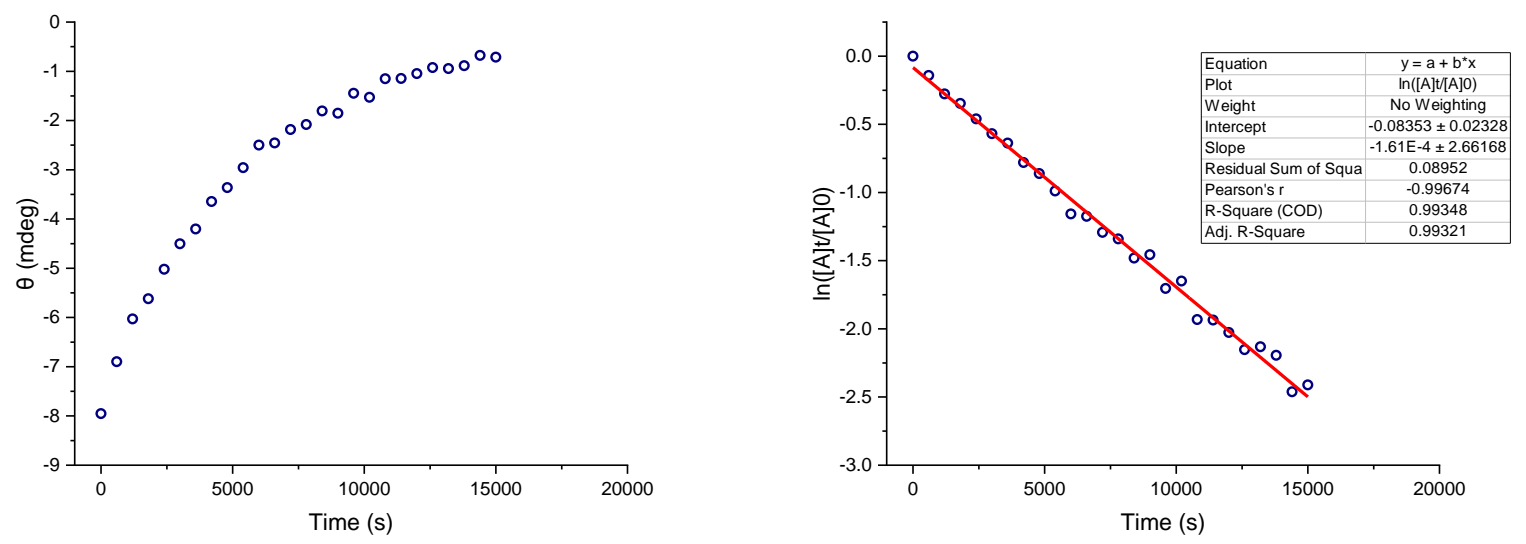

Figure S6: CD measurements of compound $P-2 \mathbf{a}$ at $25^{\circ} \mathrm{C}$ and determination of $k_{r a c}$.

Table S7: Mdeg values of compound $P-2 \mathrm{a}$ at $242 \mathrm{~nm}$ at $30^{\circ} \mathrm{C}$.

\begin{tabular}{|l|l|l|c|c|}
\hline time [s] & Intensity A (mdeg) & Intensity B (mdeg) & {$[A]_{t}$} & $\ln \left(\frac{[A]_{t}}{[A]_{0}}\right)$ \\
\hline 0 & -7.73354 & 0 & -7.73354 & 0 \\
\hline
\end{tabular}




\begin{tabular}{|c|c|c|c|c|}
\hline 600 & -6.50186 & -1.23168 & -6.50186 & -0.173478424 \\
\hline 1200 & -5.46691 & -2.26663 & -5.46691 & -0.346853156 \\
\hline 1800 & -4.79993 & -2.93361 & -4.79993 & -0.476965379 \\
\hline 2400 & -3.94707 & -3.78647 & -3.94707 & -0.672593182 \\
\hline 3000 & -3.23061 & -4.50293 & -3.23061 & -0.87289574 \\
\hline 3600 & -2.86254 & -4.871 & -2.86254 & -0.993857371 \\
\hline 4200 & -2.34405 & -5.38949 & -2.34405 & -1.193686511 \\
\hline 4800 & -1.90385 & -5.82969 & -1.90385 & -1.401688562 \\
\hline 5400 & -1.31528 & -6.41826 & -1.31528 & -1.771517143 \\
\hline 6000 & -1.42749 & -6.30605 & -1.42749 & -1.689649057 \\
\hline 6600 & -1.16782 & -6.56572 & -1.16782 & -1.890427951 \\
\hline 7200 & -1.04105 & -6.69249 & -1.04105 & -2.005336895 \\
\hline 7800 & -0.955086 & -6.778454 & -0.955086 & -2.091520604 \\
\hline 8400 & -0.675741 & -7.057799 & -0.675741 & -2.437512126 \\
\hline 9000 & -0.680291 & -7.053249 & -0.680291 & -2.430801345 \\
\hline 9600 & -0.397845 & -7.335695 & -0.397845 & -2.967259511 \\
\hline 10200 & -0.308849 & -7.424691 & -0.308849 & -3.220469508 \\
\hline 10800 & -0.323057 & -7.410483 & -0.323057 & -3.175493215 \\
\hline 11400 & -0.216433 & -7.517107 & -0.216433 & -3.576040962 \\
\hline
\end{tabular}



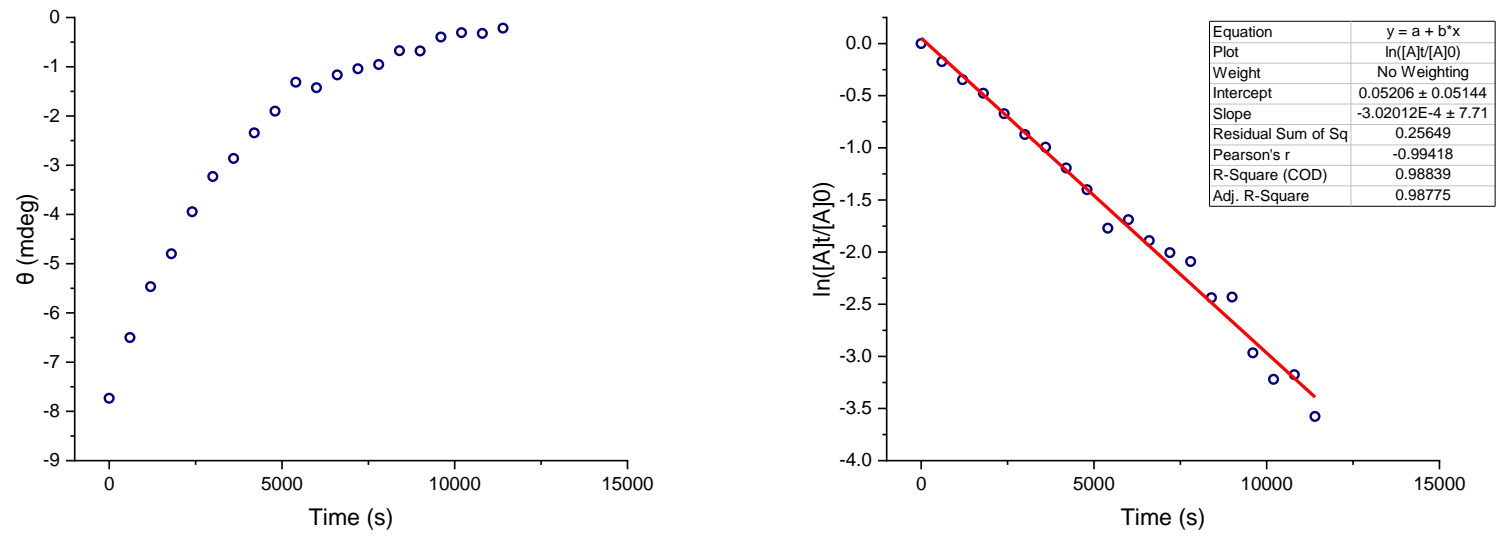

Figure S7: CD measurements of compound $P$-2a at $30{ }^{\circ} \mathrm{C}$ and determination of $k_{\text {rac }}$.

Table S8: Mdeg values of compound $M-1$ a at $248 \mathrm{~nm}$ at $15^{\circ} \mathrm{C}$.

\begin{tabular}{|l|l|l|l|l|}
\hline time [s] & Intensity A (mdeg) & Intensity B (mdeg) & {$[A]_{t}$} & $\ln \left(\frac{[A]_{t}}{[A]_{0}}\right)$ \\
\hline 0 & -6.59045 & 0 & -6.59045 & 0 \\
\hline 600 & -6.62446 & 0.03401 & -6.62446 & 0.005147228 \\
\hline 1200 & -6.52403 & -0.06642 & -6.52403 & -0.010129348 \\
\hline 1800 & -6.57012 & -0.02033 & -6.57012 & -0.003089534 \\
\hline 2400 & -6.47147 & -0.11898 & -6.47147 & -0.018218346 \\
\hline 3000 & -6.48973 & -0.10072 & -6.48973 & -0.015400704 \\
\hline 3600 & -6.55918 & -0.03127 & -6.55918 & -0.004756036 \\
\hline 4200 & -6.33712 & -0.25333 & -6.33712 & -0.039197225 \\
\hline 4800 & -6.40794 & -0.18251 & -6.40794 & -0.028083785 \\
\hline 5400 & -6.50167 & -0.08878 & -6.50167 & -0.013562564 \\
\hline 6000 & -6.46655 & -0.1239 & -6.46655 & -0.018978895 \\
\hline 6600 & -6.31126 & -0.27919 & -6.31126 & -0.043286292 \\
\hline 7200 & -6.27426 & -0.31619 & -6.27426 & -0.049166082 \\
\hline 7800 & -6.25794 & -0.33251 & -6.25794 & -0.051770574 \\
\hline
\end{tabular}




\begin{tabular}{|l|l|l|l|l|}
\hline 8400 & -6.2609 & -0.32955 & -6.2609 & -0.051297687 \\
\hline 9000 & -6.25055 & -0.3399 & -6.25055 & -0.052952172 \\
\hline 9600 & -6.0422 & -0.54825 & -6.0422 & -0.086853447 \\
\hline 10200 & -6.15138 & -0.43907 & -6.15138 & -0.068945185 \\
\hline 10800 & -6.0096 & -0.58085 & -6.0096 & -0.092263441 \\
\hline 11400 & -6.08798 & -0.50247 & -6.08798 & -0.079305296 \\
\hline 12000 & -6.27783 & -0.31262 & -6.27783 & -0.048597252 \\
\hline 12600 & -5.97004 & -0.62041 & -5.97004 & -0.098868004 \\
\hline 13200 & -6.15896 & -0.43149 & -6.15896 & -0.067713699 \\
\hline 13800 & -6.14956 & -0.44089 & -6.14956 & -0.069241097 \\
\hline 14400 & -6.01637 & -0.57408 & -6.01637 & -0.091137544 \\
\hline 15000 & -5.9442 & -0.64625 & -5.9442 & -0.103205677 \\
\hline 15600 & -5.86628 & -0.72417 & -5.86628 & -0.116400929 \\
\hline 16200 & -5.96751 & -0.62294 & -5.96751 & -0.099291876 \\
\hline 16800 & -5.98467 & -0.60578 & -5.98467 & -0.096420432 \\
\hline 17400 & -5.9412 & -0.64925 & -5.9412 & -0.103710498 \\
\hline 18000 & -5.79805 & -0.7924 & -5.79805 & -0.128099977 \\
\hline 18600 & -5.8564 & -0.73405 & -5.8564 & -0.118086551 \\
\hline 19200 & -5.96185 & -0.6286 & -5.96185 & -0.100240796 \\
\hline 19800 & -5.80943 & -0.78102 & -5.80943 & -0.126139172 \\
\hline 20400 & -5.77504 & -0.81541 & -5.77504 & -0.132076449 \\
\hline 21000 & -5.66768 & -0.92277 & -5.66768 & -0.150841769 \\
\hline 21600 & -5.74664 & -0.84381 & -5.74664 & -0.137006295 \\
\hline 22200 & -5.77631 & -0.81414 & -5.77631 & -0.131856561 \\
\hline 22800 & -5.62527 & -0.96518 & -5.62527 & -0.158352685 \\
\hline 23400 & -5.5768 & -1.01365 & -5.5768 & -0.167006496 \\
\hline
\end{tabular}




\begin{tabular}{|l|l|l|l|l|}
\hline 24000 & -5.68551 & -0.90494 & -5.68551 & -0.147700799 \\
\hline 24600 & -5.5185 & -1.07195 & -5.5185 & -0.177515547 \\
\hline 25200 & -5.57776 & -1.01269 & -5.57776 & -0.166834369 \\
\hline 25800 & -5.59032 & -1.00013 & -5.59032 & -0.164585101 \\
\hline 26400 & -5.45584 & -1.13461 & -5.45584 & -0.188935037 \\
\hline 27000 & -5.55859 & -1.03186 & -5.55859 & -0.170277152 \\
\hline 27600 & -5.35116 & -1.23929 & -5.35116 & -0.208308272 \\
\hline 28200 & -5.61832 & -0.97213 & -5.61832 & -0.159588945 \\
\hline 28800 & -5.39466 & -1.19579 & -5.39466 & -0.200212056 \\
\hline 29400 & -5.37453 & -1.21592 & -5.37453 & -0.203950503 \\
\hline 30000 & -5.43631 & -1.15414 & -5.43631 & -0.19252111 \\
\hline 30600 & -5.2719 & -1.31855 & -5.2719 & -0.223230803 \\
\hline 31200 & -5.48164 & -1.10881 & -5.48164 & -0.184217305 \\
\hline 31800 & -5.28278 & -1.30767 & -5.28278 & -0.221169157 \\
\hline 32400 & -5.29367 & -1.29678 & -5.29367 & -0.219109864 \\
\hline 33000 & -5.38725 & -1.2032 & -5.38725 & -0.201586581 \\
\hline 33600 & -5.02905 & -1.5614 & -5.02905 & -0.270390532 \\
\hline 34200 & -5.19384 & -1.39661 & -5.19384 & -0.238148323 \\
\hline 34800 & -5.2128 & -1.37765 & -5.2128 & -0.234504492 \\
\hline 35400 & -5.16741 & -1.42304 & -5.16741 & -0.243250036 \\
\hline 36000 & -5.07613 & -1.51432 & -5.07613 & -0.261072471 \\
\hline 36600 & -5.27369 & -1.31676 & -5.27369 & -0.222891324 \\
\hline 37200 & -5.19732 & -1.39313 & -5.19732 & -0.237478523 \\
\hline 37800 & -4.99461 & -1.59584 & -4.99461 & -0.2772623 \\
\hline 38400 & -5.01423 & -1.57622 & -5.01423 & -0.273341761 \\
\hline 39000 & -5.18327 & -1.40718 & -5.18327 & -0.2401855 \\
\hline
\end{tabular}




\begin{tabular}{|l|l|l|l|l|}
\hline 39600 & -5.19678 & -1.39367 & -5.19678 & -0.237582428 \\
\hline 40200 & -5.11086 & -1.47959 & -5.11086 & -0.254253944 \\
\hline 40800 & -5.19793 & -1.39252 & -5.19793 & -0.237361162 \\
\hline 41400 & -5.06685 & -1.5236 & -5.06685 & -0.262902309 \\
\hline 42000 & -4.93936 & -1.65109 & -4.93936 & -0.288385863 \\
\hline 42600 & -4.79501 & -1.79544 & -4.79501 & -0.318045838 \\
\hline 43200 & -4.88469 & -1.70576 & -4.88469 & -0.299515808 \\
\hline 43800 & -4.94937 & -1.64108 & -4.94937 & -0.286361336 \\
\hline 44400 & -4.87463 & -1.71582 & -4.87463 & -0.301577427 \\
\hline 45000 & -4.81052 & -1.77993 & -4.81052 & -0.314816445 \\
\hline 45600 & -4.99502 & -1.59543 & -4.99502 & -0.277180215 \\
\hline 46200 & -4.78741 & -1.80304 & -4.78741 & -0.319632076 \\
\hline 46800 & -4.76666 & -1.82379 & -4.76666 & -0.323975781 \\
\hline 47400 & -4.71917 & -1.87128 & -4.71917 & -0.333988695 \\
\hline 48000 & -4.74841 & -1.84204 & -4.74841 & -0.327811806 \\
\hline 48600 & -4.75913 & -1.83132 & -4.75913 & -0.325556753 \\
\hline 49200 & -4.80497 & -1.78548 & -4.80497 & -0.315970833 \\
\hline 49800 & -4.63881 & -1.95164 & -4.63881 & -0.351163764 \\
\hline 50400 & -4.56177 & -2.02868 & -4.56177 & -0.367910925 \\
\hline 51000 & -4.66017 & -1.93028 & -4.66017 & -0.346569703 \\
\hline 51600 & -4.7335 & -1.85695 & -4.7335 & -0.330956745 \\
\hline 52200 & -4.75647 & -1.83398 & -4.75647 & -0.326115835 \\
\hline 52800 & -4.60644 & -1.98401 & -4.60644 & -0.358166307 \\
\hline 53400 & -4.64166 & -1.94879 & -4.64166 & -0.350549571 \\
\hline 54000 & -4.72515 & -1.8653 & -4.72515 & -0.332722325 \\
\hline 54600 & -4.59472 & -1.99573 & -4.59472 & -0.360713813 \\
\hline
\end{tabular}



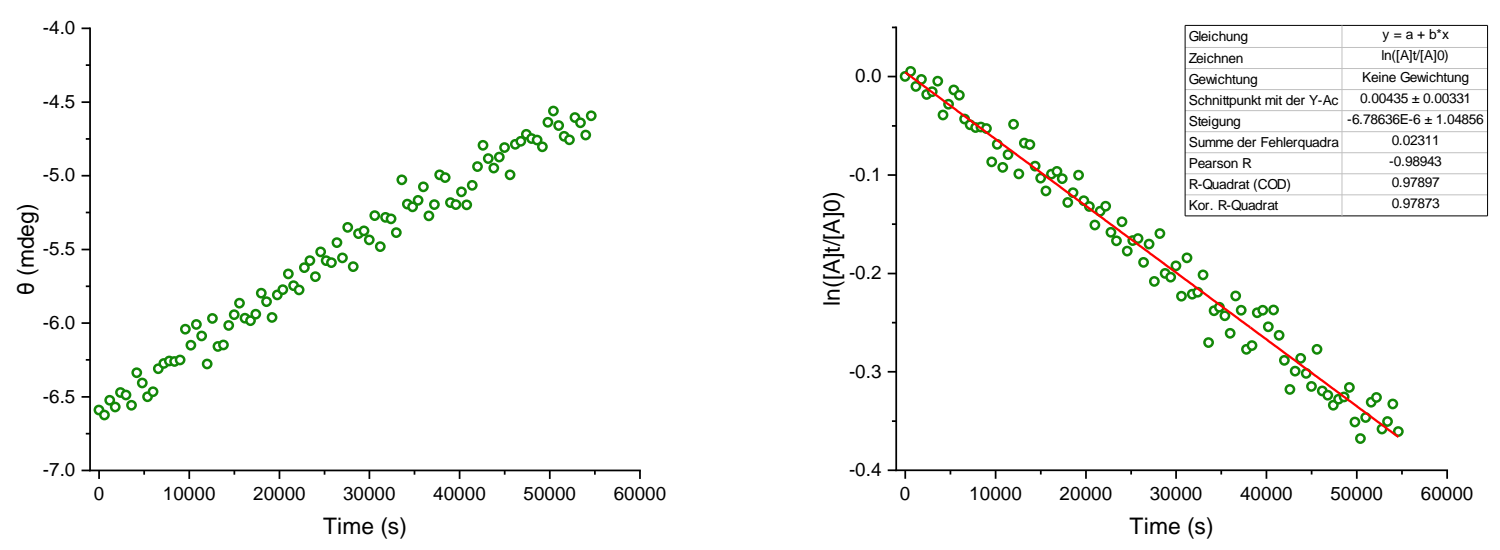

Figure S8: CD measurements of compound $\mathrm{M}-\mathbf{1 a}$ at $15^{\circ} \mathrm{C}$ and determination of $k_{\text {rac }}$.

Table S9: Mdeg values of compound $M-1 a$ at $248 \mathrm{~nm}$ at $20^{\circ} \mathrm{C}$.

\begin{tabular}{|l|l|l|r|c|}
\hline time [s] & Intensity A (mdeg) & Intensity B (mdeg) & {$[A]_{t}$} & $\ln \left(\frac{[A]_{t}}{[A]_{0}}\right)$ \\
\hline 0 & -6.10332 & 0 & -6.10332 & 0 \\
\hline 600 & -6.04306 & -0.06026 & -6.04306 & -0.009922379 \\
\hline 1200 & -6.19088 & 0.08756 & -6.19088 & 0.014244356 \\
\hline 1800 & -6.12848 & 0.02516 & -6.12848 & 0.004113873 \\
\hline 2400 & -6.06422 & -0.0391 & -6.06422 & -0.006426958 \\
\hline 3000 & -5.91946 & -0.18386 & -5.91946 & -0.030587657 \\
\hline 3600 & -5.98019 & -0.12313 & -5.98019 & -0.020380545 \\
\hline 4200 & -5.80784 & -0.29548 & -5.80784 & -0.049624156 \\
\hline 4800 & -5.59889 & -0.50443 & -5.59889 & -0.086264522 \\
\hline 5400 & -5.66663 & -0.43669 & -5.66663 & -0.074238301 \\
\hline 6000 & -5.70498 & -0.39834 & -5.70498 & -0.067493408 \\
\hline 6600 & -5.61312 & -0.4902 & -5.61312 & -0.083726171 \\
\hline 7200 & -5.51742 & -0.5859 & -5.51742 & -0.100922526 \\
\hline 7800 & -5.49823 & -0.60509 & -5.49823 & -0.104406663 \\
\hline
\end{tabular}




\begin{tabular}{|l|l|l|l|l|}
\hline 8400 & -5.54515 & -0.55817 & -5.54515 & -0.095909214 \\
\hline 9000 & -5.43316 & -0.67016 & -5.43316 & -0.116311969 \\
\hline 9600 & -5.39178 & -0.71154 & -5.39178 & -0.123957314 \\
\hline 10200 & -5.17111 & -0.93221 & -5.17111 & -0.16574552 \\
\hline 10800 & -5.35208 & -0.75124 & -5.35208 & -0.131347615 \\
\hline 11400 & -5.20405 & -0.89927 & -5.20405 & -0.159395717 \\
\hline 12000 & -5.36881 & -0.73451 & -5.36881 & -0.128226603 \\
\hline 12600 & -5.1368 & -0.96652 & -5.1368 & -0.172402568 \\
\hline 13200 & -5.15438 & -0.94894 & -5.15438 & -0.168986047 \\
\hline 13800 & -5.22363 & -0.87969 & -5.22363 & -0.155640323 \\
\hline 14400 & -5.11269 & -0.99063 & -5.11269 & -0.177107201 \\
\hline 15000 & -5.09688 & -1.00644 & -5.09688 & -0.180204298 \\
\hline 15600 & -5.11989 & -0.98343 & -5.11989 & -0.175699931 \\
\hline 16200 & -4.96325 & -1.14007 & -4.96325 & -0.206772117 \\
\hline 16800 & -5.05165 & -1.05167 & -5.05165 & -0.189117963 \\
\hline 17400 & -4.67003 & -1.43329 & -4.67003 & -0.26766739 \\
\hline 18000 & -4.93497 & -1.16835 & -4.93497 & -0.212486292 \\
\hline 18600 & -4.80943 & -1.29389 & -4.80943 & -0.238254311 \\
\hline 19200 & -4.78633 & -1.31699 & -4.78633 & -0.243068947 \\
\hline 19800 & -4.72366 & -1.37966 & -4.72366 & -0.256248963 \\
\hline 20400 & -4.74619 & -1.35713 & -4.74619 & -0.251490694 \\
\hline 21000 & -4.60262 & -1.5007 & -4.60262 & -0.282207179 \\
\hline 21600 & -4.6146 & -1.48872 & -4.6146 & -0.279607695 \\
\hline 22200 & -4.62016 & -1.48316 & -4.62016 & -0.278403549 \\
\hline 22800 & -4.66344 & -1.43988 & -4.66344 & -0.269079512 \\
\hline 23400 & -4.56727 & -1.53605 & -4.56727 & -0.289917233 \\
\hline
\end{tabular}




\begin{tabular}{|l|l|l|l|l|}
\hline 24000 & -4.46136 & -1.64196 & -4.46136 & -0.313379233 \\
\hline 24600 & -4.46729 & -1.63603 & -4.46729 & -0.312050925 \\
\hline 25200 & -4.48552 & -1.6178 & -4.48552 & -0.307978455 \\
\hline 25800 & -4.51391 & -1.58941 & -4.51391 & -0.301669145 \\
\hline 26400 & -4.45995 & -1.64337 & -4.45995 & -0.31369533 \\
\hline 27000 & -4.34242 & -1.7609 & -4.34242 & -0.340401089 \\
\hline 27600 & -4.25999 & -1.84333 & -4.25999 & -0.359566073 \\
\hline 28200 & -4.4229 & -1.68042 & -4.4229 & -0.322037296 \\
\hline 28800 & -4.01434 & -2.08898 & -4.01434 & -0.418959935 \\
\hline 29400 & -4.25602 & -1.8473 & -4.25602 & -0.360498434 \\
\hline 30000 & -4.17862 & -1.9247 & -4.17862 & -0.378851837 \\
\hline 30600 & -4.16304 & -1.94028 & -4.16304 & -0.382587309 \\
\hline 31200 & -4.04377 & -2.05955 & -4.04377 & -0.41165546 \\
\hline 31800 & -4.11565 & -1.98767 & -4.11565 & -0.394036105 \\
\hline 32400 & -4.13466 & -1.96866 & -4.13466 & -0.389427785 \\
\hline 33000 & -3.95585 & -2.14747 & -3.95585 & -0.433637389 \\
\hline 33600 & -4.17845 & -1.92487 & -4.17845 & -0.378892521 \\
\hline 34200 & -3.93391 & -2.16941 & -3.93391 & -0.439199043 \\
\hline 34800 & -3.88453 & -2.21879 & -3.88453 & -0.451830887 \\
\hline 35400 & -4.04948 & -2.05384 & -4.04948 & -0.410244408 \\
\hline 36000 & -3.7344 & -2.36892 & -3.7344 & -0.491245722 \\
\hline 36600 & -3.70024 & -2.40308 & -3.70024 & -0.500435203 \\
\hline 37200 & -3.77644 & -2.32688 & -3.77644 & -0.480051118 \\
\hline 37800 & -3.70518 & -2.39814 & -3.70518 & -0.499101045 \\
\hline 38400 & -3.83411 & -2.26921 & -3.83411 & -0.464895551 \\
\hline 39000 & -3.71268 & -2.39064 & -3.71268 & -0.497078898 \\
\hline
\end{tabular}




\begin{tabular}{|l|l|l|l|l|}
\hline 39600 & -3.57008 & -2.53324 & -3.57008 & -0.536244881 \\
\hline 40200 & -3.67289 & -2.43043 & -3.67289 & -0.507854067 \\
\hline 40800 & -3.59981 & -2.50351 & -3.59981 & -0.527951819 \\
\hline 41400 & -3.56636 & -2.53696 & -3.56636 & -0.537287418 \\
\hline 42000 & -3.59204 & -2.51128 & -3.59204 & -0.530112599 \\
\hline 42600 & -3.52211 & -2.58121 & -3.52211 & -0.549772644 \\
\hline 43200 & -3.53356 & -2.56976 & -3.53356 & -0.546527024 \\
\hline 43800 & -3.54332 & -2.56 & -3.54332 & -0.543768745 \\
\hline 44400 & -3.52083 & -2.58249 & -3.52083 & -0.550136128 \\
\hline 45000 & -3.66904 & -2.43428 & -3.66904 & -0.508902838 \\
\hline 45600 & -3.39954 & -2.70378 & -3.39954 & -0.585192757 \\
\hline 46200 & -3.27081 & -2.83251 & -3.27081 & -0.623795225 \\
\hline 46800 & -3.14996 & -2.95336 & -3.14996 & -0.661443131 \\
\hline 47400 & -3.33088 & -2.77244 & -3.33088 & -0.605596352 \\
\hline 48000 & -3.4088 & -2.69452 & -3.4088 & -0.582472562 \\
\hline 48600 & -3.46804 & -2.63528 & -3.46804 & -0.565243293 \\
\hline 49200 & -3.17011 & -2.93321 & -3.17011 & -0.655066598 \\
\hline 49800 & -3.22604 & -2.87728 & -3.22604 & -0.637577507 \\
\hline 50400 & -3.0822 & -3.02112 & -3.0822 & -0.683189258 \\
\hline 51000 & -3.19623 & -2.90709 & -3.19623 & -0.646860895 \\
\hline 51600 & -3.19094 & -2.91238 & -3.19094 & -0.648517341 \\
\hline 52200 & -2.9824 & -3.12092 & -2.9824 & -0.71610454 \\
\hline 52800 & -2.95874 & -3.14458 & -2.95874 & -0.724069383 \\
\hline 53400 & -3.16632 & -2.937 & -3.16632 & -0.656262855 \\
\hline 54000 & -3.17288 & -2.93044 & -3.17288 & -0.654193193 \\
\hline 54600 & -2.94039 & -3.16293 & -2.94039 & -0.73029066 \\
\hline & & & & \\
\hline & & & & \\
\hline & & & & \\
\hline
\end{tabular}




\begin{tabular}{|l|l|l|l|l|}
\hline 55200 & -2.99705 & -3.10627 & -2.99705 & -0.711204414 \\
\hline 55800 & -3.02349 & -3.07983 & -3.02349 & -0.702421092 \\
\hline 56400 & -2.97617 & -3.12715 & -2.97617 & -0.718195646 \\
\hline 57000 & -2.95092 & -3.1524 & -2.95092 & -0.726715899 \\
\hline 57600 & -2.99513 & -3.10819 & -2.99513 & -0.711845249 \\
\hline
\end{tabular}
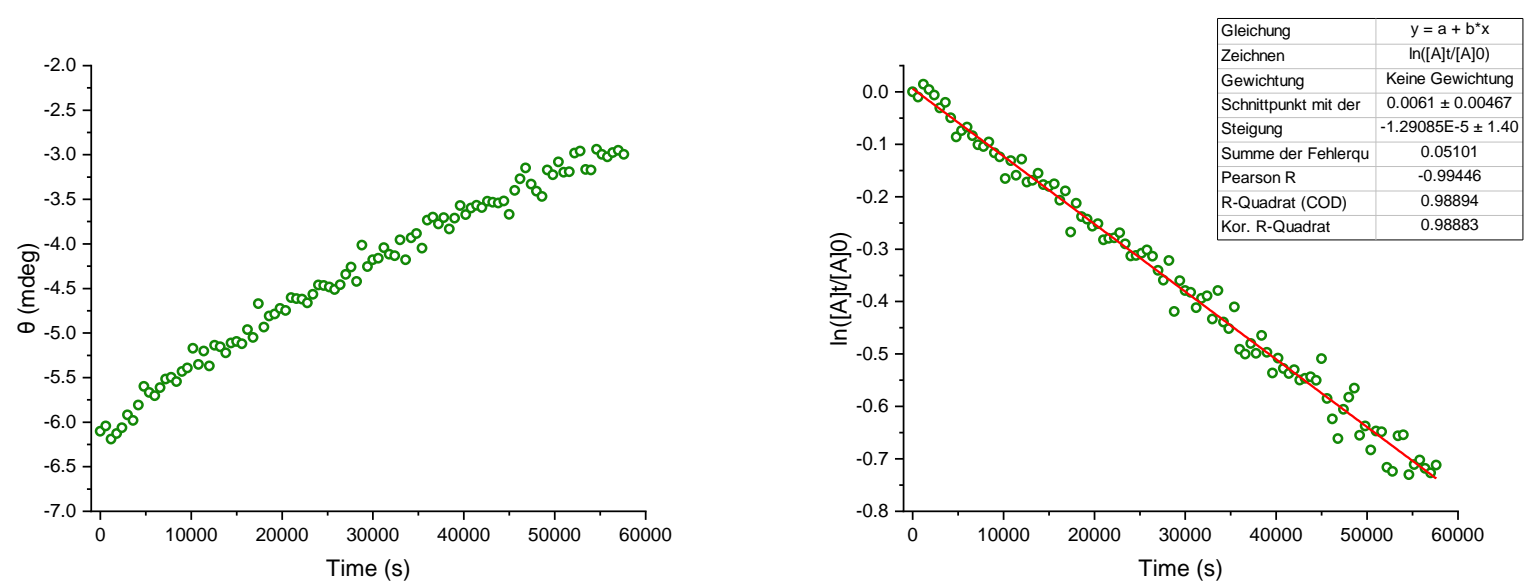

Figure S9: CD measurements of compound $M-1 \mathrm{a}$ at $20^{\circ} \mathrm{C}$ and determination of $k_{r a c}$.

Table S10: Mdeg values of compound $M-1 \mathrm{a}$ at $248 \mathrm{~nm}$ at $25^{\circ} \mathrm{C}$.

\begin{tabular}{|l|l|l|c|c|}
\hline time [s] & Intensity A (mdeg) & Intensity B (mdeg) & {$[A]_{t}$} & $\ln \left(\frac{[A]_{t}}{[A]_{0}}\right)$ \\
\hline 0 & -5.1656 & 0 & -5.1656 & 0 \\
\hline 600 & -5.03738 & -0.12822 & -5.03738 & -0.025135157 \\
\hline 1200 & -4.98262 & -0.18298 & -4.98262 & -0.036065405 \\
\hline 1800 & -4.75071 & -0.41489 & -4.75071 & -0.083727182 \\
\hline 2400 & -4.71484 & -0.45076 & -4.71484 & -0.091306281 \\
\hline 3000 & -4.65244 & -0.51316 & -4.65244 & -0.104629449 \\
\hline 3600 & -4.49698 & -0.66862 & -4.49698 & -0.138615202 \\
\hline 4200 & -4.55346 & -0.61214 & -4.55346 & -0.126133879 \\
\hline
\end{tabular}




\begin{tabular}{|l|l|l|l|l|}
\hline 4800 & -4.43922 & -0.72638 & -4.43922 & -0.151542577 \\
\hline 5400 & -4.39945 & -0.76615 & -4.39945 & -0.160541729 \\
\hline 6000 & -4.35243 & -0.81317 & -4.35243 & -0.171286953 \\
\hline 6600 & -4.30462 & -0.86098 & -4.30462 & -0.182332398 \\
\hline 7200 & -4.24779 & -0.91781 & -4.24779 & -0.195622415 \\
\hline 7800 & -4.17587 & -0.98973 & -4.17587 & -0.212698543 \\
\hline 8400 & -4.14567 & -1.01993 & -4.14567 & -0.219956846 \\
\hline 9000 & -4.03703 & -1.12857 & -4.03703 & -0.246511989 \\
\hline 9600 & -4.08417 & -1.08143 & -4.08417 & -0.234902737 \\
\hline 10200 & -3.91278 & -1.25282 & -3.91278 & -0.277773144 \\
\hline 10800 & -3.89907 & -1.26653 & -3.89907 & -0.281283199 \\
\hline 11400 & -3.88503 & -1.28057 & -3.88503 & -0.284890557 \\
\hline 12000 & -3.64295 & -1.52265 & -3.64295 & -0.349227469 \\
\hline 12600 & -3.64398 & -1.52162 & -3.64398 & -0.348944771 \\
\hline 13200 & -3.50218 & -1.66342 & -3.50218 & -0.388635631 \\
\hline 13800 & -3.6846 & -1.481 & -3.6846 & -0.337859291 \\
\hline 14400 & -3.58848 & -1.57712 & -3.58848 & -0.364292548 \\
\hline 15000 & -3.49661 & -1.66899 & -3.49661 & -0.390227335 \\
\hline 15600 & -3.40304 & -1.76256 & -3.40304 & -0.417352113 \\
\hline 16200 & -3.50943 & -1.65617 & -3.50943 & -0.386567631 \\
\hline 16800 & -3.37802 & -1.78758 & -3.37802 & -0.424731523 \\
\hline 17400 & -3.18803 & -1.97757 & -3.18803 & -0.482618091 \\
\hline 18000 & -3.28572 & -1.87988 & -3.28572 & -0.452435456 \\
\hline 18600 & -3.24866 & -1.91694 & -3.24866 & -0.463778659 \\
\hline 19200 & -3.27515 & -1.89045 & -3.27515 & -0.455657593 \\
\hline 19800 & -3.11643 & -2.04917 & -3.11643 & -0.505333146 \\
\hline
\end{tabular}




\begin{tabular}{|l|l|l|l|l|}
\hline 20400 & -2.98874 & -2.17686 & -2.98874 & -0.547169368 \\
\hline 21000 & -2.89448 & -2.27112 & -2.89448 & -0.579215787 \\
\hline 21600 & -2.87842 & -2.28718 & -2.87842 & -0.58477973 \\
\hline 22200 & -2.82418 & -2.34142 & -2.82418 & -0.603803205 \\
\hline 22800 & -2.84825 & -2.31735 & -2.84825 & -0.595316492 \\
\hline 23400 & -2.73642 & -2.42918 & -2.73642 & -0.635370766 \\
\hline 24000 & -2.87032 & -2.29528 & -2.87032 & -0.587597741 \\
\hline 24600 & -2.56072 & -2.60488 & -2.56072 & -0.701732793 \\
\hline 25200 & -2.61101 & -2.55459 & -2.61101 & -0.682284143 \\
\hline 25800 & -2.69602 & -2.46958 & -2.69602 & -0.650244651 \\
\hline 26400 & -2.57603 & -2.58957 & -2.57603 & -0.695771808 \\
\hline 27000 & -2.63826 & -2.52734 & -2.63826 & -0.671901653 \\
\hline 27600 & -2.48855 & -2.67705 & -2.48855 & -0.730321051 \\
\hline 28200 & -2.36198 & -2.80362 & -2.36198 & -0.782521012 \\
\hline 28800 & -2.48082 & -2.68478 & -2.48082 & -0.733432112 \\
\hline 29400 & -2.34321 & -2.82239 & -2.34321 & -0.790499478 \\
\hline 30000 & -2.23222 & -2.93338 & -2.23222 & -0.839024656 \\
\hline 30600 & -2.31399 & -2.85161 & -2.31399 & -0.803047955 \\
\hline 31200 & -2.22427 & -2.94133 & -2.22427 & -0.84259249 \\
\hline 31800 & -2.12144 & -3.04416 & -2.12144 & -0.889926159 \\
\hline 32400 & -2.11997 & -3.04563 & -2.11997 & -0.890619325 \\
\hline 33000 & -2.0548 & -3.1108 & -2.0548 & -0.921842743 \\
\hline 33600 & -2.13255 & -3.03305 & -2.13255 & -0.884702816 \\
\hline 34200 & -2.04604 & -3.11956 & -2.04604 & -0.926115045 \\
\hline 34800 & -1.97836 & -3.18724 & -1.97836 & -0.959753044 \\
\hline 35400 & -1.9757 & -3.1899 & -1.9757 & -0.961098496 \\
\hline
\end{tabular}




\begin{tabular}{|l|l|l|l|l|}
\hline 36000 & -2.06096 & -3.10464 & -2.06096 & -0.918849369 \\
\hline 36600 & -1.9765 & -3.1891 & -1.9765 & -0.960693659 \\
\hline 37200 & -2.09337 & -3.07223 & -2.09337 & -0.903246055 \\
\hline 37800 & -1.96352 & -3.20208 & -1.96352 & -0.967282481 \\
\hline 38400 & -1.92046 & -3.24514 & -1.92046 & -0.989456522 \\
\hline 39000 & -1.83922 & -3.32638 & -1.83922 & -1.032679694 \\
\hline 39600 & -1.73409 & -3.43151 & -1.73409 & -1.091538482 \\
\hline 40200 & -1.80261 & -3.36299 & -1.80261 & -1.052785648 \\
\hline 40800 & -1.76583 & -3.39977 & -1.76583 & -1.073400428 \\
\hline 41400 & -1.72382 & -3.44178 & -1.72382 & -1.097478504 \\
\hline 42000 & -1.59641 & -3.56919 & -1.59641 & -1.174263904 \\
\hline 42600 & -1.67383 & -3.49177 & -1.67383 & -1.126906849 \\
\hline 43200 & -1.76333 & -3.40227 & -1.76333 & -1.074817195 \\
\hline 43800 & -1.4851 & -3.6805 & -1.4851 & -1.246539152 \\
\hline 44400 & -1.55627 & -3.60933 & -1.55627 & -1.19972933 \\
\hline 45000 & -1.51487 & -3.65073 & -1.51487 & -1.226691636 \\
\hline 45600 & -1.54517 & -3.62043 & -1.54517 & -1.206887326 \\
\hline 46200 & -1.46168 & -3.70392 & -1.46168 & -1.262434803 \\
\hline 46800 & -1.4125 & -3.7531 & -1.4125 & -1.296660078 \\
\hline 47400 & -1.38511 & -3.78049 & -1.38511 & -1.316241703 \\
\hline 48000 & -1.38516 & -3.78044 & -1.38516 & -1.316205606 \\
\hline 48600 & -1.38505 & -3.78055 & -1.38505 & -1.316285022 \\
\hline & & & & \\
\hline & & & & \\
\hline
\end{tabular}



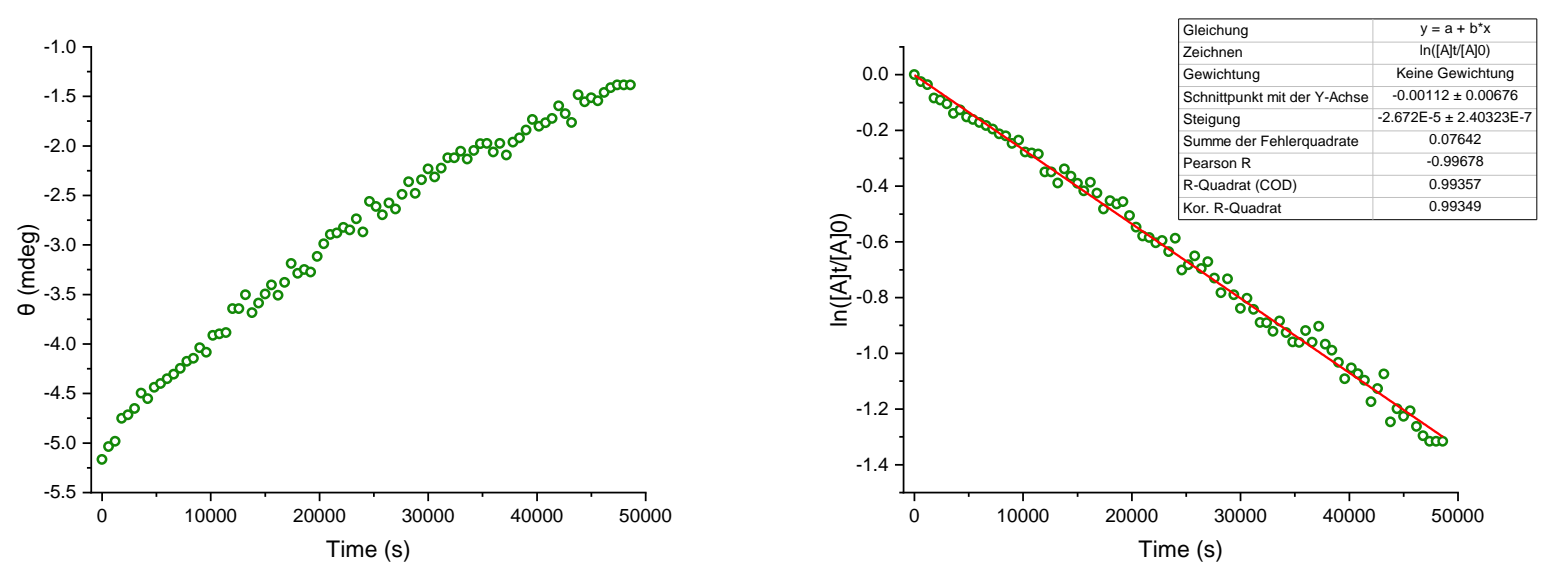

Figure S10: CD measurements of compound $M-1 \mathbf{a}$ at $25^{\circ} \mathrm{C}$ and determination of $k_{\text {rac }}$.

Table S11: Mdeg values of compound $M-1$ a at $248 \mathrm{~nm}$ at $30^{\circ} \mathrm{C}$.

\begin{tabular}{|l|l|l|c|c|}
\hline time [s] & Intensity A (mdeg) & Intensity B (mdeg) & {$[A]_{t}$} & $\ln \left(\frac{[A]_{t}}{[A]_{0}}\right)$ \\
\hline 0 & -4.91992 & 0 & -4.91992 & 0 \\
\hline 600 & -4.64124 & -0.27868 & -4.64124 & -0.058310698 \\
\hline 1200 & -4.38412 & -0.5358 & -4.38412 & -0.115303349 \\
\hline 1800 & -4.22966 & -0.69026 & -4.22966 & -0.151170659 \\
\hline 2400 & -4.35694 & -0.56298 & -4.35694 & -0.121522294 \\
\hline 3000 & -4.11742 & -0.8025 & -4.11742 & -0.178065517 \\
\hline 3600 & -4.05323 & -0.86669 & -4.05323 & -0.193778176 \\
\hline 4200 & -4.06936 & -0.85056 & -4.06936 & -0.189806531 \\
\hline 4800 & -3.89648 & -1.02344 & -3.89648 & -0.233218689 \\
\hline 5400 & -3.8295 & -1.09042 & -3.8295 & -0.250558024 \\
\hline 6000 & -3.82166 & -1.09826 & -3.82166 & -0.252607387 \\
\hline 6600 & -3.63515 & -1.28477 & -3.63515 & -0.302641895 \\
\hline 7200 & -3.58505 & -1.33487 & -3.58505 & -0.316519849 \\
\hline 7800 & -3.41659 & -1.50333 & -3.41659 & -0.364649293 \\
\hline
\end{tabular}




\begin{tabular}{|l|l|l|l|l|}
\hline 8400 & -3.28596 & -1.63396 & -3.28596 & -0.403633424 \\
\hline 9000 & -3.22379 & -1.69613 & -3.22379 & -0.422734584 \\
\hline 9600 & -3.34266 & -1.57726 & -3.34266 & -0.386525373 \\
\hline 10200 & -3.14654 & -1.77338 & -3.14654 & -0.446988834 \\
\hline 10800 & -2.9262 & -1.99372 & -2.9262 & -0.519587617 \\
\hline 11400 & -2.99854 & -1.92138 & -2.99854 & -0.495166767 \\
\hline 12000 & -3.06464 & -1.85528 & -3.06464 & -0.473362163 \\
\hline 12600 & -2.96909 & -1.95083 & -2.96909 & -0.505036762 \\
\hline 13200 & -2.70254 & -2.21738 & -2.70254 & -0.599100199 \\
\hline 13800 & -2.71535 & -2.20457 & -2.71535 & -0.594371412 \\
\hline 14400 & -2.7776 & -2.14232 & -2.7776 & -0.571705025 \\
\hline 15000 & -2.49256 & -2.42736 & -2.49256 & -0.679981975 \\
\hline 15600 & -2.4256 & -2.49432 & -2.4256 & -0.707213354 \\
\hline 16200 & -2.50264 & -2.41728 & -2.50264 & -0.675946096 \\
\hline 16800 & -2.26743 & -2.65249 & -2.26743 & -0.774645239 \\
\hline 17400 & -2.26708 & -2.65284 & -2.26708 & -0.77479961 \\
\hline 18000 & -2.18598 & -2.73394 & -2.18598 & -0.81122803 \\
\hline 18600 & -2.05158 & -2.86834 & -2.05158 & -0.874682042 \\
\hline 19200 & -2.03842 & -2.8815 & -2.03842 & -0.881117272 \\
\hline 19800 & -2.05437 & -2.86555 & -2.05437 & -0.873323039 \\
\hline 20400 & -2.01347 & -2.90645 & -2.01347 & -0.893432668 \\
\hline 21000 & -1.98595 & -2.93397 & -1.98595 & -0.907194881 \\
\hline 21600 & -1.92422 & -2.9957 & -1.92422 & -0.938771579 \\
\hline 22200 & -1.9548 & -2.96512 & -1.9548 & -0.923004384 \\
\hline 22800 & -1.73726 & -3.18266 & -1.73726 & -1.040983111 \\
\hline 23400 & -1.6483 & -3.27162 & -1.6483 & -1.093547816 \\
\hline
\end{tabular}




\begin{tabular}{|l|l|l|l|l|}
\hline 24000 & -1.69472 & -3.2252 & -1.69472 & -1.065774735 \\
\hline 24600 & -1.65422 & -3.2657 & -1.65422 & -1.089962672 \\
\hline 25200 & -1.64009 & -3.27983 & -1.64009 & -1.098541152 \\
\hline 25800 & -1.52645 & -3.39347 & -1.52645 & -1.170347492 \\
\hline 26400 & -1.50007 & -3.41985 & -1.50007 & -1.187780497 \\
\hline 27000 & -1.57448 & -3.34544 & -1.57448 & -1.139367211 \\
\hline 27600 & -1.62226 & -3.29766 & -1.62226 & -1.109472031 \\
\hline 28200 & -1.3547 & -3.56522 & -1.3547 & -1.289712243 \\
\hline 28800 & -1.41729 & -3.50263 & -1.41729 & -1.244545673 \\
\hline 29400 & -1.31121 & -3.60871 & -1.31121 & -1.322341895 \\
\hline 30000 & -1.38584 & -3.53408 & -1.38584 & -1.266985816 \\
\hline 30600 & -1.21276 & -3.70716 & -1.21276 & -1.400393516 \\
\hline 31200 & -1.18216 & -3.73776 & -1.18216 & -1.425948997 \\
\hline 31800 & -1.23488 & -3.68504 & -1.23488 & -1.382318471 \\
\hline 32400 & -1.24103 & -3.67889 & -1.24103 & -1.37735059 \\
\hline
\end{tabular}
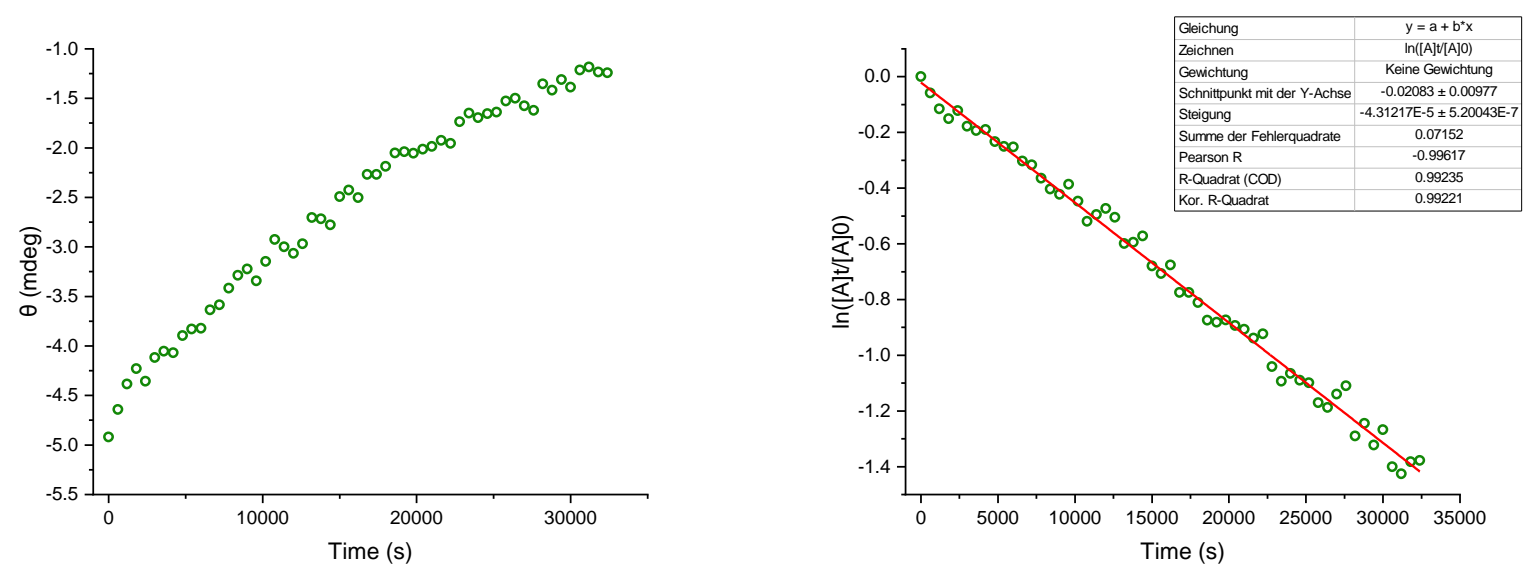

Figure S11: CD measurements of compound $M-1 \mathrm{a}$ at $30^{\circ} \mathrm{C}$ and determination of $k_{\text {rac }}$. 
Table S12: Rate constants and half-lifes of compound $\mathbf{1 a}$ and $\mathbf{2 a}$ at the different temperatures.

\begin{tabular}{|c|c|c|c|c|c|c|c|c|}
\hline \multirow{2}{*}{$\begin{array}{c}\text { Temperature } \\
15^{\circ} \mathrm{C}\end{array}$} & \multicolumn{2}{|c|}{$\begin{array}{l}\text { Rate constant }[1 / \mathrm{s}] \text { of } \\
\text { compound } 1 \mathrm{a}\end{array}$} & \multicolumn{2}{|c|}{ Half-life [h] } & \multicolumn{2}{|c|}{$\begin{array}{l}\text { Rate constant }[1 / \mathrm{s}] \text { of } \\
\text { compound } 2 \mathrm{a}\end{array}$} & \multicolumn{2}{|c|}{ Half-life [h] } \\
\hline & $k_{\text {en }}=$ & $3.39 \times 10^{-6}$ & $t_{e n}=$ & 56.74 & $k_{\text {en }}=$ & $3.29 \times 10^{-5}$ & $t_{e n}=$ & 5.86 \\
\hline $20^{\circ} \mathrm{C}$ & $k_{e n}=$ & $6.45 \times 10^{-6}$ & $t_{e n}=$ & 29.83 & $k_{e n}=$ & $6.23 \times 10^{-5}$ & $t_{e n}=$ & 3.09 \\
\hline $25^{\circ} \mathrm{C}$ & $k_{\text {en }}=$ & $1.34 \times 10^{-5}$ & $t_{e n}=$ & 14.41 & $k_{\text {en }}=$ & $8.05 \times 10^{-5}$ & $t_{e n}=$ & 2.39 \\
\hline $30^{\circ} \mathrm{C}$ & $k_{\text {en }}=$ & $2.16 \times 10^{-5}$ & $t_{e n}=$ & 8.93 & $k_{\text {en }}=$ & $1.51 \times 10^{-4}$ & $t_{e n}=$ & 1.28 \\
\hline
\end{tabular}

Table S13: Calculated values for the free Gibbs energy for compound 1a and 2a.

\begin{tabular}{|c|r|r|}
\hline$\Delta G_{e n(T)}^{\neq}[\mathrm{kJ} / \mathrm{mol}]$ & Compound 1a & Compound 2a \\
\hline$\Delta G_{e n(288.15 K)}^{\neq}$ & 100.7 & 95.2 \\
\hline$\Delta G_{e n(288.15 K)}^{\neq}$ & 100.9 & 95.4 \\
\hline$\Delta G_{e n(288.15 K)}^{\neq}$ & 100.8 & 96.4 \\
\hline$\Delta G_{e n(288.15 K)}^{\neq}$ & 101.4 & 96.5 \\
\hline
\end{tabular}

Table S14: The obtained $\Delta H_{e n}^{\neq}$and $\Delta S_{e n}^{\neq}$values for compound 1a and $\mathbf{2 a}$.

\begin{tabular}{|l|r|r|}
\cline { 2 - 3 } \multicolumn{1}{c|}{} & Compound 1a & Compound 2a \\
\hline$\Delta H_{e n}^{\neq}[\mathrm{kJ} / \mathrm{mol}]$ & 88.8 & 67.7 \\
\hline$\Delta S_{e n}^{\neq}\left[\mathrm{J} / \mathrm{mol}^{*} \mathrm{~K}\right]$ & -35.5 & -89.5 \\
\hline
\end{tabular}




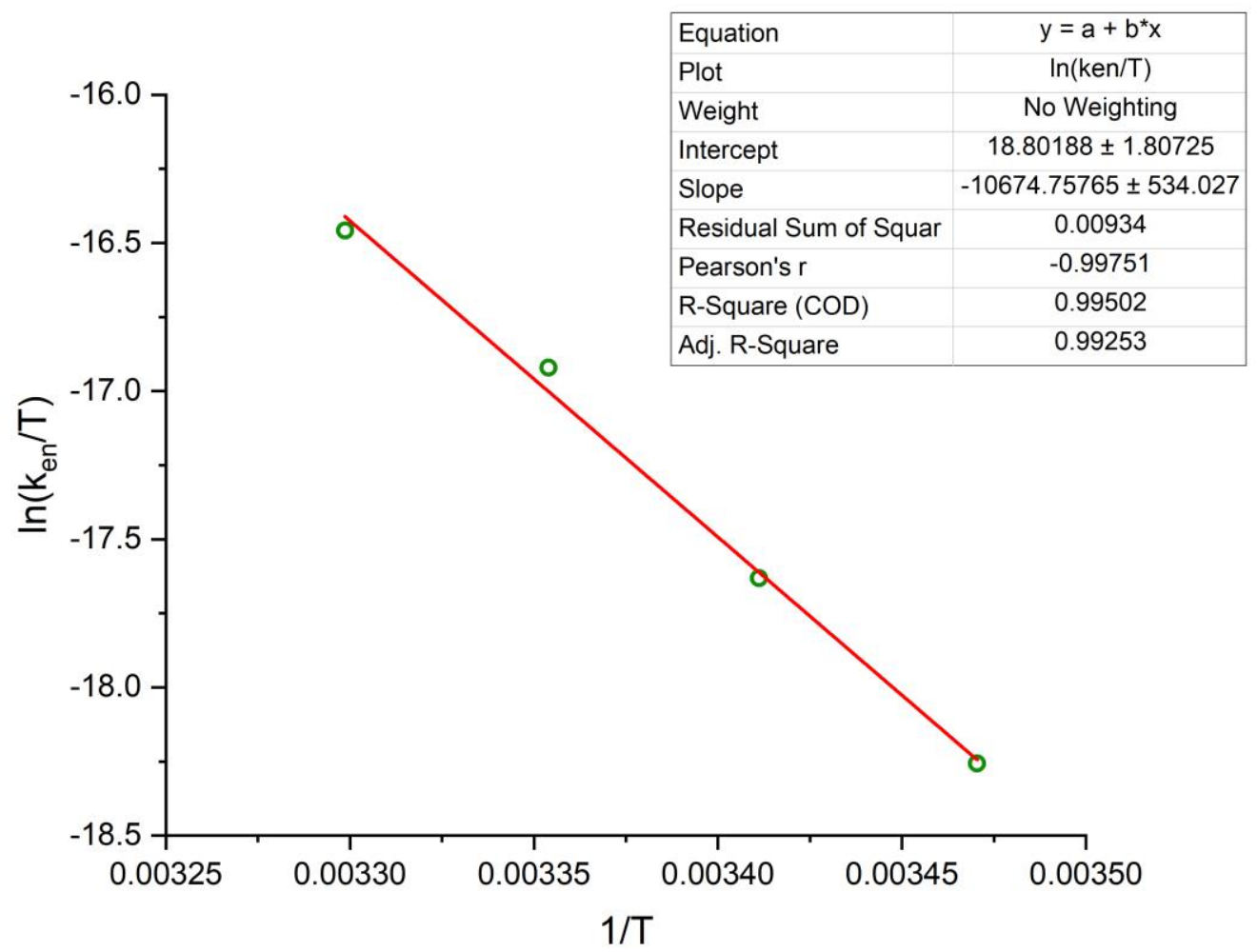

Figure S12: Eyring plot of compound 1a.

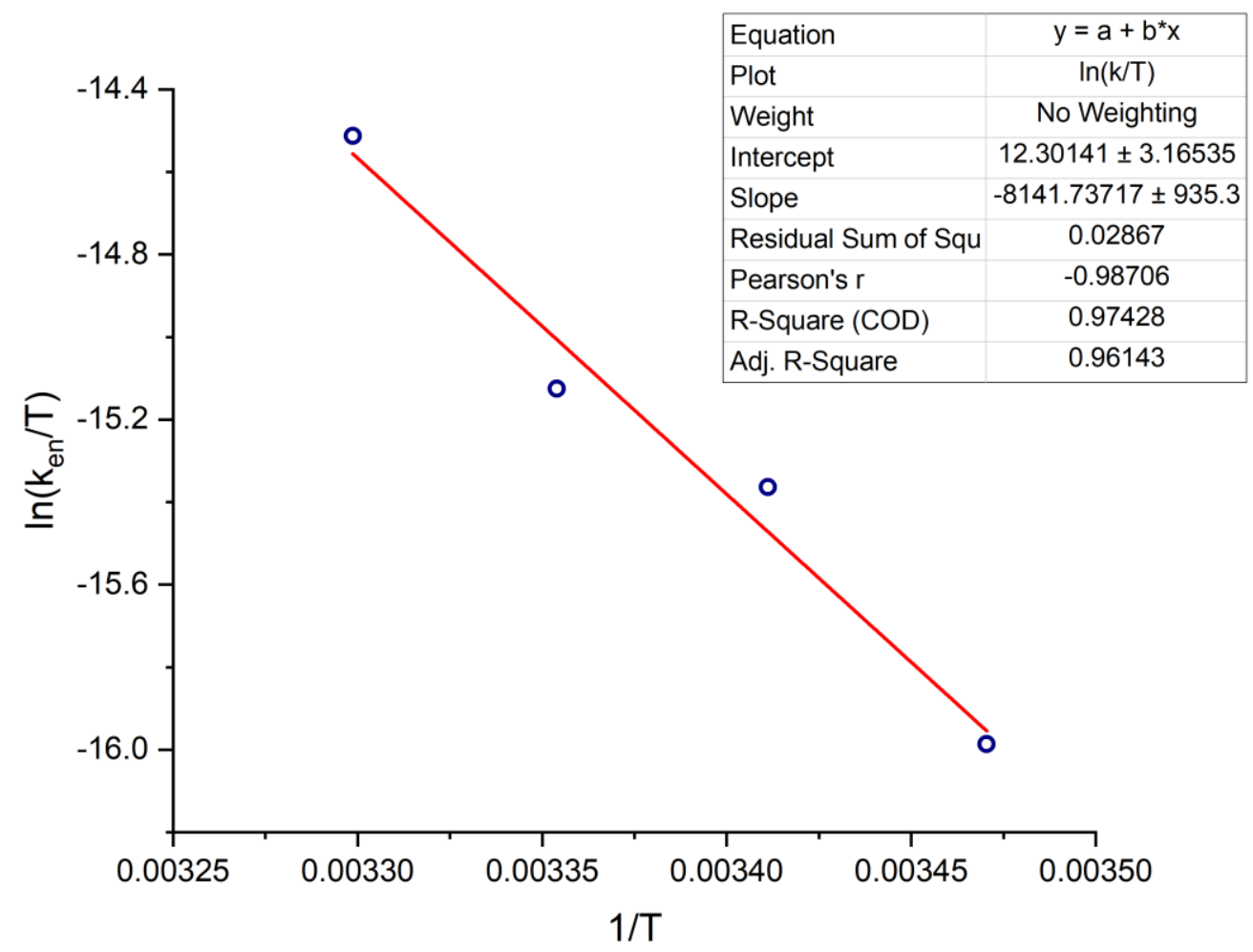

Figure S13: Eyring plot of compound 2a. 
With the Arrhenius equation $k_{e n}=A e^{-\frac{E a}{R T}}$ it is possible to plot $\ln \left(k_{e n}\right)$ vs. $\frac{1}{T}$ to get the activation energy:

$$
\ln \left(k_{\text {en }}\right)=-\left(\frac{E_{a}}{R}\right)\left(\frac{1}{T}\right)+\ln (A)
$$

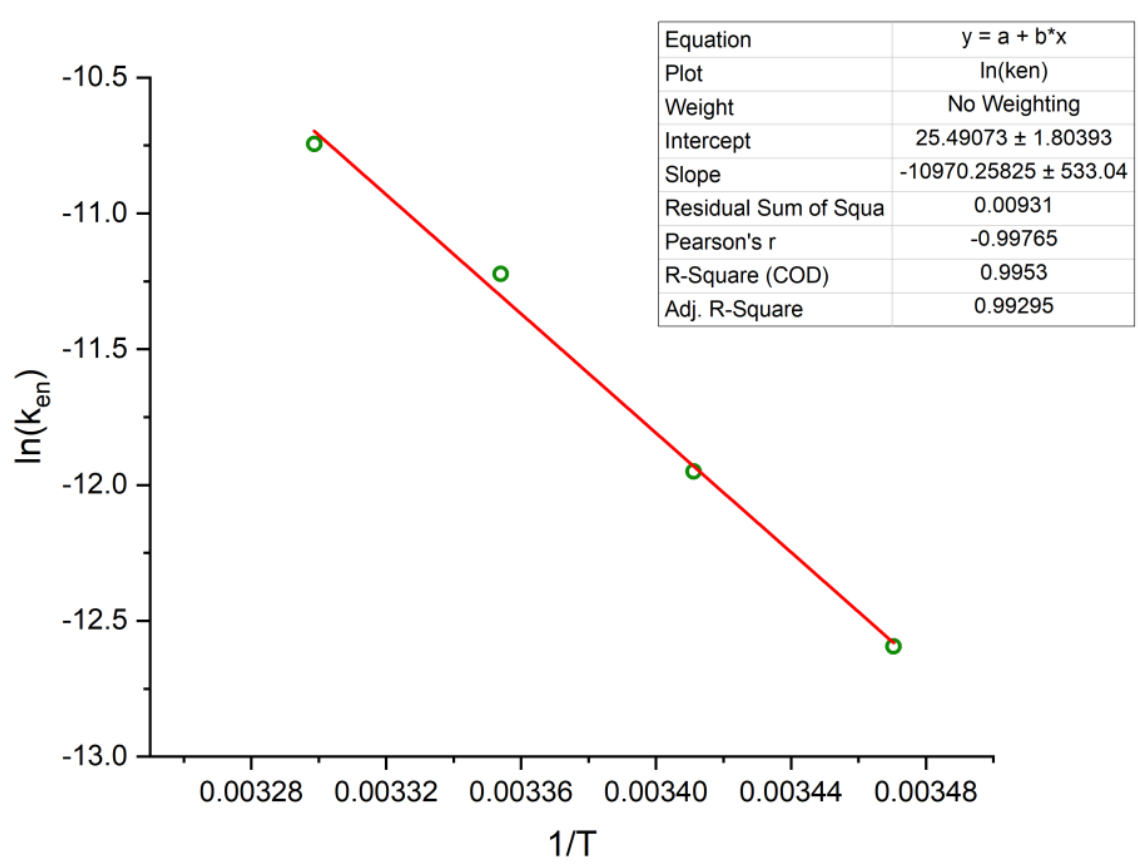

Figure S14: Arrhenius plot for compound 1a.

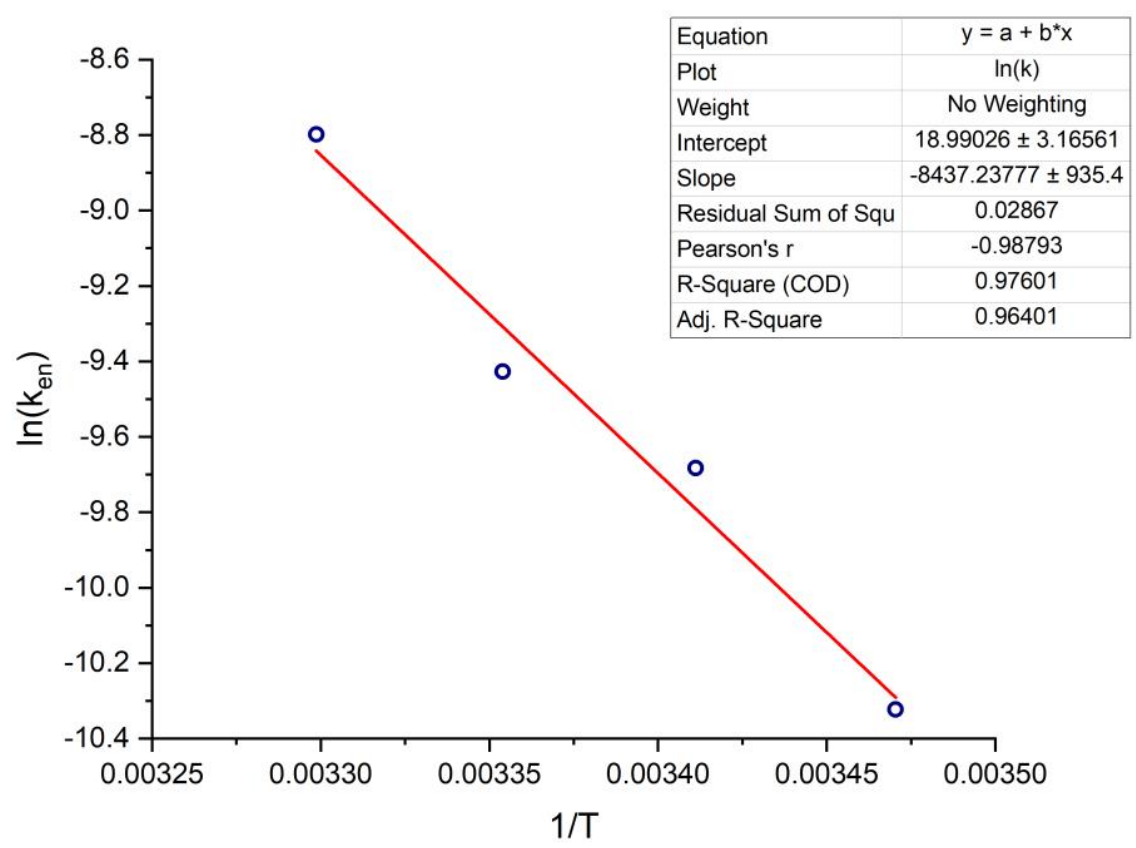

Figure S15: Arrhenius plot for compound 2a. 
Table S15: The obtained $E_{a}$ values for compound 1a and 2a.

\begin{tabular}{|l|r|r|}
\cline { 2 - 3 } \multicolumn{1}{c|}{} & Compound 1a & Compound 2a \\
\hline$E_{a}[\mathrm{~kJ} / \mathrm{mol}]$ & 91.2 & 70.2 \\
\hline
\end{tabular}




\section{Single Crystal X-Ray Analysis of 2a}

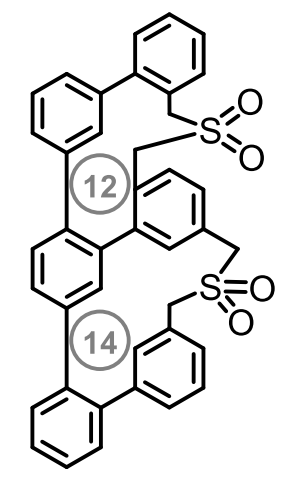

$2 a$

Crystals of $\mathbf{2 a}$ were grown from DCM by slow vapor diffusion technique with $n$-hexane at room temperature. formula $\mathrm{C}_{40} \mathrm{H}_{30} \mathrm{O}_{4} \mathrm{~S}_{2}, \mathrm{M}=638.80, \mathrm{~F}(000)=1576$, colourless plate, size $0.04 \times 0.2 \mathrm{x}$ $0.2 \mathrm{~mm}^{3}$, monoclinic, space group $P 12 / 1 / \mathrm{c} 1, \mathrm{Z}=4, \mathrm{a}=15.9695(11) \AA$, $b=17.6905(12) \AA$, $c=$ 14.3908(10) $\AA, \alpha=90^{\circ}, \beta=114.442(3)^{\circ}, y=90^{\circ}, V=3701.2(4) \AA^{3}$, Dcalc. $=1.332 \mathrm{Mg}^{*} \mathrm{~m}^{-3}$. The crystal was measured on a Bruker APEX-II CCD diffractometer at $130 \mathrm{~K}$ using graphitemonochromated $\mathrm{Cu}$ Ka-radiation with $\lambda=1.54178 \AA, \Theta \max =70.188^{\circ}$. Minimal $/ \mathrm{maximal}$ transmission $0.6474 / 0.7533, \mu=1.668 \mathrm{~mm}-1$. The Apex2 suite has been used for datacollection and integration. From a total of 24566 reflections, 6749 were independent (merging $r=0.0295$ ). From these, 6749 were considered as observed $(I>2 \sigma(I))$ and were used to refine 415 parameters. The structure was solved by dual methods using the program SHELXT 2014/5. Least-squares refinement against Fsqd was carried out on all non-hydrogen atoms using the program ShelXL. $R=0.0335$ (observed data), $w R=0.0988$ (all data), GOF $=1.065$. Minimal/maximal residual electron density $=-0.277 / 0.325$ e $\AA^{-3}$. Chebychev polynomial weights were used to complete the refinement. Plots were produced using Olex2. Crystallographic data (excluding structure factors) for the structure $\mathbf{2} \mathbf{a}$ in this paper have been deposited with the Cambridge Crystallographic Data Center, the deposition number is (1940617). Copies of the data can be obtained, free of charge, on application to the CCDC, 12 Union Road, Cambridge CB2 1EZ, UK [fax: +44-1223-336033 or e-mail: deposit@ccdc.cam.ac.uk]. 


\section{Determination of the torsion angles of the Geländer macrocycles}

All the data were abstracted from the XRD structure of $\mathbf{1 d}, \mathbf{1 b}, \mathbf{2} \mathbf{a}$ and $\mathbf{2 c}$ and from the geometry optimized structure of $\mathbf{1 a}, \mathbf{1} \mathbf{c}$ and $\mathbf{2} \mathbf{b}$. $^{1,3}$ The torsional angles of each biphenyl unit in the backbone of the helical system $\mathbf{1 a}$ and $\mathbf{2 a}$ were determined by measuring the two corresponding planes. The plane was defined by the mean of the six carbon atoms of each benzene unit (For $2 \mathrm{a}: 52.95^{\circ}$ and $71.06^{\circ}$; for $1 \mathrm{a}: 80.33^{\circ}$ and $73.24^{\circ}$; Scheme $\mathrm{S1}$ ).

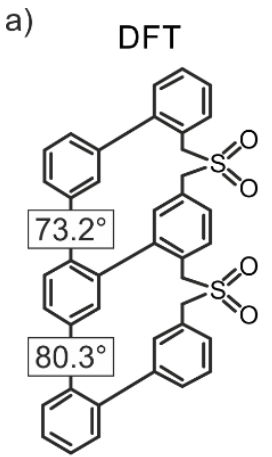

1a

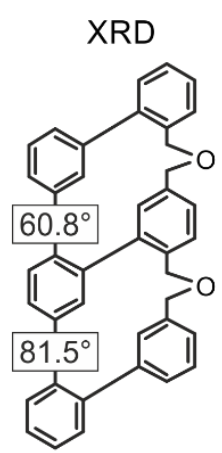

$1 d$

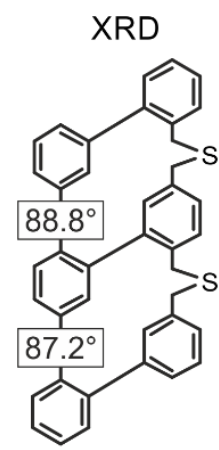

$1 b$

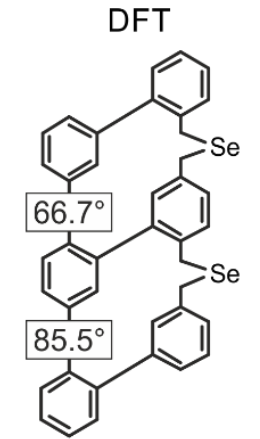

1c

$$
\Delta \mathrm{G}_{r a c}^{*}=97.4 \mathrm{~kJ} / \mathrm{mol} \quad \Delta \mathrm{G}_{r a c}^{*}=95.8 \mathrm{~kJ} / \mathrm{mol} \quad \Delta \mathrm{G}_{r a c}^{*}=93.5 \mathrm{~kJ} / \mathrm{mol} \quad \Delta \mathrm{G}_{r a c}^{*}=86.9 \mathrm{~kJ} / \mathrm{mol}
$$

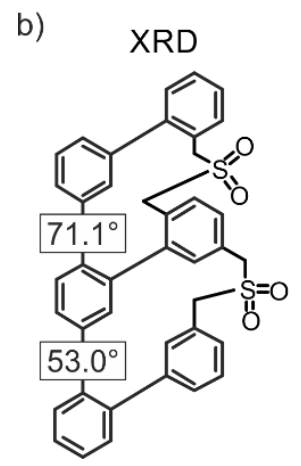

2a

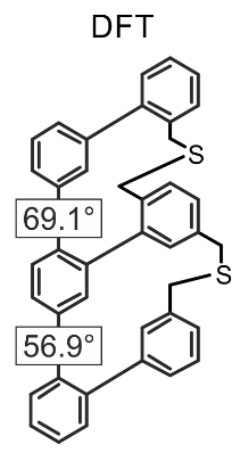

2b

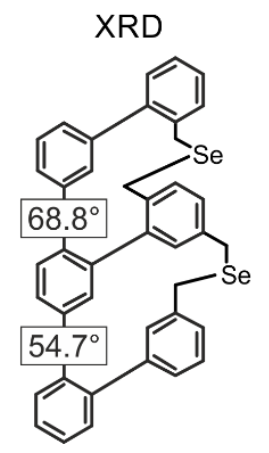

2c

$\Delta \mathrm{G}_{r a c}^{*}=92.3 \mathrm{~kJ} / \mathrm{mol}$

Scheme S1: The torsional angles of each biphenyl unit in the backbone of the helical system 1a-c and 2a-c. 


\section{Crystal packing of $2 a$}

The sample rac-2a was crystallized by slow evaporation of DCM and by slow diffusion of $n$ hexane (stepwise crystallization). The achiral unit cell comprising a pair of molecules of each enantiomer in a 2:2 ratio (Figure S16).

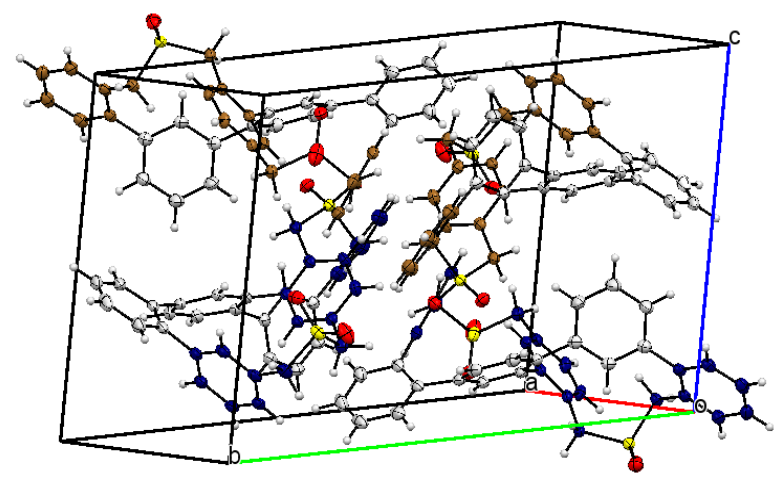

Figure S16: The unit cell of rac-2a (blue, brown), which contains two molecules of each enantiomer. Color code: Bridge = blue and brown, backbone $=$ gray, hydrogen atoms $=$ white, sulfur atoms $=$ yellow, oxygen atoms $=$ red .

\section{Calculated CD: Data}

Gaussian 09 (release D.01) program was used in all calculations. ${ }^{3}$ The molecular geometries were optimized using B3LYP functional and 6-31g(d) basis set with GD3BJ empirical dispersion (Figure S17). Stationary point was confirmed by subsequent frequency calculation. For the [12:14] sulfone Geländer $\mathbf{2 a}$, the single-crystal structure was used as the starting point.

The absolute configurations of respective enantiomers of [13:13] sulfone 1a and [12:14]-sulfone $\mathbf{2 a}$ were assigned by comparison of the calculated and measured CD spectra. The spectra were obtained using TD-CAM-B3LYP method with 6-31g(d) basis set calculating first 75 singlet and triplet excitations. The ECD spectra were compared using SpecDis (release 1.71). ${ }^{4}$ Empirical vibrational broadening of $0.2 \mathrm{eV}$ was used and the calculated spectra were shifted by $-1.25 \mathrm{eV}$. The velocity form of the rotatory strength was used. A good agreement of experimental and calculated data was observed. 
a)

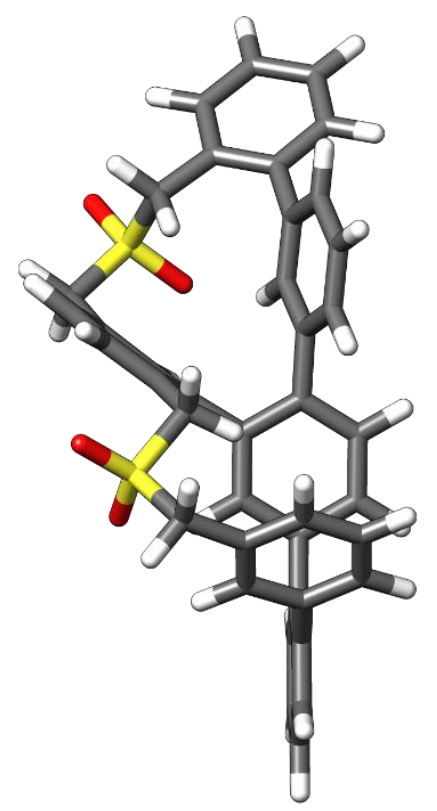

b)

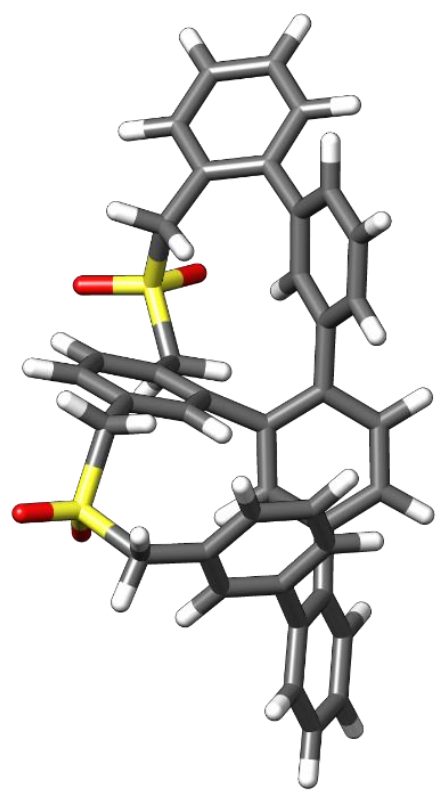

Figure S17: B3LYP-D3BJ/6-31 $\mathrm{g}^{\star}$ optimized structures of Geländers a) $M-1 \mathrm{a}$ and b) $M-2 \mathrm{a}$. 


\section{Computational Section}

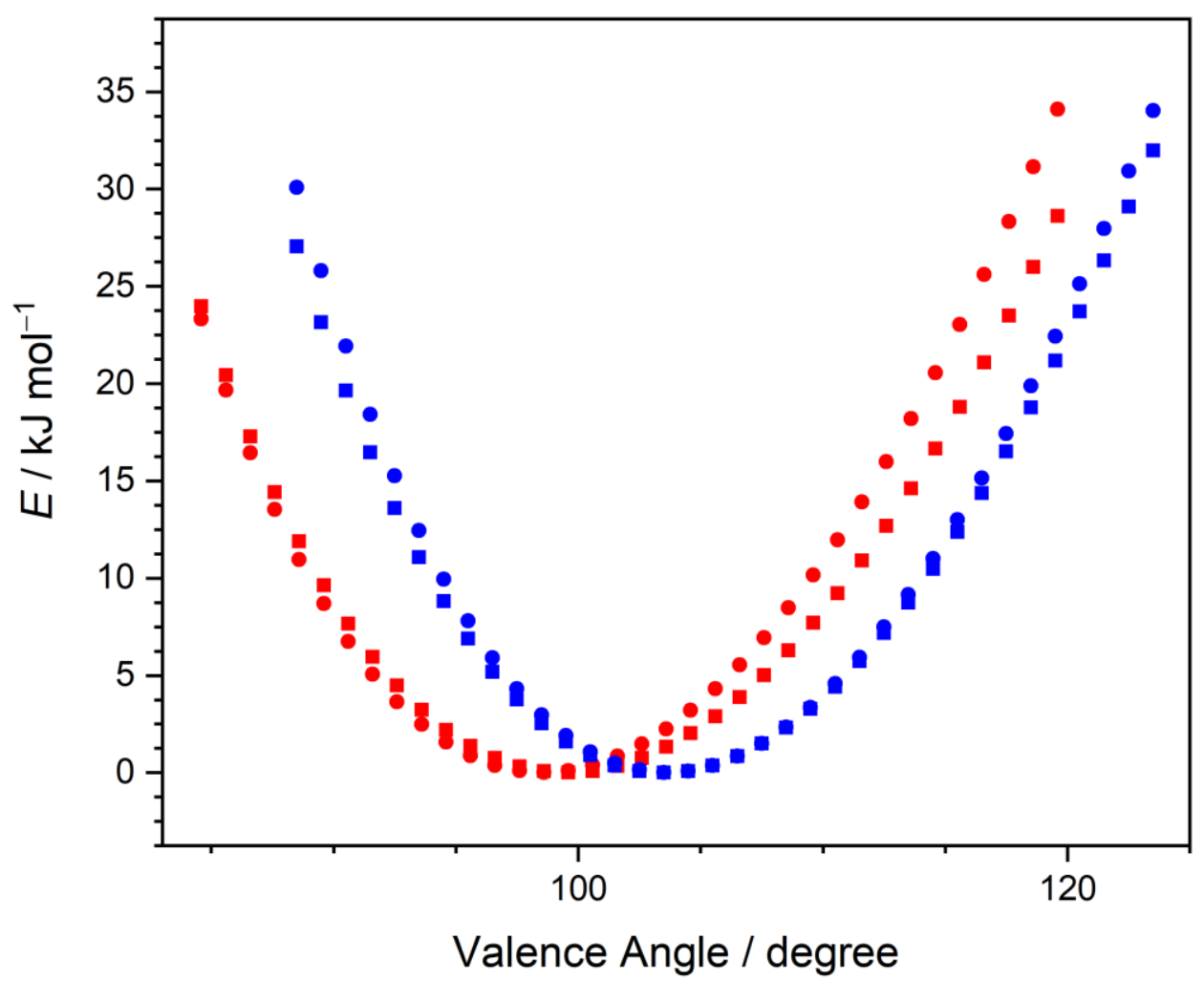

Figure S18. A relaxed potential energy surface for the $C-Y-C\left(Y=S, S_{2}\right)$ valence angle in dimethyl sulfide (red) and dimethyl sulfone (blue). The points were calculated in increments of 1.0 degree from the corresponding potential energy minima at B3LYP (squares) and MP2 (circles) levels of theory with 6-31G(d) basis set. 
Table S16. Torsion angles at biphenyl junctions ${ }^{a}$ in the terphenyl backbone calculated for $\mathbf{1} a, b$ and $\mathbf{2 a , b}$.

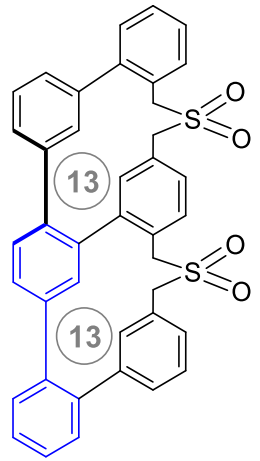

$1 \mathrm{a}$

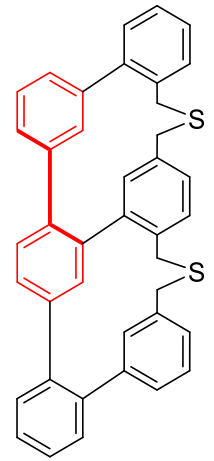

$1 \mathrm{~b}$<smiles>c1ccc(-c2ccccc2SCc2cccc(-c3cc(-c4ccccc4-c4ccccc4)ccc3-c3cccc(CSCc4ccccc4-c4ccccc4-c4ccccc4)c3)c2)cc1</smiles>

2b

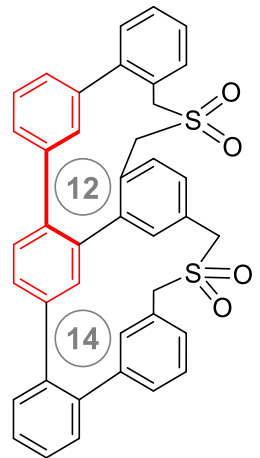

$2 a$

\begin{tabular}{|c|c|c|c|c|}
\hline Compound & Junction $^{a}$ & \multicolumn{2}{|c|}{ Torsion Angles ${ }^{b} /$ degrees } & Average / degrees \\
\hline \multirow[t]{6}{*}{$1 \mathrm{a}$} & 1 & -105.8 & -107.2 & -106.5 \\
\hline & & 69.9 & 77.1 & 73.5 \\
\hline & 2 & -98.3 & -101.7 & -100 \\
\hline & & 79.4 & 80.6 & 80 \\
\hline & SUM $^{c}$ & & & -206.5 \\
\hline & SUM $^{d}$ & & & 153.5 \\
\hline \multirow[t]{6}{*}{$1 b$} & 1 & -111.6 & -111.2 & -111.4 \\
\hline & & 65.3 & 71.9 & 68.6 \\
\hline & 2 & -91.2 & -95.5 & -93.35 \\
\hline & & 84.8 & 88.4 & 86.6 \\
\hline & SUM $^{c}$ & & & -204.75 \\
\hline & SUM $^{d}$ & & & 155.2 \\
\hline \multirow[t]{6}{*}{$2 a$} & 1 & -106.7 & -110.9 & -108.8 \\
\hline & & 68.4 & 74.1 & 71.25 \\
\hline & 2 & -122.9 & -122.4 & -122.65 \\
\hline & & 60 & 54.7 & 57.35 \\
\hline & SUM $^{c}$ & & & -231.45 \\
\hline & $\mathrm{SUM}^{d}$ & & & 128.6 \\
\hline \multirow[t]{6}{*}{$2 b$} & 1 & -108.6 & -112.2 & -110.4 \\
\hline & & 66.7 & 72.5 & 69.6 \\
\hline & 2 & -122.3 & -122 & -122.15 \\
\hline & & 60.6 & 55.1 & 57.85 \\
\hline & SUM $^{c}$ & & & -232.55 \\
\hline & $\mathrm{SUM}^{d}$ & & & 127.45 \\
\hline
\end{tabular}

a The biphenyl in the terphenyl backbone in each macrocyclic subunit of the Geländer helix. The junction 1 (highlighted in red) represents the 13- and 12-membered and junction 2 (highlighted in blue) represents the 13- and 14-membered macrocycle in 1 and 2, respectively. ${ }^{b}$ Four torsion angles in each junction exist (defined by the three bold bonds shown in junction 1 in the structures of $\mathbf{1} \mathbf{a}, \mathbf{b}$ and $\mathbf{2 a}, \mathbf{b}$ ). An average value is given for each pair. ${ }^{c}$ The sum of the average torsion angles from both junctions - the first pair of torsion angles. ${ }^{d}$ The sum of the average torsion angles from both junctions - the second pair of torsion angles. 
Table S17 The biphenyl torsion angles calculated for 5-7.

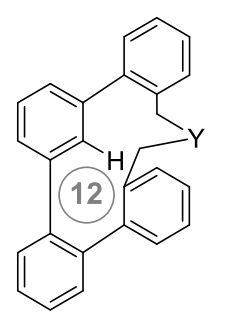

5a, $Y=\mathrm{SO}_{2}$

5b, $Y=S$

Compound

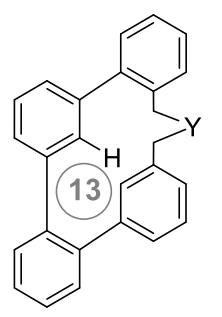

6a, $Y=\mathrm{SO}_{2}$

$6 b, Y=S$

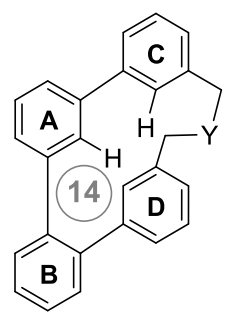

$7 \mathrm{a}, \mathrm{Y}=\mathrm{SO}_{2}$

$7 b, Y=S$

\begin{tabular}{cccc}
\hline $\mathbf{5 a}$ & -111.3 & -107.9 & -109.6 \\
& 70.6 & 70.2 & 70.4 \\
\hline $\mathbf{5 b}$ & -113 & -109 & -111 \\
& 68.8 & 69.2 & 69 \\
\hline $\mathbf{6 a}$ & -113 & -112.5 & -112.75 \\
& 64.8 & 69.7 & 67.25 \\
\hline $\mathrm{SUM}^{b}$ & & & -225.5 \\
$\mathrm{SUM}^{b}$ & & & 134.5 \\
\hline $\mathbf{6 b}$ & -112.4 & -112.5 & -112.45 \\
& 64.3 & 70.8 & 67.55 \\
\hline $\mathrm{SUM}^{b}$ & & & -224.9 \\
$\mathrm{SUM}^{b}$ & & & 135.1 \\
\hline $\mathbf{7 a}$ & -137.8 & -133.3 & -135.55 \\
& 45.1 & 43.8 & 44.45 \\
\hline $\mathrm{SUM}^{c}$ & & & -245.15 \\
$\mathrm{SUM}^{c}$ & & & 114.85 \\
\hline $\mathbf{7 b}^{b}$ & -136.8 & -132.9 & -134.85 \\
& 46 & 44.4 & 45.2 \\
\hline $\mathrm{SUM}^{c}$ & & & -245.85 \\
$\mathrm{SUM}^{c}$ & & & 114.2 \\
\hline
\end{tabular}

a See the caption to Table S20 for definition of the pairs and the average torsion angles. ${ }^{b}$ The sum of the two 13-membered macrocycles 6 as a model for one Geländer helix 1. ${ }^{c}$ The sum of the 12- and 14-membered macrocycles 5 and 7 as a model for one Geländer helix 2. 
Table S18. The biphenyl torsion angles in the transition states of the biphenyl backbone $(A-B)$ rotation calculated for 5-7.

\begin{tabular}{cccc} 
Compound & \multicolumn{2}{c}{ Torsion $_{\text {Angles }}$ / degrees } & Average / degrees \\
\hline $\mathbf{5 a}$ & -169.0 & -163.9 & -166.45 \\
& 13.1 & 14.0 & 13.55 \\
\hline $\mathbf{5 b}$ & 174.2 & -175.9 & 179.15 \\
& -1.7 & -0.0 & -0.85 \\
\hline $\mathbf{6 a}$ & -157.2 & -159.9 & -158.55 \\
& 20.1 & 22.7 & 21.4 \\
\hline $\mathbf{6 b}$ & -153.3 & -157.2 & -155.25 \\
& 23.1 & 26.4 & 24.75 \\
\hline $\mathbf{7 a}$ & 173.5 & 179.8 & 176.65 \\
& -3.5 & -3.1 & -3.3 \\
\hline $\mathbf{7 b}$ & 173.0 & 177.9 & 175.45 \\
& -4.8 & -4.3 & -4.55 \\
\hline
\end{tabular}

${ }^{a}$ See the caption to Table S20 for definition of the pairs and the average torsion angles. 
Table S19. The calculated energies ${ }^{a}$ of individual macrocyclic subunits of 1 and 2 cropped from the optimized geometries of energy minima of 1 and 2 with respect to fully optimized macrocycles 5-7.<smiles>[Y]c1ccccc1-c1cccc(-c2ccccc2-c2ccccc2)c1</smiles>

$6_{1}$<smiles>[Y]c1ccccc1-c1cccc(-c2ccccc2-c2ccccc2)c1</smiles>

62<smiles>[Y]Cc1cccc(Cc2ccccc2-c2ccccc2-c2cccc(-c3ccc(CCc4ccccc4-c4ccccc4)cc3-c3ccccc3)c2)c1</smiles>

1a, $\mathrm{Y}=\mathrm{SO}_{2}$

1b, $Y=S$<smiles>[Y]Cc1cccc(-c2cc(-c3cccc(-c4ccccc4C[Y])c3)ccc2-c2ccccc2-c2ccccc2-c2ccccc2)c1</smiles>

2a, $Y=\mathrm{SO}_{2}$

2b, $Y=S$<smiles>[X]c1ccccc1-c1cccc(-c2ccccc2-c2ccccc2)c1</smiles><smiles>c1ccc(CCc2cccc(-c3ccccc3-c3cccc(-c4ccccc4)c3)c2)cc1</smiles>

$7_{1}$

\begin{tabular}{cccc}
\hline \multirow{2}{*}{ Molecule } & \multicolumn{3}{c}{ Energy $/ \mathrm{kJ} \mathrm{mol}^{-1}$} \\
\cline { 2 - 4 } & B3LYP & BMK & M06-2X \\
\hline $\mathbf{6}_{\mathbf{1}} \mathbf{a}$ & 2.8 & 2.6 & 3.6 \\
$\mathbf{6}_{\mathbf{2}} \mathbf{a}$ & 5.6 & 7.3 & 8.1 \\
$\mathbf{\mathbf { } _ { 1 } \mathbf { b }}$ & 2.5 & 2.1 & 2.7 \\
$\mathbf{\mathbf { 6 } _ { 2 } \mathbf { b }}$ & 4.3 & 6.7 & 6.9 \\
$\mathbf{5}_{\mathbf{1}} \mathbf{a}$ & 3.0 & 3.2 & 3.5 \\
$\mathbf{7}_{\mathbf{1}} \mathbf{a}$ & 18.5 & 20.3 & 21.8 \\
$\mathbf{5}_{\mathbf{b}} \mathbf{b}$ & 11.4 & 12.7 & 14.6 \\
$\mathbf{\mathbf { 7 } _ { 1 } \mathbf { b }}$ & 20.0 & 20.7 & 23.3 \\
\hline
\end{tabular}

a The structures were cropped from the minimum energy geometries of $\mathbf{1}$ and $\mathbf{2}$. The two $\mathrm{C}-\mathrm{C}$ disconnections were capped by the corresponding two $\mathrm{C}-\mathrm{H}$ bonds. The coordinates of the two added hydrogen atoms were fully optimized while the remaining coordinates of all the other atoms were kept frozen/identical to those calculated for the corresponding macrocyclic subunit in the Geländer helices 1 and 2. Only electronic energies (gas phase, $0 \mathrm{~K}$ ) without the ZPVE correction are, therefore, reported because the obtained structures of the macrocyclic subunits do not represent the potential energy minima as those found for 5-7 in a fully relaxed geometry optimization process. 


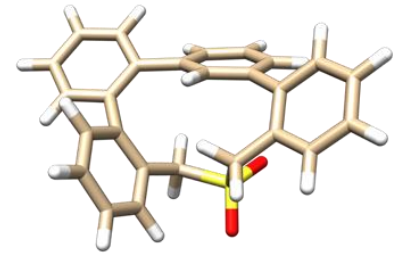

5a-intermediate $40.9 \mathrm{kcal} \mathrm{mol}^{-1}$

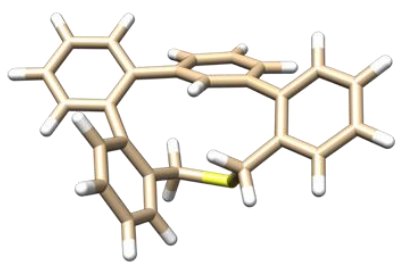

5b-intermediate $32.4 \mathrm{kcal} \mathrm{mol}^{-1}$

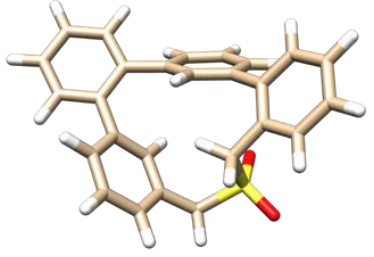

6a-intermediate $14.2 \mathrm{kcal} \mathrm{mol}^{-1}$

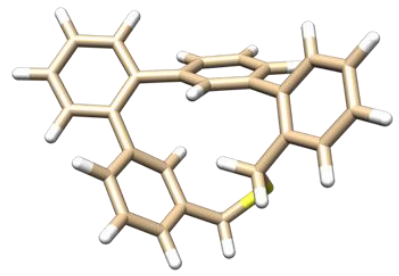

6b-intermediate $6.8 \mathrm{kcal} \mathrm{mol}^{-1}$

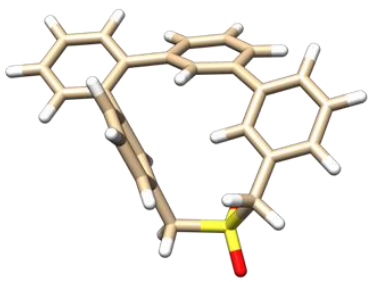

7a-intermediate $37.8 \mathrm{kcal} \mathrm{mol}^{-1}$

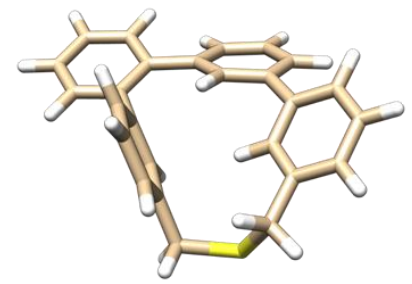

7b-intermediate $29.8 \mathrm{kcal} \mathrm{mol}^{-1}$

Figure S19. The structure of the intermediates reached after the rate-limiting rotation of the biphenyl backbone in 5-7. The energies are the energies of formation from 5-7 at the M06-2X/cc-pVTZ level of theory on B3LYP/6-31G(d) geometries. See Table S20 for additional details about their energies.

Table S20. The calculated reaction free energies ${ }^{a}$ of the biphenyl backbone rotation in 5-7.

\begin{tabular}{cccc}
\hline \multirow{2}{*}{ Molecule } & \multicolumn{3}{c}{ Energy / kJ mol ${ }^{-1}$} \\
\cline { 2 - 4 } & B3LYP & BMK & M06-2X \\
\hline $\mathbf{5 a}$ & 49.3 & 44.6 & 40.9 \\
$\mathbf{6 a}$ & 18.2 & 17.4 & 14.2 \\
$\mathbf{7 a}$ & 33.8 & 36.4 & 37.8 \\
\hline $\mathbf{5 b}$ & 40.8 & 35.4 & 32.4 \\
$\mathbf{6 b}$ & 14.3 & 10.0 & 6.8 \\
$\mathbf{7 b}$ & 26.8 & 28.6 & 29.8 \\
\hline
\end{tabular}

${ }^{a}$ Energies (gas phase, $E=E_{\mathrm{el}}+E_{\text {corr }}, 298.15 \mathrm{~K}$ ) calculated with the corresponding functional and cc-pVTZ basis set on B3LYP/6$31 \mathrm{G}(\mathrm{d})$ optimized geometries. Thermal corrections to free energy were not scaled and the low-frequency vibrations were treated explicitly and not as free rotors. 
Table S21. The calculated activation energies a of biphenyl $(A-B)$ backbone rotation in 5-7.

\begin{tabular}{cccc}
\hline \multirow{2}{*}{ Molecule } & \multicolumn{3}{c}{ Energy / kJ mol ${ }^{-1}$} \\
\cline { 2 - 4 } & B3LYP & BMK & M06-2X \\
\hline 5a & 72.6 & 72.9 & 73.0 \\
$\mathbf{6 a}$ & 86.1 & 88.2 & 88.7 \\
$\mathbf{7 a}$ & $37.8\left(20.2^{b}\right)$ & $40.4\left(21.4^{b}\right)$ & $43.3\left(20.9^{b}\right)$ \\
\hline $\mathbf{5 b}$ & 57.8 & 59.5 & 59.2 \\
$\mathbf{6 b}$ & 81.3 & 81.2 & 80.9 \\
$\mathbf{7 b}$ & $28.9\left(14.1^{b}\right)$ & $31.7\left(16.8^{b}\right)$ & $33.6\left(17.0^{b}\right)$ \\
\hline
\end{tabular}

${ }^{a}$ Energies (gas phase, $E=E_{\mathrm{el}}+E_{\mathrm{ZPVE}}, 0 \mathrm{~K}$ ) calculated with the corresponding functional and cc-pVTZ basis set on B3LYP/6-31G(d) optimized geometries. Zero-point vibrational energy (ZPVE) corrections were not scaled. ${ }^{b}$ Rotation of the A-C biphenyl unit.

Table S22. The calculated activation free energies ${ }^{a}$ of biphenyl $(A-B)$ backbone rotation in 5-7.

\begin{tabular}{cccc}
\hline \multirow{2}{*}{ Molecule } & \multicolumn{3}{c}{ Energy / kJ mol } \\
\cline { 2 - 4 } & B3LYP & BMK & M06-2X \\
\hline $\mathbf{5 a}$ & 79.5 & 79.7 & 79.8 \\
$\mathbf{6 a}$ & 92.3 & 94.4 & 94.9 \\
$\mathbf{7 a}$ & 40.2 & 42.9 & 45.8 \\
\hline $\mathbf{5 b}$ & 61.2 & 63.0 & 62.7 \\
$\mathbf{6 b}$ & 89.3 & 89.3 & 88.9 \\
$\mathbf{7 b}$ & 32.2 & 35.0 & 36.9 \\
\hline
\end{tabular}

a Energies (gas phase, $E=E_{\mathrm{el}}+E_{\text {corr }}, 298.15 \mathrm{~K}$ ) calculated with the corresponding functional and cc-pVTZ basis set on B3LYP/6$31 \mathrm{G}(\mathrm{d})$ optimized geometries. Thermal corrections to free energy were not scaled and the low-frequency vibrations were treated explicitly and not as free rotors.

Table S23. The calculated activation enthalpies ${ }^{a}$ of biphenyl $(A-B)$ backbone rotation in 5-7.

\begin{tabular}{cccc}
\hline \multirow{2}{*}{ Molecule } & \multicolumn{3}{c}{ Energy $/ \mathrm{kJ} \mathrm{mol}^{-1}$} \\
\cline { 2 - 4 } & B3LYP & BMK & M06-2X \\
\hline $\mathbf{5 a}$ & 69.8 & 70.0 & 70.1 \\
$\mathbf{6 a}$ & 83.4 & 85.5 & 86.0 \\
$\mathbf{7 a}$ & 35.9 & 38.6 & 41.5 \\
\hline $\mathbf{5 b}$ & 55.6 & 57.4 & 57.0 \\
$\mathbf{6 b}$ & 78.1 & 78.0 & 77.7 \\
$\mathbf{7 b}$ & 26.9 & 29.7 & 31.6
\end{tabular}

${ }^{a}$ Energies (gas phase, $E=E_{\mathrm{el}}+E_{\mathrm{corr}}, 298.15 \mathrm{~K}$ ) calculated with the corresponding functional and cc-pVTZ basis set on B3LYP/6$31 \mathrm{G}(\mathrm{d})$ optimized geometries. Thermal corrections to enthalpies were not scaled and the low-frequency vibrations were treated explicitly and not as free rotors. 


\section{Cartesian Coordinates}

\section{Atomic coordinates for ECD calculations.}

M-1a, $E_{\text {el }}(B 3 L Y P-D 3 B J / 6-31 G(d)$, superfine integration grid $)=-2639.75170408$ Hartrees

$\begin{array}{rrrr}\text { S } & 4.30375 & 0.16682 & -2.21304 \\ \mathrm{~S} & -2.51142 & 3.14967 & 0.08082 \\ \mathrm{O} & 5.55143 & 0.28178 & -2.98824 \\ \mathrm{O} & 3.60626 & -1.13019 & -2.13002 \\ \mathrm{O} & -2.82347 & 2.51479 & -1.21199 \\ \mathrm{O} & -2.10986 & 4.56701 & 0.12791 \\ \mathrm{C} & 1.08564 & 0.57120 & -1.68095 \\ \mathrm{C} & 0.08590 & 0.70620 & -0.71820 \\ \mathrm{C} & -0.20726 & -1.34688 & 0.68293 \\ \mathrm{C} & -0.69793 & -0.50477 & -0.33568 \\ \mathrm{C} & 2.30310 & -1.08665 & 0.98890 \\ \mathrm{H} & 2.47184 & -1.43010 & -0.02669 \\ \mathrm{C} & -1.91162 & -0.80414 & -0.96192 \\ \mathrm{H} & -2.30479 & -0.11274 & -1.70004 \\ \mathrm{C} & 0.76531 & 3.00894 & -0.39137 \\ \mathrm{C} & 4.81225 & -0.97330 & 1.34127 \\ \mathrm{C} & 5.39074 & -0.25680 & 0.27468 \\ \mathrm{C} & -0.08273 & 1.94289 & -0.07028 \\ \mathrm{C} & -4.33785 & 1.52898 & 1.42967 \\ \mathrm{C} & 3.40598 & -0.78524 & 1.80230 \\ \mathrm{C} & -0.93276 & -2.49805 & 1.01131 \\ \mathrm{H} & -0.55345 & -3.15290 & 1.79044 \\ \mathrm{C} & 1.78714 & 2.84824 & -1.32163 \\ \mathrm{H} & 2.45655 & 3.67640 & -1.53875 \\ \mathrm{C} & 1.95503 & 1.62450 & -1.97826 \\ \mathrm{C} & -1.20431 & 2.15171 & 0.90392 \\ \mathrm{C} & -4.82014 & -1.18940 & 1.88040 \\ \mathrm{H} & -4.98274 & -2.24996 & 2.04647 \\ \mathrm{C} & 0.99716 & -0.98953 & 1.48980 \\ \mathrm{C} & -2.64475 & -1.94293 & -0.60984 \\ \mathrm{C} & 4.61087 & 0.76315 & -0.50590 \\ \mathrm{H} & 3.63583 & 0.96945 & -0.06486 \\ \mathrm{H} & 5.16077 & 1.70099 & -0.62922 \\ \mathrm{C} & -2.12902 & -2.80028 & 0.36710 \\ \mathrm{H} & -2.68816 & -3.68896 & 0.64542 \\ \mathrm{C} & -4.23071 & 0.99800 & 2.72001 \\ \mathrm{H} & -3.94374 & 1.64354 & 3.54644 \\ \mathrm{C} & -4.91628 & -0.67589 & 0.58108 \\ \mathrm{C} & -4.49014 & -0.35358 & 2.94583 \\ \mathrm{H} & -4.41470 & -0.75885 & 3.95073 \\ \mathrm{C} & 5.61543 & -1.87973 & 2.05120 \\ \mathrm{H} & 5.17123 & -2.42945 & 2.87569 \\ \mathrm{C} & 6.73246 & -0.47932 & -0.06411 \\ \mathrm{H} & -16045 & 0.06994 & -0.89663 \\ \mathrm{C} & -4.70142 & 0.68751 & 0.37162 \\ \mathrm{H} & -4.74920 & 1.08537 & -0.63555 \\ \mathrm{C} & -5.12340 & -1.57850 & -0.58909 \\ \mathrm{C} & -4.00008 & -2.19166 & -1.18328 \\ \mathrm{C} & -50757 & -1.39598 & 0.63890 \\ \mathrm{C} & & -1.55842 & 0.35689\end{array}$




$\begin{array}{rrrr}\text { C } & 6.94580 & -2.09791 & 1.70541 \\ \text { H } & 7.54000 & -2.81430 & 2.26551 \\ \mathrm{C} & 3.11992 & 1.39961 & -2.89503 \\ \mathrm{C} & 3.18370 & -0.36434 & 3.12280 \\ \mathrm{H} & 4.03635 & -0.12352 & 3.75103 \\ \mathrm{C} & -3.98167 & 2.96317 & 1.16773 \\ \mathrm{H} & -4.75145 & 3.50833 & 0.61098 \\ \mathrm{H} & -3.75598 & 3.51692 & 2.08248 \\ \mathrm{C} & 0.80119 & -0.57210 & 2.81316 \\ \mathrm{H} & -0.21215 & -0.49884 & 3.19873 \\ \mathrm{C} & -4.18197 & -3.03273 & -2.28508 \\ \mathrm{H} & -3.31357 & -3.50195 & -2.73857 \\ \mathrm{C} & -5.45708 & -3.26465 & -2.80161 \\ \mathrm{H} & -5.58237 & -3.91849 & -3.65996 \\ \mathrm{C} & -6.39655 & -1.81530 & -1.11483 \\ \mathrm{H} & -7.25600 & -1.33692 & -0.65359 \\ \mathrm{C} & -6.56604 & -2.65475 & -2.21621 \\ \mathrm{H} & -7.56108 & -2.83039 & -2.61496 \\ \mathrm{C} & 1.88937 & -0.25259 & 3.62303 \\ \mathrm{H} & 1.72875 & 0.07784 & 4.64557 \\ \mathrm{H} & -0.90651 & 2.72595 & 1.78491 \\ \mathrm{H} & -1.66808 & 1.21288 & 1.20933 \\ \mathrm{H} & 3.69708 & 2.30871 & -3.07959 \\ \mathrm{H} & 2.84077 & 0.95651 & -3.85670 \\ \mathrm{H} & 1.22764 & -0.39035 & -2.16066 \\ \mathrm{H} & 0.61594 & 3.96974 & 0.09087 \\ & & & \end{array}$

$\boldsymbol{P}-1 \mathrm{a}, E_{\text {el }}(\mathrm{B} 3 \mathrm{LYP}-\mathrm{D} 3 \mathrm{BJ} / 6-31 \mathrm{G}(\mathrm{d})$, superfine integration grid $)=-2639.75170408$ Hartrees

$\begin{array}{rrrr}\text { S } & -4.30375 & 0.16682 & -2.21304 \\ \text { S } & 2.51142 & 3.14967 & 0.08082 \\ \mathrm{O} & -5.55143 & 0.28178 & -2.98824 \\ \mathrm{O} & -3.60626 & -1.13019 & -2.13002 \\ \mathrm{O} & 2.82347 & 2.51479 & -1.21199 \\ \mathrm{O} & 2.10986 & 4.56701 & 0.12791 \\ \mathrm{C} & -1.08564 & 0.57120 & -1.68095 \\ \mathrm{C} & -0.08590 & 0.70620 & -0.71820 \\ \mathrm{C} & 0.20726 & -1.34688 & 0.68293 \\ \mathrm{C} & 0.69793 & -0.50477 & -0.33568 \\ \mathrm{C} & -2.30310 & -1.08665 & 0.98890 \\ \mathrm{H} & -2.47184 & -1.43010 & -0.02669 \\ \mathrm{C} & 1.91162 & -0.80414 & -0.96192 \\ \mathrm{H} & 2.30479 & -0.11274 & -1.70004 \\ \mathrm{C} & -0.76531 & 3.00894 & -0.39137 \\ \mathrm{C} & -4.81225 & -0.97330 & 1.34127 \\ \mathrm{C} & -5.39074 & -0.25680 & 0.27468 \\ \mathrm{C} & 0.08273 & 1.94289 & -0.07028 \\ \mathrm{C} & 4.33785 & 1.52898 & 1.42967 \\ \mathrm{C} & -3.40598 & -0.78524 & 1.80230 \\ \mathrm{C} & 0.93276 & -2.49805 & 1.01131 \\ \mathrm{H} & 0.55345 & -3.15290 & 1.79044 \\ \mathrm{C} & -1.78714 & 2.84824 & -1.32163 \\ \mathrm{H} & -2.45655 & 3.67640 & -1.53875 \\ \mathrm{C} & -1.95503 & 1.62450 & -1.97826 \\ \mathrm{C} & 1.20431 & 2.15171 & 0.90392 \\ \mathrm{C} & 4.82014 & -1.18940 & 1.88040 \\ \mathrm{H} & 4.98274 & -2.24996 & 2.04647 \\ \mathrm{C} & -0.99716 & -0.98953 & 1.48980\end{array}$




\begin{tabular}{|c|c|c|c|}
\hline C & 2.64475 & -1.94293 & -0.60984 \\
\hline C & -4.61087 & 0.76315 & -0.50590 \\
\hline $\mathrm{H}$ & -3.63583 & 0.96945 & -0.06486 \\
\hline $\mathrm{H}$ & -5.16077 & 1.70099 & -0.62922 \\
\hline C & 2.12902 & -2.80028 & 0.36710 \\
\hline $\mathrm{H}$ & 2.68816 & -3.68896 & 0.64542 \\
\hline $\mathrm{C}$ & 4.23071 & 0.99800 & 2.72001 \\
\hline $\mathrm{H}$ & 3.94374 & 1.64354 & 3.54644 \\
\hline $\mathrm{C}$ & 4.91628 & -0.67589 & 0.58108 \\
\hline C & 4.49014 & -0.35358 & 2.94583 \\
\hline $\mathrm{H}$ & 4.41470 & -0.75885 & 3.95073 \\
\hline $\mathrm{C}$ & -5.61543 & -1.87973 & 2.05120 \\
\hline $\mathrm{H}$ & -5.17123 & -2.42945 & 2.87569 \\
\hline C & -6.73246 & -0.47932 & -0.06411 \\
\hline $\mathrm{H}$ & -7.16045 & 0.06994 & -0.89663 \\
\hline $\mathrm{C}$ & 4.70142 & 0.68751 & 0.37162 \\
\hline $\mathrm{H}$ & 4.74920 & 1.08537 & -0.63555 \\
\hline C & 5.12340 & -1.57850 & -0.58909 \\
\hline C & 4.00008 & -2.19166 & -1.18328 \\
\hline C & -7.50757 & -1.39598 & 0.63890 \\
\hline $\mathrm{H}$ & -8.54356 & -1.55842 & 0.35689 \\
\hline C & -6.94580 & -2.09791 & 1.70541 \\
\hline $\mathrm{H}$ & -7.54000 & -2.81430 & 2.26551 \\
\hline C & -3.11992 & 1.39961 & -2.89503 \\
\hline C & -3.18370 & -0.36434 & 3.12280 \\
\hline $\mathrm{H}$ & -4.03635 & -0.12352 & 3.75103 \\
\hline C & 3.98167 & 2.96317 & 1.16773 \\
\hline $\mathrm{H}$ & 4.75145 & 3.50833 & 0.61098 \\
\hline $\mathrm{H}$ & 3.75598 & 3.51692 & 2.08248 \\
\hline C & -0.80119 & -0.57210 & 2.81316 \\
\hline $\mathrm{H}$ & 0.21215 & -0.49884 & 3.19873 \\
\hline C & 4.18197 & -3.03273 & -2.28508 \\
\hline $\mathrm{H}$ & 3.31357 & -3.50195 & -2.73857 \\
\hline C & 5.45708 & -3.26465 & -2.80161 \\
\hline $\mathrm{H}$ & 5.58237 & -3.91849 & -3.65996 \\
\hline C & 6.39655 & -1.81530 & -1.11483 \\
\hline $\mathrm{H}$ & 7.25600 & -1.33692 & -0.65359 \\
\hline C & 6.56604 & -2.65475 & -2.21621 \\
\hline $\mathrm{H}$ & 7.56108 & -2.83039 & -2.61496 \\
\hline C & -1.88937 & -0.25259 & 3.62303 \\
\hline $\mathrm{H}$ & -1.72875 & 0.07784 & 4.64557 \\
\hline $\mathrm{H}$ & 0.90651 & 2.72595 & 1.78491 \\
\hline $\mathrm{H}$ & 1.66808 & 1.21288 & 1.20933 \\
\hline $\mathrm{H}$ & -3.69708 & 2.30871 & -3.07959 \\
\hline $\mathrm{H}$ & -2.84077 & 0.95651 & -3.85670 \\
\hline $\mathrm{H}$ & -1.22764 & -0.39035 & -2.16066 \\
\hline $\mathrm{H}$ & -0.61594 & 3.96974 & 0.09087 \\
\hline
\end{tabular}

M-2a, $E_{\text {el }}(B 3 L Y P-D 3 B J / 6-31 G(d)$, superfine integration grid) $=-2639.73927763$ Hartrees

$\begin{array}{rrrr}S & 3.51299 & 0.46466 & -2.25649 \\ S & -3.30666 & 3.65306 & 0.02992 \\ O & 3.63474 & 1.58815 & -3.20709 \\ O & 4.18411 & -0.81707 & -2.52966 \\ O & -3.16207 & 2.88401 & -1.21545 \\ O & -3.69672 & 5.07359 & -0.00331 \\ C & 0.92784 & 0.98123 & -1.20436\end{array}$




\begin{tabular}{|c|c|c|c|}
\hline C & 0.07263 & 0.44094 & -0.22641 \\
\hline C & 0.44274 & -1.95990 & 0.66945 \\
\hline $\mathrm{C}$ & -0.25724 & -1.01463 & -0.10531 \\
\hline C & 2.87553 & -1.31173 & 0.85552 \\
\hline $\mathrm{H}$ & 2.99701 & -1.45930 & -0.21099 \\
\hline $\mathrm{C}$ & -1.47719 & -1.37873 & -0.68066 \\
\hline $\mathrm{H}$ & -2.03276 & -0.62547 & -1.22927 \\
\hline $\mathrm{C}$ & -0.71150 & 2.68440 & 0.32565 \\
\hline $\mathrm{C}$ & 5.25658 & -0.52739 & 0.93855 \\
\hline C & 5.30211 & 0.41039 & -0.11462 \\
\hline C & -0.72453 & 1.30631 & 0.53033 \\
\hline $\mathrm{H}$ & -1.41710 & 0.87560 & 1.24567 \\
\hline $\mathrm{C}$ & -4.29369 & 1.35019 & 1.32116 \\
\hline $\mathrm{C}$ & 3.98174 & -0.89021 & 1.60578 \\
\hline $\mathrm{C}$ & -0.08741 & -3.25364 & 0.78838 \\
\hline $\mathrm{H}$ & 0.45791 & -3.98531 & 1.37751 \\
\hline $\mathrm{C}$ & 0.16041 & 3.21511 & -0.63122 \\
\hline $\mathrm{H}$ & 0.17594 & 4.28552 & -0.81681 \\
\hline C & 0.97334 & 2.37244 & -1.37994 \\
\hline $\mathrm{H}$ & 1.62920 & 2.78570 & -2.14024 \\
\hline $\mathrm{C}$ & -1.71868 & 3.55059 & 1.01080 \\
\hline $\mathrm{H}$ & -1.99302 & 3.17991 & 2.00196 \\
\hline $\mathrm{H}$ & -1.41097 & 4.59557 & 1.08761 \\
\hline C & -3.69849 & -1.34154 & 1.79040 \\
\hline $\mathrm{H}$ & -3.46412 & -2.38531 & 1.96962 \\
\hline C & 1.64661 & -1.58342 & 1.46085 \\
\hline C & -2.04501 & -2.64030 & -0.49172 \\
\hline C & 4.07717 & 1.12079 & -0.60794 \\
\hline $\mathrm{H}$ & 3.23342 & 1.05150 & 0.07796 \\
\hline $\mathrm{H}$ & 4.27485 & 2.17259 & -0.82252 \\
\hline C & -1.31122 & -3.59761 & 0.21711 \\
\hline $\mathrm{H}$ & -1.72577 & -4.58986 & 0.37281 \\
\hline C & -3.78679 & 0.94472 & 2.56019 \\
\hline $\mathrm{H}$ & -3.63188 & 1.67959 & 3.34666 \\
\hline $\mathrm{C}$ & -4.21606 & -0.95947 & 0.54143 \\
\hline C & -3.48889 & -0.39903 & 2.79137 \\
\hline $\mathrm{H}$ & -3.10031 & -0.71051 & 3.75661 \\
\hline $\mathrm{C}$ & 6.45592 & -1.10028 & 1.38639 \\
\hline $\mathrm{H}$ & 6.41777 & -1.82265 & 2.19655 \\
\hline $\mathrm{C}$ & 6.53409 & 0.72310 & -0.70170 \\
\hline $\mathrm{H}$ & 6.55953 & 1.44350 & -1.51510 \\
\hline C & -4.50968 & 0.39058 & 0.32156 \\
\hline $\mathrm{H}$ & -4.87468 & 0.70743 & -0.65039 \\
\hline $\mathrm{C}$ & -4.46494 & -1.99027 & -0.50802 \\
\hline $\mathrm{C}$ & -3.44030 & -2.86296 & -0.94808 \\
\hline $\mathrm{C}$ & 7.71349 & 0.13132 & -0.26126 \\
\hline $\mathrm{H}$ & 8.65726 & 0.38386 & -0.73516 \\
\hline $\mathrm{C}$ & 7.67395 & -0.78223 & 0.79290 \\
\hline $\mathrm{H}$ & 8.58785 & -1.24947 & 1.14824 \\
\hline C & 1.71871 & 0.07929 & -2.10745 \\
\hline $\mathrm{H}$ & 1.64621 & -0.96543 & -1.80962 \\
\hline $\mathrm{H}$ & 1.39569 & 0.16254 & -3.15123 \\
\hline C & 3.85638 & -0.79985 & 2.99902 \\
\hline $\mathrm{H}$ & 4.70496 & -0.46561 & 3.58868 \\
\hline $\mathrm{C}$ & -4.56907 & 2.80084 & 1.06387 \\
\hline $\mathrm{H}$ & -5.48026 & 2.96299 & 0.47957 \\
\hline $\mathrm{H}$ & -4.64173 & 3.38694 & 1.98376 \\
\hline $\mathrm{C}$ & 1.54338 & -1.48560 & 2.85556 \\
\hline
\end{tabular}




$\begin{array}{lrrr}\mathrm{H} & 0.58959 & -1.69439 & 3.33143 \\ \mathrm{C} & -3.75122 & -3.88689 & -1.84876 \\ \mathrm{H} & -2.95662 & -4.54610 & -2.18701 \\ \mathrm{C} & -5.04652 & -4.04002 & -2.34134 \\ \mathrm{H} & -5.26750 & -4.83555 & -3.04700 \\ \mathrm{C} & -5.75631 & -2.14540 & -1.02615 \\ \mathrm{H} & -6.54262 & -1.47921 & -0.68260 \\ \mathrm{C} & -6.04879 & -3.15801 & -1.93888 \\ \mathrm{H} & -7.05883 & -3.26386 & -2.32389 \\ \mathrm{C} & 2.64629 & -1.10632 & 3.61879 \\ \mathrm{H} & 2.55681 & -1.02907 & 4.69878\end{array}$

P-2a, $E_{\text {el }}(B 3 L Y P-D 3 B J / 6-31 G(d)$, superfine integration grid $)=-2639.73927763$ Hartrees

\begin{tabular}{|c|c|c|c|}
\hline S & -3.51295 & 0.46481 & -2.25652 \\
\hline S & 3.30666 & 3.65306 & 0.03022 \\
\hline 0 & -3.63468 & 1.58836 & -3.20705 \\
\hline 0 & -4.18407 & -0.81690 & -2.52978 \\
\hline 0 & 3.16209 & 2.88409 & -1.21521 \\
\hline 0 & 3.69672 & 5.07359 & -0.00291 \\
\hline C & -0.92782 & 0.98131 & -1.20431 \\
\hline $\mathrm{C}$ & -0.07263 & 0.44096 & -0.22638 \\
\hline C & -0.44276 & -1.95994 & 0.66932 \\
\hline $\mathrm{C}$ & 0.25724 & -1.01462 & -0.10537 \\
\hline C & -2.87554 & -1.31179 & 0.85538 \\
\hline $\mathrm{H}$ & -2.99701 & -1.45929 & -0.21114 \\
\hline C & 1.47720 & -1.37868 & -0.68072 \\
\hline $\mathrm{H}$ & 2.03278 & -0.62539 & -1.22927 \\
\hline C & 0.71149 & 2.68438 & 0.32584 \\
\hline $\mathrm{C}$ & -5.25660 & -0.52745 & 0.93842 \\
\hline C & -5.30211 & 0.41040 & -0.11469 \\
\hline $\mathrm{C}$ & 0.72452 & 1.30627 & 0.53043 \\
\hline $\mathrm{H}$ & 1.41708 & 0.87552 & 1.24575 \\
\hline $\mathrm{C}$ & 4.29366 & 1.35011 & 1.32133 \\
\hline C & -3.98176 & -0.89032 & 1.60565 \\
\hline $\mathrm{C}$ & 0.08739 & -3.25369 & 0.78817 \\
\hline $\mathrm{H}$ & -0.45794 & -3.98540 & 1.37724 \\
\hline C & -0.16040 & 3.21515 & -0.63101 \\
\hline $\mathrm{H}$ & -0.17592 & 4.28557 & -0.81653 \\
\hline C & -0.97332 & 2.37253 & -1.37980 \\
\hline $\mathrm{H}$ & -1.62916 & 2.78584 & -2.14009 \\
\hline C & 1.71866 & 3.55052 & 1.01107 \\
\hline $\mathrm{H}$ & 1.99299 & 3.17978 & 2.00221 \\
\hline $\mathrm{H}$ & 1.41095 & 4.59550 & 1.08794 \\
\hline C & 3.69846 & -1.34166 & 1.79037 \\
\hline $\mathrm{H}$ & 3.46408 & -2.38544 & 1.96952 \\
\hline C & -1.64664 & -1.58352 & 1.46071 \\
\hline $\mathrm{C}$ & 2.04502 & -2.64026 & -0.49186 \\
\hline $\mathrm{C}$ & -4.07716 & 1.12083 & -0.60794 \\
\hline $\mathrm{H}$ & -3.23342 & 1.05150 & 0.07797 \\
\hline $\mathrm{H}$ & -4.27484 & 2.17264 & -0.82246 \\
\hline $\mathrm{C}$ & 1.31122 & -3.59763 & 0.21690 \\
\hline $\mathrm{H}$ & 1.72577 & -4.58988 & 0.37254 \\
\hline $\mathrm{C}$ & 3.78675 & 0.94456 & 2.56032 \\
\hline $\mathrm{H}$ & 3.63182 & 1.67937 & 3.34684 \\
\hline $\mathrm{C}$ & 4.21605 & -0.95950 & 0.54144 \\
\hline $\mathrm{C}$ & 3.48884 & -0.39921 & 2.79141 \\
\hline
\end{tabular}




$\begin{array}{lrrr}\text { H } & 3.10024 & -0.71076 & 3.75662 \\ \mathrm{C} & -6.45595 & -1.10037 & 1.38621 \\ \mathrm{H} & -6.41781 & -1.82280 & 2.19631 \\ \mathrm{C} & -6.53408 & 0.72314 & -0.70177 \\ \mathrm{H} & -6.55950 & 1.44360 & -1.51512 \\ \mathrm{C} & 4.50967 & 0.39055 & 0.32167 \\ \mathrm{H} & 4.87469 & 0.70747 & -0.65026 \\ \mathrm{C} & 4.46495 & -1.99024 & -0.50808 \\ \mathrm{C} & 3.44032 & -2.86290 & -0.94821 \\ \mathrm{C} & -7.71349 & 0.13133 & -0.26139 \\ \mathrm{H} & -8.65725 & 0.38391 & -0.73529 \\ \mathrm{C} & -7.67396 & -0.78228 & 0.79272 \\ \mathrm{H} & -8.58787 & -1.24955 & 1.14801 \\ \mathrm{C} & -1.71868 & 0.07943 & -2.10748 \\ \mathrm{H} & -1.64618 & -0.96532 & -1.80972 \\ \mathrm{H} & -1.39563 & 0.16274 & -3.15125 \\ \mathrm{C} & -3.85643 & -0.80005 & 2.99890 \\ \mathrm{H} & -4.70502 & -0.46584 & 3.58857 \\ \mathrm{C} & 4.56905 & 2.80077 & 1.06414 \\ \mathrm{H} & 5.48025 & 2.96296 & 0.47986 \\ \mathrm{H} & 4.64170 & 3.38681 & 1.98406 \\ \mathrm{C} & -1.54343 & -1.48579 & 2.85543 \\ \mathrm{H} & -0.58964 & -1.69460 & 3.33131 \\ \mathrm{C} & 3.75125 & -3.88677 & -1.84895 \\ \mathrm{H} & 2.95666 & -4.54596 & -2.18725 \\ \mathrm{C} & 5.04656 & -4.03986 & -2.34151 \\ \mathrm{H} & 5.26756 & -4.83535 & -3.04722 \\ \mathrm{C} & 5.75633 & -2.14534 & -1.02619 \\ \mathrm{H} & 6.54263 & -1.47916 & -0.68258 \\ \mathrm{C} & 6.04883 & -3.15788 & -1.93897 \\ \mathrm{H} & 7.05887 & -3.26370 & -2.32398 \\ \mathrm{C} & -2.64635 & -1.10656 & 3.61867 \\ \mathrm{H} & -2.55690 & -1.02937 & 4.69867 \\ & & & \end{array}$

\section{Potential Energy Minima of 1a,b, 2a,b, and 5-7.}

1a, $E_{\text {el }}(B 3 L Y P / 6-31 G(d)$, ultrafine integration grid $)=-2639.51758321$ Hartrees

$\begin{array}{rrrr}\mathrm{S} & -4.371565 & 0.554723 & -2.134292 \\ \mathrm{~S} & 2.733357 & 3.178155 & 0.255305 \\ \mathrm{O} & -5.588208 & 0.841229 & -2.915485 \\ \mathrm{O} & -3.716166 & -0.762109 & -2.254040 \\ \mathrm{O} & 2.961925 & 2.665130 & -1.108251 \\ \mathrm{O} & 2.394552 & 4.598972 & 0.457524 \\ \mathrm{C} & -1.141076 & 0.796060 & -1.437835 \\ \mathrm{C} & -0.079777 & 0.821898 & -0.527654 \\ \mathrm{C} & 0.088084 & -1.415841 & 0.642443 \\ \mathrm{C} & 0.636830 & -0.466122 & -0.250671 \\ \mathrm{C} & -2.437629 & -1.206290 & 0.931475 \\ \mathrm{H} & -2.583231 & -1.425224 & -0.121928 \\ \mathrm{C} & 1.853460 & -0.741033 & -0.891141 \\ \mathrm{H} & 2.269814 & 0.009551 & -1.556478 \\ \mathrm{C} & -0.559381 & 3.164655 & -0.122967 \\ \mathrm{C} & -4.962386 & -1.128567 & 1.224934 \\ \mathrm{C} & -5.517259 & -0.262257 & 0.256618 \\ \mathrm{C} & 0.231834 & 2.033863 & 0.123586\end{array}$




\begin{tabular}{|c|c|c|c|}
\hline C & 4.616865 & 1.397288 & 1.399191 \\
\hline C & -3.564904 & -1.014095 & 1.748031 \\
\hline C & 0.774111 & -2.623725 & 0.833218 \\
\hline $\mathrm{H}$ & 0.355290 & -3.361796 & 1.511926 \\
\hline C & -1.644492 & 3.107220 & -0.991842 \\
\hline $\mathrm{H}$ & -2.252882 & 3.993914 & -1.152445 \\
\hline C & -1.942695 & 1.919172 & -1.669569 \\
\hline C & 1.414461 & 2.153055 & 1.048489 \\
\hline C & 5.192301 & -1.327705 & 1.713620 \\
\hline $\mathrm{H}$ & 5.397773 & -2.387557 & 1.836105 \\
\hline C & -1.139692 & -1.159238 & 1.465076 \\
\hline C & 2.544655 & -1.941046 & -0.676704 \\
\hline C & -4.727664 & 0.867898 & -0.352690 \\
\hline $\mathrm{H}$ & -3.763503 & 1.012920 & 0.136229 \\
\hline $\mathrm{H}$ & -5.284741 & 1.810031 & -0.343389 \\
\hline C & 1.975261 & -2.889843 & 0.180629 \\
\hline $\mathrm{H}$ & 2.487750 & -3.832451 & 0.353544 \\
\hline C & 4.760872 & 0.847349 & 2.679654 \\
\hline H & 4.638378 & 1.479121 & 3.556441 \\
\hline C & 5.030936 & -0.800094 & 0.424964 \\
\hline C & 5.068966 & -0.504534 & 2.833014 \\
\hline $\mathrm{H}$ & 5.193547 & -0.921047 & 3.828894 \\
\hline C & -5.785359 & -2.124280 & 1.778073 \\
\hline $\mathrm{H}$ & -5.362334 & -2.790414 & 2.524758 \\
\hline C & -6.854992 & -0.427692 & -0.133590 \\
\hline $\mathrm{H}$ & -7.268835 & 0.239678 & -0.883813 \\
\hline C & 4.779852 & 0.568310 & 0.280728 \\
\hline $\mathrm{H}$ & 4.661834 & 0.985778 & -0.713242 \\
\hline C & 5.066702 & -1.683632 & -0.783192 \\
\hline C & 3.870405 & -2.213412 & -1.319054 \\
\hline C & -7.647939 & -1.431664 & 0.414224 \\
\hline $\mathrm{H}$ & -8.679312 & -1.544008 & 0.092168 \\
\hline C & -7.109423 & -2.284254 & 1.377978 \\
\hline $\mathrm{H}$ & -7.716372 & -3.071184 & 1.817538 \\
\hline C & -3.130590 & 1.841549 & -2.590759 \\
\hline C & -3.375085 & -0.771776 & 3.118417 \\
\hline $\mathrm{H}$ & -4.241408 & -0.617790 & 3.756025 \\
\hline C & 4.259120 & 2.851923 & 1.238380 \\
\hline $\mathrm{H}$ & 5.006664 & 3.418132 & 0.670038 \\
\hline $\mathrm{H}$ & 4.111838 & 3.352113 & 2.199331 \\
\hline $\mathrm{C}$ & -0.981710 & -0.920701 & 2.838653 \\
\hline $\mathrm{H}$ & 0.019310 & -0.891294 & 3.261126 \\
\hline C & 3.940534 & -3.046142 & -2.444188 \\
\hline $\mathrm{H}$ & 3.020085 & -3.454713 & -2.852645 \\
\hline C & 5.163467 & -3.348949 & -3.042749 \\
\hline $\mathrm{H}$ & 5.193122 & -3.992097 & -3.918151 \\
\hline C & 6.288867 & -2.000082 & -1.389895 \\
\hline $\mathrm{H}$ & 7.204991 & -1.590465 & -0.972500 \\
\hline C & 6.342213 & -2.824616 & -2.513676 \\
\hline $\mathrm{H}$ & 7.300192 & -3.054901 & -2.972014 \\
\hline C & -2.092076 & -0.722752 & 3.65815 \\
\hline $\mathrm{H}$ & -1.956644 & -0.532854 & 4.719790 \\
\hline $\mathrm{H}$ & 1.172982 & 2.675345 & 1.978908 \\
\hline $\mathrm{H}$ & 1.862022 & 1.184000 & 1.275029 \\
\hline $\mathrm{H}$ & -3.678695 & 2.785928 & -2.643427 \\
\hline $\mathrm{H}$ & -2.863474 & 1.541870 & -3.610873 \\
\hline $\mathrm{H}$ & -1.370884 & -0.132750 & -1.94813 \\
\hline $\mathrm{H}$ & -0.312785 & 4.100958 & 0.36873 \\
\hline
\end{tabular}


1b, $E_{\text {el }}(B 3 L Y P / 6-31 G(d)$, ultrafine integration grid $)=-2338.73757691$ Hartrees

\begin{tabular}{rrr}
-4.402448 & 0.300879 & -2.517129 \\
2.527084 & 3.381463 & -0.166900 \\
-1.301990 & 0.829006 & -1.704009 \\
-0.258170 & 0.969663 & -0.786480 \\
-0.011154 & -1.092298 & 0.668281 \\
0.489299 & -0.255989 & -0.356131 \\
-2.548135 & -0.964695 & 0.824810 \\
-2.623849 & -1.268104 & -0.214682 \\
1.717470 & -0.564632 & -0.957355 \\
2.089139 & 0.088016 & -1.742013 \\
-0.779784 & 3.325953 & -0.641905 \\
-5.083319 & -1.015404 & 0.982915 \\
-5.618052 & -0.293076 & -0.107897 \\
0.023755 & 2.246912 & -0.252748 \\
4.432798 & 1.809001 & 1.279568 \\
-3.725893 & -0.772244 & 1.564614 \\
0.752431 & -2.205168 & 1.051616 \\
0.373067 & -2.854908 & 1.835817 \\
-1.843490 & 3.159488 & -1.527109 \\
-2.466309 & 4.012214 & -1.788037 \\
-2.116833 & 1.902905 & -2.075462 \\
1.162361 & 2.483067 & 0.710624 \\
5.508434 & -0.748139 & 1.728157 \\
5.912578 & -1.741205 & 1.905767 \\
-1.281918 & -0.817382 & 1.413300 \\
2.484809 & -1.664583 & -0.556220 \\
-4.865315 & 0.819166 & -0.799664 \\
-3.953218 & 1.079120 & -0.260803 \\
-5.492486 & 1.714963 & -0.871547 \\
1.977228 & -2.489933 & 0.454465 \\
2.550842 & -3.353385 & 0.780339 \\
4.916385 & 1.452322 & 2.545182 \\
4.871922 & 2.170242 & 3.361578 \\
5.004911 & -0.421851 & 0.461024 \\
5.462291 & 0.186758 & 2.762728 \\
5.839687 & -0.076926 & 3.747407 \\
-5.882711 & -1.996941 & 1.595570 \\
-5.470380 & -2.551851 & 2.433836 \\
-6.917737 & -0.588340 & -0.547332 \\
-7.325204 & -0.024183 & -1.382896 \\
4.490190 & 0.863586 & 0.251386 \\
4.115584 & 1.132753 & -0.730302 \\
5.015207 & -1.419391 & -0.652490 \\
3.818785 & -1.972847 & -1.165596 \\
-7.689937 & -1.573775 & 0.061154 \\
-8.692341 & -1.781716 & -0.303182 \\
-7.167826 & -2.283031 & 1.142981 \\
-7.756298 & -3.054551 & 1.632326 \\
-3.292412 & 1.695973 & -3.003234 \\
-3.618063 & -0.416492 & 2.919052 \\
-4.522532 & -0.257724 & 3.500010 \\
3.903245 & 3.212729 & 1.042938 \\
\hline & 3.851654 & 0.619415 \\
\hline & -0.670331 & 1.994127 \\
\hline
\end{tabular}




$\begin{array}{rrrr}\mathrm{H} & -0.232686 & -0.357037 & 3.237558 \\ \mathrm{C} & 3.892317 & -2.895553 & -2.218432 \\ \mathrm{H} & 2.970613 & -3.323305 & -2.604055 \\ \mathrm{C} & 5.117850 & -3.276320 & -2.764351 \\ \mathrm{H} & 5.148458 & -3.990903 & -3.582552 \\ \mathrm{C} & 6.240897 & -1.824134 & -1.201399 \\ \mathrm{H} & 7.157684 & -1.397321 & -0.803044 \\ \mathrm{C} & 6.298313 & -2.742212 & -2.249058 \\ \mathrm{H} & 7.260208 & -3.034149 & -2.662139 \\ \mathrm{C} & -2.368248 & -0.262385 & 3.514647 \\ \mathrm{H} & -2.297908 & 0.016685 & 4.562835 \\ \mathrm{H} & 0.836000 & 3.094601 & 1.558663 \\ \mathrm{H} & 1.564121 & 1.541913 & 1.090405 \\ \mathrm{H} & -3.878157 & 2.617067 & -3.092064 \\ \mathrm{H} & -2.963484 & 1.422106 & -4.012888 \\ \mathrm{H} & -1.514972 & -0.156660 & -2.106825 \\ \mathrm{H} & -0.575026 & 4.311361 & -0.230189\end{array}$

2a, $E_{\text {el }}(B 3 L Y P / 6-31 G(d)$, ultrafine integration grid $)=-2639.50529214$ Hartrees

$\begin{array}{rrrr}\mathrm{S} & -3.561381 & 0.760454 & -2.207346 \\ \mathrm{~S} & 3.507652 & 3.590573 & 0.078808 \\ \mathrm{O} & -3.592898 & 2.002103 & -3.007273 \\ \mathrm{O} & -4.232651 & -0.454276 & -2.699393 \\ \mathrm{O} & 3.301573 & 2.887696 & -1.197638 \\ \mathrm{O} & 3.945083 & 4.998161 & 0.097728 \\ \mathrm{C} & -0.939090 & 1.129991 & -1.075407 \\ \mathrm{C} & -0.075954 & 0.519078 & -0.140307 \\ \mathrm{C} & -0.527374 & -1.910878 & 0.694748 \\ \mathrm{C} & 0.202449 & -0.956970 & -0.048363 \\ \mathrm{C} & -2.973006 & -1.288333 & 0.864351 \\ \mathrm{H} & -3.070501 & -1.400247 & -0.209920 \\ \mathrm{C} & 1.417208 & -1.342183 & -0.631157 \\ \mathrm{H} & 1.986503 & -0.591814 & -1.170250 \\ \mathrm{C} & 0.856765 & 2.713183 & 0.427070 \\ \mathrm{C} & -5.377562 & -0.571552 & 0.910161 \\ \mathrm{C} & -5.436515 & 0.405560 & -0.110304 \\ \mathrm{C} & 0.792655 & 1.331185 & 0.602726 \\ \mathrm{H} & 1.483265 & 0.849328 & 1.288505 \\ \mathrm{C} & 4.524036 & 1.202243 & 1.275802 \\ \mathrm{C} & -4.106080 & -0.915770 & 1.602094 \\ \mathrm{C} & -0.023215 & -3.220071 & 0.774853 \\ \mathrm{H} & -0.587921 & -3.959266 & 1.336655 \\ \mathrm{C} & -0.027340 & 3.310750 & -0.478658 \\ \mathrm{H} & 0.002348 & 4.384966 & -0.642105 \\ \mathrm{C} & -0.913774 & 2.528531 & -1.210834 \\ \mathrm{H} & -1.571380 & 2.997847 & -1.936403 \\ \mathrm{C} & 1.933204 & 3.507896 & 1.101464 \\ \mathrm{H} & 2.220505 & 3.093354 & 2.072368 \\ \mathrm{H} & 1.677617 & 4.563122 & 1.224149 \\ \mathrm{C} & 3.981215 & -1.501419 & 1.766083 \\ \mathrm{H} & 3.772298 & -2.549508 & 1.957591 \\ \mathrm{C} & -1.748129 & -1.558934 & 1.482349 \\ \mathrm{C} & 1.953176 & -2.625484 & -0.490339 \\ \mathrm{C} & -4.242022 & 1.220479 & -0.525392\end{array}$




\begin{tabular}{|c|c|c|c|}
\hline $\mathrm{H}$ & -3.413689 & 1.164481 & 0.182237 \\
\hline $\mathrm{H}$ & -4.507295 & 2.270007 & -0.672857 \\
\hline $\mathrm{C}$ & 1.191750 & -3.580756 & 0.194450 \\
\hline $\mathrm{H}$ & 1.570106 & -4.592704 & 0.314788 \\
\hline $\mathrm{C}$ & 4.179275 & 0.770420 & 2.562998 \\
\hline $\mathrm{H}$ & 4.132857 & 1.487957 & 3.379487 \\
\hline $\mathrm{C}$ & 4.327938 & -1.095569 & 0.465981 \\
\hline C & 3.913397 & -0.578022 & 2.805763 \\
\hline $\mathrm{H}$ & 3.659292 & -0.908548 & 3.809217 \\
\hline C & -6.563091 & -1.216896 & 1.297368 \\
\hline $\mathrm{H}$ & -6.515672 & -1.969443 & 2.079681 \\
\hline C & -6.669540 & 0.683371 & -0.717701 \\
\hline $\mathrm{H}$ & -6.712506 & 1.438752 & -1.498391 \\
\hline $\mathrm{C}$ & 4.608158 & 0.258634 & 0.238760 \\
\hline $\mathrm{H}$ & 4.857596 & 0.592574 & -0.764262 \\
\hline C & 4.425921 & -2.112014 & -0.629084 \\
\hline C & 3.317117 & -2.903494 & -1.023199 \\
\hline C & -7.832004 & 0.019980 & -0.337036 \\
\hline $\mathrm{H}$ & -8.773296 & 0.249572 & -0.828148 \\
\hline C & -7.778671 & -0.933189 & 0.680863 \\
\hline $\mathrm{H}$ & -8.678846 & -1.456699 & 0.991213 \\
\hline $\mathrm{C}$ & -1.782223 & 0.298626 & -2.008011 \\
\hline $\mathrm{H}$ & -1.763301 & -0.758918 & -1.749019 \\
\hline $\mathrm{H}$ & -1.440562 & 0.394898 & -3.045908 \\
\hline $\mathrm{C}$ & -4.011339 & -0.872954 & 3.001095 \\
\hline $\mathrm{H}$ & -4.877948 & -0.579598 & 3.587183 \\
\hline C & 4.795150 & 2.659565 & 1.026602 \\
\hline $\mathrm{H}$ & 5.674761 & 2.822966 & 0.394550 \\
\hline $\mathrm{H}$ & 4.935666 & 3.221795 & 1.953983 \\
\hline $\mathrm{C}$ & -1.680036 & -1.510701 & 2.883251 \\
\hline $\mathrm{H}$ & -0.736643 & -1.723818 & 3.378362 \\
\hline $\mathrm{C}$ & 3.507330 & -3.918139 & -1.971229 \\
\hline $\mathrm{H}$ & 2.652435 & -4.515480 & -2.277285 \\
\hline $\mathrm{C}$ & 4.755321 & -4.141607 & -2.551407 \\
\hline $\mathrm{H}$ & 4.876177 & -4.928756 & -3.290592 \\
\hline $\mathrm{C}$ & 5.669200 & -2.338102 & -1.236165 \\
\hline $\mathrm{H}$ & 6.519491 & -1.734774 & -0.929327 \\
\hline C & 5.837785 & -3.339437 & -2.192097 \\
\hline $\mathrm{H}$ & 6.813105 & -3.497785 & -2.643994 \\
\hline $\mathrm{C}$ & -2.807325 & -1.178617 & 3.634371 \\
\hline $\mathrm{H}$ & -2.742220 & -1.140544 & 4.718613 \\
\hline
\end{tabular}

2b, $E_{\text {el }}(B 3 L Y P / 6-31 G(d)$, ultrafine integration grid $)=-2338.72788922$ Hartrees

$\begin{array}{lrrr}\mathrm{S} & -3.569851 & 0.663846 & -2.626163 \\ \mathrm{~S} & 3.255372 & 4.026304 & -0.402357 \\ \mathrm{C} & -1.030544 & 1.200501 & -1.360774 \\ \mathrm{C} & -0.114602 & 0.724879 & -0.397815 \\ \mathrm{C} & -0.449438 & -1.634761 & 0.655264 \\ \mathrm{C} & 0.245794 & -0.717430 & -0.164736 \\ \mathrm{C} & -2.927659 & -1.135463 & 0.681959 \\ \mathrm{H} & -2.967135 & -1.318438 & -0.384837 \\ \mathrm{C} & 1.498905 & -1.087848 & -0.670209 \\ \mathrm{H} & 2.041388 & -0.365118 & -1.270764 \\ \mathrm{C} & 0.668192 & 3.015712 & 0.007334\end{array}$




\begin{tabular}{|c|c|c|c|}
\hline $\mathrm{C}$ & -5.375928 & -0.596720 & 0.574845 \\
\hline $\mathrm{C}$ & -5.466279 & 0.278018 & -0.533290 \\
\hline $\mathrm{C}$ & 0.704781 & 1.646895 & 0.271512 \\
\hline $\mathrm{H}$ & 1.432988 & 1.265600 & 0.981028 \\
\hline $\mathrm{C}$ & 4.364973 & 1.830877 & 1.082522 \\
\hline $\mathrm{C}$ & -4.113242 & -0.783380 & 1.341549 \\
\hline $\mathrm{C}$ & 0.129755 & -2.894033 & 0.886920 \\
\hline $\mathrm{H}$ & -0.408677 & -3.604581 & 1.508563 \\
\hline $\mathrm{C}$ & -0.280622 & 3.480985 & -0.910910 \\
\hline $\mathrm{H}$ & -0.347541 & 4.543730 & -1.133575 \\
\hline $\mathrm{C}$ & -1.115398 & 2.585359 & -1.572427 \\
\hline $\mathrm{H}$ & -1.832577 & 2.955292 & -2.300127 \\
\hline $\mathrm{C}$ & 1.693750 & 3.935328 & 0.619741 \\
\hline $\mathrm{H}$ & 1.979391 & 3.606217 & 1.622633 \\
\hline $\mathrm{H}$ & 1.315249 & 4.958516 & 0.695016 \\
\hline $\mathrm{C}$ & 3.988352 & -0.850411 & 1.822016 \\
\hline $\mathrm{H}$ & 3.841180 & -1.887535 & 2.107753 \\
\hline $\mathrm{C}$ & -1.717803 & -1.287572 & 1.366181 \\
\hline $\mathrm{C}$ & 2.107881 & -2.311492 & -0.378596 \\
\hline $\mathrm{C}$ & -4.323516 & 1.156966 & -0.992750 \\
\hline $\mathrm{H}$ & -3.519302 & 1.213791 & -0.259194 \\
\hline $\mathrm{H}$ & -4.694851 & 2.174324 & -1.147515 \\
\hline $\mathrm{C}$ & 1.383783 & -3.236622 & 0.383208 \\
\hline $\mathrm{H}$ & 1.818892 & -4.203609 & 0.623005 \\
\hline $\mathrm{C}$ & 4.016405 & 1.496700 & 2.396100 \\
\hline $\mathrm{H}$ & 3.897622 & 2.283772 & 3.137616 \\
\hline $\mathrm{C}$ & 4.347315 & -0.545210 & 0.496964 \\
\hline $\mathrm{C}$ & 3.830340 & 0.161521 & 2.763319 \\
\hline $\mathrm{H}$ & 3.565548 & -0.087277 & 3.787648 \\
\hline $\mathrm{C}$ & -6.521557 & -1.303673 & 0.978752 \\
\hline $\mathrm{H}$ & -6.444105 & -1.977634 & 1.827803 \\
\hline $\mathrm{C}$ & -6.696943 & 0.396727 & -1.196465 \\
\hline $\mathrm{H}$ & -6.772316 & 1.078266 & -2.041065 \\
\hline $\mathrm{C}$ & 4.540544 & 0.797389 & 0.147682 \\
\hline $\mathrm{H}$ & 4.799101 & 1.047843 & -0.878612 \\
\hline $\mathrm{C}$ & 4.547544 & -1.653376 & -0.490650 \\
\hline $\mathrm{C}$ & 3.505326 & -2.550454 & -0.839264 \\
\hline $\mathrm{C}$ & -7.818890 & -0.323992 & -0.797106 \\
\hline $\mathrm{H}$ & -8.756318 & -0.212460 & -1.335316 \\
\hline $\mathrm{C}$ & -7.731754 & -1.178567 & 0.302719 \\
\hline $\mathrm{H}$ & -8.598833 & -1.745684 & 0.630506 \\
\hline C & -1.804505 & 0.248267 & -2.247738 \\
\hline $\mathrm{H}$ & -1.726688 & -0.773098 & -1.875278 \\
\hline $\mathrm{H}$ & -1.363579 & 0.242015 & -3.252963 \\
\hline $\mathrm{C}$ & -4.082151 & -0.626663 & 2.735359 \\
\hline $\mathrm{H}$ & -4.989732 & -0.345463 & 3.262646 \\
\hline $\mathrm{C}$ & 4.545776 & 3.281075 & 0.701894 \\
\hline $\mathrm{H}$ & 5.460237 & 3.420540 & 0.114513 \\
\hline $\mathrm{H}$ & 4.640710 & 3.901084 & 1.598773 \\
\hline $\mathrm{C}$ & -1.714299 & -1.128861 & 2.760663 \\
\hline $\mathrm{H}$ & -0.783227 & -1.246321 & 3.308369 \\
\hline $\mathrm{C}$ & 3.793558 & -3.637279 & -1.676195 \\
\hline $\mathrm{H}$ & 2.989345 & -4.316785 & -1.946474 \\
\hline $\mathrm{C}$ & 5.073473 & -3.833909 & -2.193274 \\
\hline $\mathrm{H}$ & 5.270315 & -4.679661 & -2.846614 \\
\hline $\mathrm{C}$ & 5.823894 & -1.856156 & -1.034489 \\
\hline $\mathrm{H}$ & 6.623126 & -1.172122 & -0.761347 \\
\hline $\mathrm{C}$ & 6.089936 & -2.931649 & -1.881730 \\
\hline
\end{tabular}




$\begin{array}{rrrr}\mathrm{H} & 7.089604 & -3.069175 & -2.284966 \\ \mathrm{C} & -2.890641 & -0.806518 & 3.437310 \\ \mathrm{H} & -2.875181 & -0.679403 & 4.516720\end{array}$

5a, $E_{\text {el }}(B 3 L Y P / 6-31 G(d)$, ultrafine integration grid $)=-1551.40233825$ Hartrees

\begin{tabular}{|c|c|c|c|}
\hline S & -3.432484 & 0.647008 & -2.303460 \\
\hline O & -3.535604 & 1.838311 & -3.172124 \\
\hline 0 & -4.063338 & -0.621163 & -2.707163 \\
\hline C & -0.868153 & 1.112470 & -1.116920 \\
\hline $\mathrm{C}$ & -0.075457 & 0.500931 & -0.120512 \\
\hline C & -0.436485 & -1.883270 & 0.766984 \\
\hline $\mathrm{C}$ & 0.224663 & -0.973074 & -0.087992 \\
\hline C & -2.838266 & -1.216074 & 0.935899 \\
\hline $\mathrm{H}$ & -2.915817 & -1.365399 & -0.136335 \\
\hline $\mathrm{C}$ & 1.292551 & -1.436788 & -0.873217 \\
\hline $\mathrm{H}$ & 1.806942 & -0.734022 & -1.523495 \\
\hline $\mathrm{C}$ & 0.449513 & 2.704716 & 0.785873 \\
\hline $\mathrm{C}$ & -5.242855 & -0.503925 & 0.922012 \\
\hline $\mathrm{C}$ & -5.293014 & 0.391967 & -0.171837 \\
\hline $\mathrm{C}$ & 0.568259 & 1.317318 & 0.822831 \\
\hline $\mathrm{H}$ & 1.181160 & 0.846798 & 1.586551 \\
\hline $\mathrm{C}$ & -3.977764 & -0.810985 & 1.643195 \\
\hline $\mathrm{C}$ & 0.009668 & -3.212201 & 0.826221 \\
\hline $\mathrm{H}$ & -0.505741 & -3.905846 & 1.485064 \\
\hline C & -0.307631 & 3.306218 & -0.220198 \\
\hline $\mathrm{H}$ & -0.393248 & 4.388077 & -0.272573 \\
\hline C & -0.956419 & 2.513492 & -1.163164 \\
\hline $\mathrm{H}$ & -1.553713 & 2.976627 & -1.943822 \\
\hline $\mathrm{H}$ & 0.958361 & 3.312245 & 1.529325 \\
\hline $\mathrm{C}$ & -1.623119 & -1.478504 & 1.578305 \\
\hline C & 1.714665 & -2.764037 & -0.816459 \\
\hline $\mathrm{C}$ & -4.096972 & 1.179376 & -0.633515 \\
\hline $\mathrm{H}$ & -3.265688 & 1.149922 & 0.070863 \\
\hline $\mathrm{H}$ & -4.357816 & 2.223393 & -0.821896 \\
\hline $\mathrm{C}$ & 1.075451 & -3.654876 & 0.045232 \\
\hline $\mathrm{H}$ & 1.398083 & -4.690785 & 0.104207 \\
\hline $\mathrm{C}$ & -6.434083 & -1.113044 & 1.349836 \\
\hline $\mathrm{H}$ & -6.393978 & -1.804963 & 2.186538 \\
\hline $\mathrm{C}$ & -6.521986 & 0.626007 & -0.805609 \\
\hline $\mathrm{H}$ & -6.557821 & 1.320271 & -1.641519 \\
\hline $\mathrm{H}$ & 2.544062 & -3.095603 & -1.435205 \\
\hline $\mathrm{C}$ & -7.689266 & -0.001815 & -0.382123 \\
\hline $\mathrm{H}$ & -8.626842 & 0.192529 & -0.895122 \\
\hline $\mathrm{C}$ & -7.645274 & -0.873153 & 0.707025 \\
\hline $\mathrm{H}$ & -8.549009 & -1.368184 & 1.051918 \\
\hline $\mathrm{C}$ & -1.621274 & 0.280372 & -2.122281 \\
\hline $\mathrm{H}$ & -1.550544 & -0.786491 & -1.911622 \\
\hline $\mathrm{H}$ & -1.279944 & 0.451871 & -3.149886 \\
\hline $\mathrm{C}$ & -3.894184 & -0.715804 & 3.041496 \\
\hline $\mathrm{H}$ & -4.763099 & -0.394313 & 3.609193 \\
\hline $\mathrm{C}$ & -1.565702 & -1.382397 & 2.974931 \\
\hline $\mathrm{H}$ & -0.631434 & -1.589746 & 3.489767 \\
\hline $\mathrm{C}$ & -2.699412 & -1.008142 & 3.698445 \\
\hline $\mathrm{H}$ & -2.646395 & -0.928386 & 4.781067 \\
\hline
\end{tabular}


5b, $E_{\text {el }}(B 3 L Y P / 6-31 G(d)$, ultrafine integration grid $)=-1401.01304386$ Hartrees

\begin{tabular}{|c|c|c|}
\hline-0.813605 & -1.820563 & 1.708613 \\
\hline 1.371204 & -2.078858 & -0.169330 \\
\hline 2.056637 & -0.903220 & -0.551592 \\
\hline 2.075896 & 1.460656 & 0.419456 \\
\hline 2.570766 & 0.134135 & 0.405936 \\
\hline-0.366692 & 1.210184 & 0.110715 \\
\hline-0.336561 & 0.553764 & 0.972274 \\
\hline 3.682535 & -0.180929 & 1.202216 \\
\hline 4.075294 & -1.194333 & 1.1765 \\
\hline 2.040308 & -1.664861 & -2.875885 \\
\hline-2.825854 & 0.749345 & -0.0620 \\
\hline-2.871733 & -0.632794 & 0.25944 \\
\hline 2.371828 & -0.719681 & -1.90925 \\
\hline 2.903247 & 0.182096 & -2.199375 \\
\hline-1.588919 & 1.435191 & -0.532398 \\
\hline 2.732645 & 2.426030 & 1.195 \\
\hline 2.343261 & 3.440702 & 1.20517 \\
\hline 1.385939 & -2.836521 & -2.4949 \\
\hline 1.131930 & -3.593055 & -3.23238 \\
\hline 1.059971 & -3.029963 & -1.1562 \\
\hline 0.542985 & -3.938970 & -0.85642 \\
\hline 2.3036 & -1.492643 & -3.915 \\
\hline 0.821232 & 1.818641 & -0.306028 \\
\hline 4.310861 & 0.785046 & 1.987701 \\
\hline-1.694417 & -1.568781 & 0.0874 \\
\hline-0.976222 & -1.173645 & -0.6308 \\
\hline-2.044512 & -2.539190 & -0.27507 \\
\hline 3.842332 & 2.098334 & \\
\hline 4.329653 & 2.862403 & 2.57304 \\
\hline-4.002968 & 1.506209 & 0.0676 \\
\hline-3.966717 & 2.566600 & -0.16630 \\
\hline-4.081284 & -1.182095 & 0.7 \\
\hline-4.113240 & -2.242812 & 0.94629 \\
\hline 5.171787 & 0.514669 & 2.59322 \\
\hline-5.234313 & -0.412434 & 0.84383 \\
\hline-6.15 & -0.868729 & 1.197 \\
\hline-5.194463 & 0.942233 & 0.51647 \\
\hline-6.083282 & 1.559784 & 0.61473 \\
\hline 0.927138 & -2.367524 & 1.241985 \\
\hline 1.558856 & -1.860949 & 1.974354 \\
\hline 0.979514 & -3.443140 & 1.42886 \\
\hline-1.609393 & 2.343404 & -1.60527 \\
\hline-2.538545 & 2.527645 & -2.13797 \\
\hline 0.774562 & 2.734586 & -1.36403 \\
\hline 1.684869 & 3.225541 & -1.69890 \\
\hline-0.440595 & 2.992264 & -2.00437 \\
\hline-0.472575 & 3.691685 & -2.83589 \\
\hline
\end{tabular}

6a, $E_{\mathrm{el}}(\mathrm{B} 3 \mathrm{LYP} / 6-31 \mathrm{G}(\mathrm{d})$, ultrafine integration grid $)=-1551.41065348$ Hartrees

$\begin{array}{rrrr}\mathrm{S} & -4.363773 & 0.598453 & -2.165009 \\ \mathrm{O} & -5.594072 & 0.908551 & -2.916247 \\ \mathrm{O} & -3.722624 & -0.721460 & -2.319084 \\ \mathrm{C} & -1.139416 & 0.794791 & -1.516069\end{array}$




$\begin{array}{lrrr}\mathrm{C} & -0.127906 & 0.774255 & -0.552118 \\ \mathrm{C} & 0.135420 & -1.410243 & 0.699443 \\ \mathrm{C} & 0.605797 & -0.499121 & -0.273539 \\ \mathrm{C} & -2.383741 & -1.182267 & 0.959749 \\ \mathrm{H} & -2.508883 & -1.383702 & -0.099987 \\ \mathrm{C} & 1.782797 & -0.789582 & -0.975965 \\ \mathrm{H} & 2.136872 & -0.081284 & -1.720412 \\ \mathrm{C} & -0.598234 & 3.102293 & -0.057539 \\ \mathrm{C} & -4.916158 & -1.107235 & 1.216909 \\ \mathrm{C} & -5.466065 & -0.246910 & 0.239847 \\ \mathrm{C} & 0.147536 & 1.945303 & 0.167804 \\ \mathrm{C} & -3.524451 & -0.996884 & 1.758202 \\ \mathrm{C} & 0.875829 & -2.578148 & 0.940712 \\ \mathrm{H} & 0.515070 & -3.279206 & 1.688575 \\ \mathrm{C} & -1.648247 & 3.092959 & -0.976427 \\ \mathrm{H} & -2.252455 & 3.985758 & -1.120581 \\ \mathrm{C} & -1.930370 & 1.934555 & -1.711474 \\ \mathrm{H} & 0.937948 & 1.941937 & 0.913175 \\ \mathrm{C} & -1.094176 & -1.159878 & 1.518128 \\ \mathrm{C} & 2.499886 & -1.960105 & -0.731493 \\ \mathrm{C} & -4.678375 & 0.885185 & -0.369751 \\ \mathrm{H} & -3.703827 & 1.014570 & 0.102195 \\ \mathrm{H} & -5.226433 & 1.832157 & -0.333596 \\ \mathrm{C} & 2.043942 & -2.857653 & 0.233209 \\ \mathrm{H} & 2.592302 & -3.774150 & 0.433911 \\ \mathrm{C} & -5.743671 & -2.100918 & 1.768726 \\ \mathrm{H} & -5.324938 & -2.763926 & 2.520533 \\ \mathrm{C} & -6.802316 & -0.415029 & -0.155640 \\ \mathrm{H} & -7.212257 & 0.248826 & -0.911004 \\ \mathrm{H} & 3.408669 & -2.166837 & -1.290243 \\ \mathrm{C} & -7.598614 & -1.416037 & 0.392151 \\ \mathrm{H} & -8.628181 & -1.529572 & 0.064677 \\ \mathrm{C} & -7.065217 & -2.263953 & 1.362646 \\ \mathrm{H} & -7.673767 & -3.049395 & 1.802712 \\ \mathrm{C} & -3.119102 & 1.883028 & -2.631829 \\ \mathrm{C} & -3.355340 & -0.780622 & 3.136388 \\ \mathrm{H} & -4.231251 & -0.630067 & 3.761532 \\ \mathrm{C} & -0.958424 & -0.950704 & 2.898213 \\ \mathrm{H} & 0.035183 & -0.934309 & 3.338062 \\ \mathrm{C} & -2.082137 & -0.755535 & 3.700113 \\ \mathrm{H} & -1.964468 & -0.584450 & 4.767017 \\ \mathrm{H} & -3.661376 & 2.831331 & -2.671922 \\ \mathrm{H} & -2.865901 & 1.584747 & -3.655750 \\ \mathrm{H} & -1.357679 & -0.107221 & -2.077540 \\ \mathrm{H} & -0.377307 & 4.007414 & 0.501645 \\ & & & \end{array}$

6b, $E_{\text {el }}(B 3 L Y P / 6-31 G(d)$, ultrafine integration grid $)=-1401.02017083$ Hartrees

$\begin{array}{rrrr}\text { S } & -1.950896 & 1.994095 & -0.775311 \\ \mathrm{C} & 1.211339 & 1.811502 & -0.127859 \\ \mathrm{C} & 2.349167 & 1.216928 & 0.422686 \\ \mathrm{C} & 2.728874 & -1.137548 & -0.458424 \\ \mathrm{C} & 3.129811 & 0.215602 & -0.368096 \\ \mathrm{C} & 0.215964 & -1.371900 & -0.113340 \\ \mathrm{H} & 0.040316 & -0.686345 & -0.936201 \\ \mathrm{C} & 4.303573 & 0.625897 & -1.016361\end{array}$




$\begin{array}{rrrr}\mathrm{H} & 4.606636 & 1.666506 & -0.935426 \\ \mathrm{C} & 1.966162 & 2.469864 & 2.462382 \\ \mathrm{C} & -2.295792 & -1.694461 & 0.100816 \\ \mathrm{C} & -2.914443 & -0.424358 & 0.142645 \\ \mathrm{C} & 2.731341 & 1.566290 & 1.726659 \\ \mathrm{C} & -0.886453 & -1.955040 & 0.531727 \\ \mathrm{C} & 3.525512 & -2.027147 & -1.197704 \\ \mathrm{H} & 3.218366 & -3.067413 & -1.265159 \\ \mathrm{C} & 0.804587 & 3.018957 & 1.914388 \\ \mathrm{H} & 0.195009 & 3.699046 & 2.505431 \\ \mathrm{C} & 0.415079 & 2.694624 & 0.610351 \\ \mathrm{H} & 3.616052 & 1.113008 & 2.165389 \\ \mathrm{C} & 1.532687 & -1.690815 & 0.255228 \\ \mathrm{C} & 5.077185 & -0.268706 & -1.754112 \\ \mathrm{C} & -2.205164 & 0.816813 & 0.632759 \\ \mathrm{H} & -1.231344 & 0.578611 & 1.062307 \\ \mathrm{H} & -2.807309 & 1.322487 & 1.396132 \\ \mathrm{C} & 4.684224 & -1.603310 & -1.845825 \\ \mathrm{H} & 5.275754 & -2.313309 & -2.417767 \\ \mathrm{C} & -3.055865 & -2.798252 & -0.325686 \\ \mathrm{H} & -2.578895 & -3.774005 & -0.358298 \\ \mathrm{C} & -4.256879 & -0.308907 & -0.250165 \\ \mathrm{H} & -4.728766 & 0.669887 & -0.208581 \\ \mathrm{H} & 5.979853 & 0.075521 & -2.251834 \\ \mathrm{C} & -4.990492 & -1.409679 & -0.683184 \\ \mathrm{H} & -6.027625 & -1.288127 & -0.983818 \\ \mathrm{C} & -4.384150 & -2.665465 & -0.720672 \\ \mathrm{H} & -4.940757 & -3.537122 & -1.054459 \\ \mathrm{C} & -0.861679 & 3.255189 & 0.022015 \\ \mathrm{C} & -0.650669 & -2.872236 & 1.568729 \\ \mathrm{H} & -1.496050 & -3.324908 & 2.079820 \\ \mathrm{C} & 1.734951 & -2.615386 & 1.291060 \\ \mathrm{H} & 2.749757 & -2.870568 & 1.584053 \\ \mathrm{C} & 0.650445 & -3.196051 & 1.946703 \\ \mathrm{H} & 0.820522 & -3.903689 & 2.754047 \\ \mathrm{H} & -1.427455 & 3.799653 & 0.785442 \\ \mathrm{H} & -0.652279 & 3.961576 & -0.790498 \\ \mathrm{H} & 0.913481 & 1.552437 & -1.139335 \\ \mathrm{H} & 2.261642 & 2.731078 & 3.475194 \\ & & & \end{array}$

\section{7a, $E_{\mathrm{el}}(\mathrm{B} 3 \mathrm{LYP} / 6-31 \mathrm{G}(\mathrm{d})$, ultrafine integration grid $)=-1551.40993195$ Hartrees}

$\begin{array}{rrrr}\mathrm{S} & 3.542361 & 3.543255 & -0.159897 \\ \mathrm{O} & 3.277988 & 2.726029 & -1.354725 \\ \mathrm{O} & 4.007170 & 4.936297 & -0.290734 \\ \mathrm{C} & -1.208624 & 1.145544 & -0.542545 \\ \mathrm{C} & -0.020108 & 0.547394 & -0.097764 \\ \mathrm{C} & -0.651511 & -1.911901 & 0.148823 \\ \mathrm{C} & 0.267146 & -0.906814 & -0.185076 \\ \mathrm{C} & 1.558857 & -1.285666 & -0.574056 \\ \mathrm{H} & 2.244414 & -0.514809 & -0.903728 \\ \mathrm{C} & 0.872227 & 2.764413 & 0.426857 \\ \mathrm{C} & 0.986581 & 1.374758 & 0.414900 \\ \mathrm{H} & 1.888522 & 0.916924 & 0.803233 \\ \mathrm{C} & 4.592540 & 1.245230 & 1.185370 \\ \mathrm{C} & -0.256219 & -3.250939 & 0.122638\end{array}$




$\begin{array}{rrrr}\mathrm{H} & -0.967433 & -4.026127 & 0.395942 \\ \mathrm{C} & -0.309939 & 3.345772 & -0.046184 \\ \mathrm{H} & -0.421183 & 4.427226 & -0.052863 \\ \mathrm{C} & -1.349297 & 2.533570 & -0.507373 \\ \mathrm{H} & -2.267097 & 2.990398 & -0.867502 \\ \mathrm{C} & 2.018386 & 3.595084 & 0.930021 \\ \mathrm{H} & 2.360093 & 3.276872 & 1.920862 \\ \mathrm{H} & 1.783494 & 4.661388 & 0.966388 \\ \mathrm{C} & 3.993819 & -1.412627 & 1.848697 \\ \mathrm{H} & 3.753422 & -2.440428 & 2.104130 \\ \mathrm{H} & -1.658176 & -1.646821 & 0.461614 \\ \mathrm{C} & 1.986730 & -2.616637 & -0.562850 \\ \mathrm{C} & 1.052300 & -3.605215 & -0.212816 \\ \mathrm{H} & 1.354052 & -4.648975 & -0.185404 \\ \mathrm{C} & 4.209551 & 0.907816 & 2.490586 \\ \mathrm{H} & 4.145827 & 1.681670 & 3.252576 \\ \mathrm{C} & 4.387431 & -1.104372 & 0.536433 \\ \mathrm{C} & 3.916535 & -0.416349 & 2.819272 \\ \mathrm{H} & 3.625503 & -0.670611 & 3.834744 \\ \mathrm{C} & 4.698904 & 0.226686 & 0.224196 \\ \mathrm{H} & 4.982811 & 0.482588 & -0.792684 \\ \mathrm{C} & 4.501003 & -2.188823 & -0.491870 \\ \mathrm{C} & 3.388638 & -2.943916 & -0.948834 \\ \mathrm{H} & -2.005194 & 0.528860 & -0.950585 \\ \mathrm{C} & 4.856937 & 2.680480 & 0.826463 \\ \mathrm{H} & 5.719430 & 2.797209 & 0.161807 \\ \mathrm{H} & 5.018739 & 3.310086 & 1.705977 \\ \mathrm{C} & 3.618973 & -4.000531 & -1.844668 \\ \mathrm{H} & 2.766851 & -4.565083 & -2.213109 \\ \mathrm{C} & 4.898319 & -4.307665 & -2.302497 \\ \mathrm{H} & 5.040930 & -5.126561 & -3.002412 \\ \mathrm{C} & 5.779341 & -2.500069 & -0.977388 \\ \mathrm{H} & 6.626835 & -1.921245 & -0.619724 \\ \mathrm{C} & 5.985213 & -3.546737 & -1.874762 \\ \mathrm{H} & 6.987889 & -3.767903 & -2.230109\end{array}$

\section{7b, $E_{\text {el }}(\mathrm{B} 3 L Y P / 6-31 G(d)$, ultrafine integration grid $)=-1401.02203045$ Hartrees}

$\begin{array}{rrrr}\mathrm{S} & 3.027755 & -1.661806 & -0.800706 \\ \mathrm{C} & 1.840103 & 3.547861 & -0.510087 \\ \mathrm{C} & 1.049387 & 2.463406 & -0.096386 \\ \mathrm{C} & -1.119958 & 3.619443 & 0.576064 \\ \mathrm{C} & -0.427870 & 2.519455 & 0.047975 \\ \mathrm{C} & -1.165407 & 1.382084 & -0.306304 \\ \mathrm{H} & -0.648138 & 0.556420 & -0.777794 \\ \mathrm{C} & 3.057847 & 1.062762 & 0.003114 \\ \mathrm{C} & 1.685463 & 1.245472 & 0.173858 \\ \mathrm{H} & 1.092220 & 0.411232 & 0.527163 \\ \mathrm{C} & 0.834091 & -2.212727 & 0.973533 \\ \mathrm{C} & -2.501389 & 3.548776 & 0.766547 \\ \mathrm{H} & -3.029115 & 4.400491 & 1.188070 \\ \mathrm{C} & 3.825906 & 2.151840 & -0.422597 \\ \mathrm{H} & 4.898270 & 2.038989 & -0.565752 \\ \mathrm{C} & 3.216704 & 3.387759 & -0.665268 \\ \mathrm{H} & 3.821101 & 4.227322 & -0.998899 \\ \mathrm{C} & 3.670197 & -0.291545 & 0.285946\end{array}$




$\begin{array}{rrrr}\mathrm{H} & 3.484109 & -0.595514 & 1.321588 \\ \mathrm{H} & 4.754202 & -0.262677 & 0.143911 \\ \mathrm{C} & -1.486781 & -1.080809 & 2.079749 \\ \mathrm{H} & -2.379634 & -0.631068 & 2.504065 \\ \mathrm{H} & -0.575912 & 4.512760 & 0.871471 \\ \mathrm{C} & -2.539550 & 1.271630 & -0.075205 \\ \mathrm{C} & -3.208400 & 2.383561 & 0.461970 \\ \mathrm{H} & -4.275576 & 2.329342 & 0.660802 \\ \mathrm{C} & 0.797277 & -1.837691 & 2.321786 \\ \mathrm{H} & 1.677211 & -1.982512 & 2.944791 \\ \mathrm{C} & -1.484092 & -1.462876 & 0.727516 \\ \mathrm{C} & -0.358696 & -1.277576 & 2.870947 \\ \mathrm{H} & -0.375373 & -0.989564 & 3.918698 \\ \mathrm{C} & -0.323605 & -2.041933 & 0.196878 \\ \mathrm{H} & -0.304414 & -2.321934 & -0.854061 \\ \mathrm{C} & -2.711924 & -1.272836 & -0.111371 \\ \mathrm{C} & -3.244933 & 0.005801 & -0.425192 \\ \mathrm{H} & 1.374479 & 4.502336 & -0.741485 \\ \mathrm{C} & 2.106007 & -2.766028 & 0.375884 \\ \mathrm{H} & 1.897177 & -3.642641 & -0.247265 \\ \mathrm{H} & 2.794485 & -3.086290 & 1.164097 \\ \mathrm{C} & -4.451764 & 0.072648 & -1.140027 \\ \mathrm{H} & -4.849557 & 1.050157 & -1.398811 \\ \mathrm{C} & -5.119812 & -1.075878 & -1.559083 \\ \mathrm{H} & -6.047859 & -0.989416 & -2.117853 \\ \mathrm{C} & -3.388738 & -2.417545 & -0.556674 \\ \mathrm{H} & -2.978818 & -3.392967 & -0.308201 \\ \mathrm{C} & -4.580412 & -2.329540 & -1.274953 \\ \mathrm{H} & -5.085146 & -3.234436 & -1.602380\end{array}$

\section{Transition states of biphenyl rotation in 5-7.}

\section{TS-5a $\rightarrow, E_{\text {el }}(B 3 L Y P / 6-31 G(d)$, ultrafine integration grid $)=-1551.37538390$ Hartrees}

$\begin{array}{rrrr}\mathrm{S} & -0.976103 & -1.417571 & 1.742189 \\ \mathrm{O} & -1.262724 & -2.764866 & 2.277893 \\ \mathrm{O} & -1.330203 & -0.220015 & 2.517694 \\ \mathrm{C} & 1.276387 & -1.876462 & 0.073248 \\ \mathrm{C} & 2.016439 & -1.054942 & -0.811208 \\ \mathrm{C} & 2.419349 & 1.454183 & -0.036548 \\ \mathrm{C} & 2.826275 & 0.120785 & -0.323885 \\ \mathrm{C} & -0.113281 & 1.256378 & -0.416109 \\ \mathrm{H} & 0.023671 & 0.209638 & -0.618794 \\ \mathrm{C} & 4.175443 & -0.241088 & -0.131423 \\ \mathrm{H} & 4.463229 & -1.261465 & -0.367092 \\ \mathrm{C} & 1.782001 & -2.755878 & -2.548036 \\ \mathrm{C} & -2.681204 & 0.995893 & -0.543530 \\ \mathrm{C} & -2.878882 & -0.369605 & -0.199859 \\ \mathrm{C} & 2.240964 & -1.511208 & -2.120203 \\ \mathrm{H} & 2.811656 & -0.882984 & -2.798369 \\ \mathrm{C} & -1.416933 & 1.786261 & -0.377293 \\ \mathrm{C} & 3.411139 & 2.305761 & 0.505783 \\ \mathrm{H} & 3.136316 & 3.302196 & 0.823589 \\ \mathrm{C} & 1.119412 & -3.593496 & -1.649880 \\ \mathrm{H} & 0.790485 & -4.581996 & -1.957999 \\ \mathrm{C} & 0.872036 & -3.152243 & -0.352177\end{array}$




$\begin{array}{rrrr}\mathrm{H} & 0.332612 & -3.788572 & 0.344803 \\ \mathrm{H} & 1.968236 & -3.080259 & -3.568157 \\ \mathrm{C} & 1.042175 & 2.033832 & -0.207559 \\ \mathrm{C} & 5.132636 & 0.638078 & 0.357524 \\ \mathrm{C} & -1.796264 & -1.381548 & 0.063234 \\ \mathrm{H} & -1.004980 & -1.346419 & -0.682234 \\ \mathrm{H} & -2.220013 & -2.385849 & 0.018189 \\ \mathrm{C} & 4.732872 & 1.927187 & 0.695743 \\ \mathrm{H} & 5.438726 & 2.637544 & 1.117304 \\ \mathrm{C} & -3.804692 & 1.694028 & -1.035123 \\ \mathrm{H} & -3.671265 & 2.706382 & -1.398478 \\ \mathrm{C} & -4.177447 & -0.903933 & -0.240571 \\ \mathrm{H} & -4.316099 & -1.941283 & 0.052894 \\ \mathrm{H} & 6.160124 & 0.312226 & 0.491760 \\ \mathrm{C} & -5.278414 & -0.168120 & -0.664039 \\ \mathrm{H} & -6.265522 & -0.620859 & -0.682573 \\ \mathrm{C} & -5.078006 & 1.138586 & -1.098337 \\ \mathrm{H} & -5.903758 & 1.728247 & -1.486951 \\ \mathrm{C} & 0.867006 & -1.384320 & 1.438004 \\ \mathrm{H} & 1.184737 & -0.359512 & 1.627234 \\ \mathrm{H} & 1.226713 & -2.027464 & 2.248018 \\ \mathrm{C} & -1.539688 & 3.167732 & -0.137782 \\ \mathrm{H} & -2.518050 & 3.614129 & -0.006742 \\ \mathrm{C} & 0.857882 & 3.426564 & -0.097653 \\ \mathrm{H} & 1.704136 & 4.098845 & -0.042464 \\ \mathrm{C} & -0.415262 & 3.977249 & -0.048498 \\ \mathrm{H} & -0.531558 & 5.049599 & 0.083417\end{array}$

\section{TS-5b $\rightarrow, E_{\text {el }}(B 3 L Y P / 6-31 G(d)$, ultrafine integration grid $)=-1400.99077951$ Hartrees}

$\begin{array}{lrrr}\mathrm{S} & -0.653211 & -0.672320 & 2.178247 \\ \mathrm{C} & 1.175424 & -2.056941 & 0.446529 \\ \mathrm{C} & 1.807520 & -1.236896 & -0.516648 \\ \mathrm{C} & 2.285205 & 1.342627 & -0.192281 \\ \mathrm{C} & 2.646046 & -0.034242 & -0.167030 \\ \mathrm{C} & -0.248336 & 1.062793 & -0.452713 \\ \mathrm{H} & -0.111127 & 0.007171 & -0.617081 \\ \mathrm{C} & 3.979868 & -0.397115 & 0.104104 \\ \mathrm{H} & 4.222250 & -1.456165 & 0.118427 \\ \mathrm{C} & 1.348368 & -2.914096 & -2.236014 \\ \mathrm{C} & -2.785000 & 0.695630 & -0.316573 \\ \mathrm{C} & -2.914521 & -0.544510 & 0.367434 \\ \mathrm{C} & 1.871183 & -1.681918 & -1.847813 \\ \mathrm{H} & 2.365960 & -1.050857 & -2.580900 \\ \mathrm{C} & -1.559845 & 1.554188 & -0.343031 \\ \mathrm{C} & 3.340744 & 2.261418 & 0.012834 \\ \mathrm{H} & 3.139581 & 3.323313 & -0.014261 \\ \mathrm{C} & 0.773126 & -3.747588 & -1.277725 \\ \mathrm{H} & 0.386459 & -4.724540 & -1.554438 \\ \mathrm{C} & 0.690729 & -3.313025 & 0.043034 \\ \mathrm{H} & 0.226494 & -3.954125 & 0.789295 \\ \mathrm{H} & 1.414903 & -3.228998 & -3.273828 \\ \mathrm{C} & 0.887866 & 1.885015 & -0.329880 \\ \mathrm{C} & 4.984032 & 0.535451 & 0.330171 \\ \mathrm{C} & -1.780558 & -1.399667 & 0.903886 \\ \mathrm{H} & -1.152866 & -1.707568 & 0.066042\end{array}$




$\begin{array}{rrrr}\mathrm{H} & -2.211722 & -2.316111 & 1.315599 \\ \mathrm{C} & 4.653539 & 1.885539 & 0.264470 \\ \mathrm{H} & 5.409566 & 2.651529 & 0.415164 \\ \mathrm{C} & -3.932860 & 1.217180 & -0.947071 \\ \mathrm{H} & -3.832988 & 2.133658 & -1.520194 \\ \mathrm{C} & -4.188414 & -1.131038 & 0.451725 \\ \mathrm{H} & -4.290231 & -2.064839 & 0.999257 \\ \mathrm{H} & 6.000229 & 0.212093 & 0.537362 \\ \mathrm{C} & -5.314301 & -0.577750 & -0.151000 \\ \mathrm{H} & -6.278133 & -1.071725 & -0.063468 \\ \mathrm{C} & -5.177754 & 0.600566 & -0.879400 \\ \mathrm{H} & -6.029943 & 1.041874 & -1.389235 \\ \mathrm{C} & 0.940580 & -1.630316 & 1.872612 \\ \mathrm{H} & 1.717641 & -0.947670 & 2.224701 \\ \mathrm{H} & 0.929890 & -2.507143 & 2.525620 \\ \mathrm{C} & -1.735557 & 2.938834 & -0.189764 \\ \mathrm{H} & -2.731694 & 3.346915 & -0.054318 \\ \mathrm{C} & 0.652645 & 3.271169 & -0.218071 \\ \mathrm{H} & 1.469506 & 3.975953 & -0.140487 \\ \mathrm{C} & -0.635336 & 3.787232 & -0.161990 \\ \mathrm{H} & -0.778763 & 4.859095 & -0.053980\end{array}$

\section{TS-6a $\rightarrow, E_{\text {el }}(B 3 L Y P / 6-31 G(d)$, ultrafine integration grid $)=-1551.37794449$ Hartrees}

$\begin{array}{rrrr}\text { S } & 1.749948 & -1.816333 & -1.323868 \\ \mathrm{O} & 3.027186 & -2.393221 & -1.777184 \\ \mathrm{O} & 1.018337 & -0.866498 & -2.179496 \\ \mathrm{C} & -1.336550 & -1.787866 & -0.242485 \\ \mathrm{C} & -2.154808 & -1.211953 & 0.737338 \\ \mathrm{C} & -2.825015 & 1.132075 & -0.173391 \\ \mathrm{C} & -3.137710 & -0.167580 & 0.318434 \\ \mathrm{C} & -0.239358 & 1.298090 & 0.142671 \\ \mathrm{H} & -0.201252 & 0.233944 & 0.266354 \\ \mathrm{C} & -4.470135 & -0.617199 & 0.305949 \\ \mathrm{H} & -4.675023 & -1.606697 & 0.705549 \\ \mathrm{C} & -1.273642 & -2.801880 & 2.345510 \\ \mathrm{C} & 2.358312 & 1.521499 & 0.434997 \\ \mathrm{C} & 2.842683 & 0.181816 & 0.376155 \\ \mathrm{C} & -2.119946 & -1.734667 & 2.037748 \\ \mathrm{C} & 0.962517 & 2.043887 & 0.160091 \\ \mathrm{C} & -3.890596 & 1.822904 & -0.797682 \\ \mathrm{H} & -3.687934 & 2.761714 & -1.296865 \\ \mathrm{C} & -0.427895 & -3.334293 & 1.372352 \\ \mathrm{H} & 0.257190 & -4.139367 & 1.627948 \\ \mathrm{C} & -0.440451 & -2.816217 & 0.069676 \\ \mathrm{H} & -2.757824 & -1.304569 & 2.804865 \\ \mathrm{C} & -1.513607 & 1.875775 & -0.059789 \\ \mathrm{C} & -5.506538 & 0.130269 & -0.240223 \\ \mathrm{C} & 2.001476 & -1.066688 & 0.339140 \\ \mathrm{H} & 1.019807 & -0.935758 & 0.778884 \\ \mathrm{H} & 2.501356 & -1.861273 & 0.900919 \\ \mathrm{C} & -5.196962 & 1.350089 & -0.834596 \\ \mathrm{H} & -5.964572 & 1.936898 & -1.331763 \\ \mathrm{C} & 3.327481 & 2.492434 & 0.787646 \\ \mathrm{H} & 3.007993 & 3.505798 & 0.989484 \\ \mathrm{C} & 4.221717 & -0.072349 & 0.482421 \\ & & & \end{array}$




$\begin{array}{rrrr}\mathrm{H} & 4.556114 & -1.101517 & 0.385056 \\ \mathrm{H} & -6.522724 & -0.253743 & -0.238907 \\ \mathrm{C} & 5.152863 & 0.927410 & 0.724222 \\ \mathrm{H} & 6.210158 & 0.691021 & 0.799305 \\ \mathrm{C} & 4.683443 & 2.222132 & 0.922950 \\ \mathrm{H} & 5.363894 & 3.026289 & 1.189601 \\ \mathrm{C} & 0.598025 & -3.225813 & -0.937120 \\ \mathrm{C} & 0.842015 & 3.421520 & -0.119702 \\ \mathrm{H} & 1.721102 & 4.036008 & -0.258751 \\ \mathrm{C} & -1.562305 & 3.280031 & -0.165158 \\ \mathrm{H} & -2.511332 & 3.799403 & -0.198941 \\ \mathrm{C} & -0.398466 & 4.030409 & -0.235785 \\ \mathrm{H} & -0.457694 & 5.104820 & -0.388039 \\ \mathrm{H} & 1.225424 & -4.050443 & -0.590420 \\ \mathrm{H} & 0.186901 & -3.475583 & -1.920898 \\ \mathrm{H} & -1.354953 & -1.384081 & -1.250054 \\ \mathrm{H} & -1.259267 & -3.206794 & 3.353769\end{array}$

\section{TS-6b $\rightarrow, E_{\text {el }}(B 3 L Y P / 6-31 G(d)$, ultrafine integration grid $)=-1400.99081538$ Hartrees}

$\begin{array}{rrrr}\text { S } & 1.573282 & -1.857924 & -1.797382 \\ \mathrm{C} & -1.363426 & -1.748507 & -0.471432 \\ \mathrm{C} & -2.105925 & -1.211983 & 0.583933 \\ \mathrm{C} & -2.544333 & 1.238606 & -0.141485 \\ \mathrm{C} & -2.986694 & -0.043353 & 0.294486 \\ \mathrm{C} & 0.067206 & 1.126898 & 0.044434 \\ \mathrm{H} & 0.023885 & 0.054440 & 0.047423 \\ \mathrm{C} & -4.362336 & -0.332242 & 0.325773 \\ \mathrm{H} & -4.665732 & -1.312765 & 0.682506 \\ \mathrm{C} & -1.329464 & -3.028937 & 1.987619 \\ \mathrm{C} & 2.680309 & 1.095181 & 0.247452 \\ \mathrm{C} & 3.024406 & -0.275701 & 0.056126 \\ \mathrm{C} & -2.089995 & -1.871439 & 1.822623 \\ \mathrm{C} & 1.330214 & 1.764782 & 0.087209 \\ \mathrm{C} & -3.543302 & 2.081496 & -0.683950 \\ \mathrm{H} & -3.247694 & 3.017042 & -1.142402 \\ \mathrm{C} & -0.553159 & -3.522910 & 0.935447 \\ \mathrm{H} & 0.071332 & -4.400509 & 1.086705 \\ \mathrm{C} & -0.550879 & -2.872928 & -0.303968 \\ \mathrm{H} & -2.666263 & -1.470994 & 2.652194 \\ \mathrm{C} & -1.157711 & 1.832646 & -0.025652 \\ \mathrm{C} & -5.323897 & 0.560652 & -0.132469 \\ \mathrm{C} & 2.064022 & -1.435003 & -0.065034 \\ \mathrm{H} & 1.164806 & -1.272054 & 0.524420 \\ \mathrm{H} & 2.549510 & -2.322199 & 0.352912 \\ \mathrm{C} & -4.896990 & 1.765433 & -0.684073 \\ \mathrm{H} & -5.609851 & 2.461894 & -1.117337 \\ \mathrm{C} & 3.749702 & 1.938344 & 0.639481 \\ \mathrm{H} & 3.537381 & 2.956226 & 0.936678 \\ \mathrm{C} & 4.375876 & -0.662524 & 0.098031 \\ \mathrm{H} & 4.606254 & -1.709111 & -0.086068 \\ \mathrm{H} & -6.377826 & 0.298652 & -0.101594 \\ \mathrm{C} & 5.408426 & 0.217244 & 0.390441 \\ \mathrm{H} & 6.438463 & -0.126907 & 0.415314 \\ \mathrm{C} & 5.075867 & 1.531291 & 0.705021 \\ \mathrm{H} & 5.839013 & 2.242009 & 1.010696\end{array}$




$\begin{array}{lrrr}\mathrm{C} & 0.410065 & -3.255450 & -1.402451 \\ \mathrm{C} & 1.322733 & 3.170465 & -0.039393 \\ \mathrm{H} & 2.247184 & 3.720330 & -0.152482 \\ \mathrm{C} & -1.083285 & 3.239150 & 0.023273 \\ \mathrm{H} & -1.984421 & 3.834625 & 0.097712 \\ \mathrm{C} & 0.138967 & 3.892092 & -0.028649 \\ \mathrm{H} & 0.170478 & 4.978015 & -0.061598 \\ \mathrm{H} & 0.984934 & -4.146903 & -1.131641 \\ \mathrm{H} & -0.101678 & -3.458548 & -2.349323 \\ \mathrm{H} & -1.370972 & -1.240385 & -1.431702 \\ \mathrm{H} & -1.319680 & -3.535906 & 2.948924\end{array}$

\section{TS-7a $\rightarrow\left(\right.$ A-C biphenyl rotation), $E_{\text {el }}(B 3 L Y P / 6-31 G(d)$, ultrafine $)=-1551.40164418$ Hartrees, see Figure 5 in manuscript for the biphenyl labeling}

$\begin{array}{rrrr}\text { S } & 2.814429 & -1.883045 & -1.087989 \\ \mathrm{O} & 1.750368 & -1.480115 & -2.024983 \\ \mathrm{O} & 3.859004 & -2.828755 & -1.519010 \\ \mathrm{C} & 1.925215 & 3.337074 & 0.597820 \\ \mathrm{C} & 1.093055 & 2.316618 & 0.112950 \\ \mathrm{C} & -1.079999 & 3.551736 & 0.611420 \\ \mathrm{C} & -0.396536 & 2.428453 & 0.111923 \\ \mathrm{C} & -1.177893 & 1.337501 & -0.292513 \\ \mathrm{H} & -0.705709 & 0.473296 & -0.732547 \\ \mathrm{C} & 3.076513 & 0.896575 & -0.154150 \\ \mathrm{C} & 1.709004 & 1.115192 & -0.288232 \\ \mathrm{H} & 1.114371 & 0.319373 & -0.706369 \\ \mathrm{C} & 0.795133 & -2.087414 & 0.975081 \\ \mathrm{C} & -2.469626 & 3.545886 & 0.729580 \\ \mathrm{H} & -2.974855 & 4.425337 & 1.120289 \\ \mathrm{C} & 3.880876 & 1.925894 & 0.357604 \\ \mathrm{H} & 4.952281 & 1.778872 & 0.473831 \\ \mathrm{C} & 3.302033 & 3.142348 & 0.710952 \\ \mathrm{H} & 3.926665 & 3.944397 & 1.095165 \\ \mathrm{C} & 3.761493 & -0.398520 & -0.551036 \\ \mathrm{H} & 4.419855 & -0.766029 & 0.244040 \\ \mathrm{H} & 4.396104 & -0.254931 & -1.434847 \\ \mathrm{C} & -1.498051 & -0.929072 & 2.093161 \\ \mathrm{H} & -2.380905 & -0.463375 & 2.521197 \\ \mathrm{H} & -0.535711 & 4.433885 & 0.932346 \\ \mathrm{C} & -2.562494 & 1.278751 & -0.109424 \\ \mathrm{C} & -3.213652 & 2.412750 & 0.397750 \\ \mathrm{H} & -4.288128 & 2.398038 & 0.559302 \\ \mathrm{C} & 0.822190 & -1.579507 & 2.279140 \\ \mathrm{H} & 1.741725 & -1.624266 & 2.857907 \\ \mathrm{C} & -1.546731 & -1.416097 & 0.777542 \\ \mathrm{C} & -0.326300 & -1.020852 & 2.840258 \\ \mathrm{H} & -0.301406 & -0.641302 & 3.857997 \\ \mathrm{C} & -0.403020 & -2.027154 & 0.244753 \\ \mathrm{H} & -0.424801 & -2.398779 & -0.775528 \\ \mathrm{C} & -2.787853 & -1.257574 & -0.047442 \\ \mathrm{C} & -3.290880 & 0.016608 & -0.426224 \\ \mathrm{H} & 1.508378 & 4.288962 & 0.909378 \\ \mathrm{C} & 2.034113 & -2.700391 & 0.383186 \\ \mathrm{H} & 1.843830 & -3.690129 & -0.047505 \\ \mathrm{H} & 2.838976 & -2.808924 & 1.116034 \\ & & & \end{array}$




$\begin{array}{lrrr}\mathrm{C} & -4.493859 & 0.076120 & -1.146839 \\ \mathrm{H} & -4.869837 & 1.048161 & -1.454570 \\ \mathrm{C} & -5.187154 & -1.077229 & -1.508398 \\ \mathrm{H} & -6.111355 & -0.998541 & -2.074529 \\ \mathrm{C} & -3.490481 & -2.407499 & -0.432745 \\ \mathrm{H} & -3.102897 & -3.378756 & -0.136658 \\ \mathrm{C} & -4.679432 & -2.327036 & -1.156657 \\ \mathrm{H} & -5.205806 & -3.234917 & -1.438269\end{array}$

TS-7a $\rightarrow$ (A-B biphenyl rotation), $E_{\text {el }}(B 3 L Y P / 6-31 G(d)$, ultrafine $)=-1551.39498577$ Hartrees, see Figure 5 in manuscript for the biphenyl labeling

$\begin{array}{lrrr}\mathrm{S} & 3.042347 & -1.575116 & -1.179221 \\ \mathrm{O} & 2.214975 & -0.825529 & -2.137291 \\ \mathrm{O} & 4.212490 & -2.340728 & -1.643797 \\ \mathrm{C} & 1.955973 & 3.464084 & 0.726104 \\ \mathrm{C} & 1.129865 & 2.411672 & 0.305412 \\ \mathrm{C} & -0.888461 & 3.757111 & -0.434489 \\ \mathrm{C} & -0.323572 & 2.566058 & 0.035149 \\ \mathrm{C} & -1.154843 & 1.445311 & 0.179631 \\ \mathrm{H} & -0.710974 & 0.562070 & 0.599123 \\ \mathrm{C} & 3.073605 & 0.920783 & 0.302182 \\ \mathrm{C} & 1.715141 & 1.153127 & 0.099575 \\ \mathrm{H} & 1.099740 & 0.351995 & -0.280426 \\ \mathrm{C} & 0.813317 & -2.326829 & 0.498066 \\ \mathrm{C} & -2.242206 & 3.783100 & -0.768129 \\ \mathrm{H} & -2.686274 & 4.702172 & -1.141483 \\ \mathrm{C} & 3.884611 & 1.991939 & 0.701919 \\ \mathrm{H} & 4.951263 & 1.842887 & 0.852149 \\ \mathrm{C} & 3.321731 & 3.251220 & 0.916037 \\ \mathrm{H} & 3.954969 & 4.074039 & 1.236901 \\ \mathrm{C} & 3.681340 & -0.455812 & 0.159497 \\ \mathrm{H} & 3.582886 & -1.041730 & 1.081921 \\ \mathrm{H} & 4.747013 & -0.400261 & -0.079947 \\ \mathrm{C} & -1.300594 & -1.446531 & 2.115164 \\ \mathrm{H} & -2.122395 & -1.109279 & 2.740334 \\ \mathrm{H} & -0.274101 & 4.641062 & -0.579786 \\ \mathrm{C} & -2.501156 & 1.417508 & -0.205043 \\ \mathrm{C} & -3.030781 & 2.638606 & -0.677528 \\ \mathrm{H} & -4.060710 & 2.717237 & -1.002617 \\ \mathrm{C} & 0.958069 & -2.269651 & 1.891280 \\ \mathrm{H} & 1.891027 & -2.589378 & 2.350167 \\ \mathrm{C} & -1.471502 & -1.483804 & 0.721669 \\ \mathrm{C} & -0.093694 & -1.832259 & 2.694573 \\ \mathrm{H} & 0.024139 & -1.802543 & 3.774235 \\ \mathrm{C} & -0.410302 & -1.940183 & -0.075144 \\ \mathrm{H} & -0.530953 & -1.977841 & -1.154541 \\ \mathrm{C} & -2.822551 & -1.146136 & 0.148430 \\ \mathrm{C} & -3.314621 & 0.151614 & -0.187537 \\ \mathrm{H} & 1.532513 & 4.446452 & 0.916178 \\ \mathrm{C} & 1.956184 & -2.826145 & -0.341979 \\ \mathrm{H} & 1.617211 & -3.411047 & -1.203357 \\ \mathrm{H} & 2.648921 & -3.442539 & 0.237103 \\ \mathrm{C} & -4.665713 & 0.221481 & -0.589008 \\ \mathrm{H} & -5.100446 & 1.182643 & -0.830941 \\ \mathrm{C} & -5.490947 & -0.891621 & -0.696946\end{array}$




$\begin{array}{lllr}\mathrm{H} & -6.522340 & -0.765019 & -1.014797 \\ \mathrm{C} & -3.668004 & -2.261068 & 0.021457 \\ \mathrm{H} & -3.265023 & -3.236662 & 0.279532 \\ \mathrm{C} & -4.988129 & -2.155361 & -0.402744 \\ \mathrm{H} & -5.610319 & -3.041642 & -0.489075\end{array}$

TS-7b $\rightarrow$ (A-C biphenyl rotation), $E_{\text {el }}(B 3 L Y P / 6-31 G(d)$, ultrafine $)=-1401.01580299$ Hartrees, see Figure 5 in manuscript for the biphenyl labeling

\begin{tabular}{|c|c|c|c|}
\hline S & 2.573419 & -2.286214 & -1.674685 \\
\hline $\mathrm{C}$ & 2.680362 & 2.756781 & 0.493177 \\
\hline $\mathrm{C}$ & 1.690529 & 1.929811 & -0.059505 \\
\hline C & -0.236009 & 3.539508 & 0.399726 \\
\hline $\mathrm{C}$ & 0.240577 & 2.280890 & -0.005884 \\
\hline C & -0.715828 & 1.292388 & -0.273001 \\
\hline $\mathrm{H}$ & -0.388328 & 0.327249 & -0.618657 \\
\hline $\mathrm{C}$ & 3.412290 & 0.231610 & -0.481704 \\
\hline $\mathrm{C}$ & 2.101709 & 0.684391 & -0.572188 \\
\hline $\mathrm{H}$ & 1.387833 & 0.020644 & -1.039186 \\
\hline C & 0.766137 & -2.452585 & 0.573918 \\
\hline $\mathrm{C}$ & -1.603316 & 3.755594 & 0.573004 \\
\hline $\mathrm{H}$ & -1.951645 & 4.733052 & 0.896766 \\
\hline $\mathrm{C}$ & 4.379718 & 1.077509 & 0.080986 \\
\hline $\mathrm{H}$ & 5.414389 & 0.749764 & 0.159992 \\
\hline C & 4.010534 & 2.337198 & 0.547762 \\
\hline $\mathrm{H}$ & 4.760635 & 2.992455 & 0.983313 \\
\hline C & 3.834870 & -1.154669 & -0.954154 \\
\hline $\mathrm{H}$ & 4.337499 & -1.689744 & -0.138860 \\
\hline $\mathrm{H}$ & 4.578419 & -1.061706 & -1.755170 \\
\hline $\mathrm{C}$ & -1.207614 & -1.076768 & 2.024396 \\
\hline $\mathrm{H}$ & -1.961397 & -0.525706 & 2.579318 \\
\hline $\mathrm{H}$ & 0.452501 & 4.356729 & 0.590695 \\
\hline C & -2.086646 & 1.468276 & -0.064771 \\
\hline $\mathrm{C}$ & -2.526891 & 2.730012 & 0.367227 \\
\hline $\mathrm{H}$ & -3.581337 & 2.903707 & 0.563077 \\
\hline C & 0.987130 & -2.088189 & 1.906326 \\
\hline $\mathrm{H}$ & 1.937425 & -2.325466 & 2.379069 \\
\hline C & -1.453039 & -1.425826 & 0.687417 \\
\hline $\mathrm{C}$ & -0.000617 & -1.417261 & 2.630052 \\
\hline $\mathrm{H}$ & 0.180616 & -1.143889 & 3.666136 \\
\hline $\mathrm{C}$ & -0.471202 & -2.142548 & -0.010484 \\
\hline $\mathrm{H}$ & -0.650534 & -2.407320 & -1.050484 \\
\hline $\mathrm{C}$ & -2.724421 & -1.013787 & 0.007116 \\
\hline $\mathrm{C}$ & -3.031784 & 0.338645 & -0.308441 \\
\hline $\mathrm{H}$ & 2.418733 & 3.720579 & 0.917970 \\
\hline $\mathrm{C}$ & 1.842017 & -3.167465 & -0.212374 \\
\hline $\mathrm{H}$ & 1.440891 & -4.079452 & -0.668768 \\
\hline $\mathrm{H}$ & 2.663942 & -3.472183 & 0.443456 \\
\hline $\mathrm{C}$ & -4.270043 & 0.613693 & -0.913796 \\
\hline $\mathrm{H}$ & -4.500177 & 1.639963 & -1.185719 \\
\hline $\mathrm{C}$ & -5.185613 & -0.392807 & -1.211241 \\
\hline $\mathrm{H}$ & -6.130273 & -0.141627 & -1.686168 \\
\hline $\mathrm{C}$ & -3.652079 & -2.015121 & -0.312969 \\
\hline $\mathrm{H}$ & -3.408658 & -3.044559 & -0.063375 \\
\hline $\mathrm{C}$ & -4.874178 & -1.718445 & -0.914268 \\
\hline $\mathrm{H}$ & -5.574667 & -2.516297 & -1.145588 \\
\hline
\end{tabular}


TS-7b $\rightarrow$ (A-B biphenyl rotation), $E_{\text {el }}(B 3 L Y P / 6-31 G(d)$, ultrafine $)=-1401.01069962$ Hartrees, see Figure 5 in manuscript for the biphenyl labeling

\begin{tabular}{|c|c|c|c|}
\hline S & 2.830022 & -1.927359 & -1.813670 \\
\hline $\mathrm{C}$ & 2.727870 & 2.852675 & 0.770925 \\
\hline $\mathrm{C}$ & 1.749942 & 2.012367 & 0.219781 \\
\hline $\mathrm{C}$ & -0.070486 & 3.697282 & -0.315094 \\
\hline $\mathrm{C}$ & 0.328134 & 2.405211 & 0.043535 \\
\hline $\mathrm{C}$ & -0.653698 & 1.410824 & 0.171604 \\
\hline $\mathrm{H}$ & -0.322931 & 0.445452 & 0.508645 \\
\hline $\mathrm{C}$ & 3.436873 & 0.256513 & -0.054971 \\
\hline $\mathrm{C}$ & 2.136509 & 0.730482 & -0.199814 \\
\hline $\mathrm{H}$ & 1.408725 & 0.093016 & -0.684583 \\
\hline $\mathrm{C}$ & 0.721135 & -2.596898 & 0.039607 \\
\hline $\mathrm{C}$ & -1.419088 & 3.945449 & -0.569894 \\
\hline $\mathrm{H}$ & -1.736981 & 4.942617 & -0.863406 \\
\hline $\mathrm{C}$ & 4.399692 & 1.113304 & 0.496021 \\
\hline $\mathrm{H}$ & 5.428046 & 0.776467 & 0.609758 \\
\hline $\mathrm{C}$ & 4.042594 & 2.401631 & 0.900404 \\
\hline $\mathrm{H}$ & 4.796216 & 3.058382 & 1.327524 \\
\hline $\mathrm{C}$ & 3.803078 & -1.168991 & -0.435449 \\
\hline $\mathrm{H}$ & 3.697299 & -1.830266 & 0.433040 \\
\hline $\mathrm{H}$ & 4.853615 & -1.219053 & -0.738741 \\
\hline $\mathrm{C}$ & -1.116978 & -1.602937 & 1.914862 \\
\hline $\mathrm{H}$ & -1.829367 & -1.218050 & 2.639086 \\
\hline $\mathrm{H}$ & 0.665186 & 4.487401 & -0.437347 \\
\hline $\mathrm{C}$ & -2.008316 & 1.612624 & -0.124079 \\
\hline $\mathrm{C}$ & -2.366472 & 2.926756 & -0.498901 \\
\hline $\mathrm{H}$ & -3.386791 & 3.178077 & -0.760748 \\
\hline $\mathrm{C}$ & 0.972903 & -2.706187 & 1.412955 \\
\hline $\mathrm{H}$ & 1.882894 & -3.193877 & 1.755054 \\
\hline $\mathrm{C}$ & -1.393984 & -1.473176 & 0.543407 \\
\hline $\mathrm{C}$ & 0.059174 & -2.212354 & 2.344558 \\
\hline $\mathrm{H}$ & 0.262529 & -2.309107 & 3.407702 \\
\hline $\mathrm{C}$ & -0.474262 & -1.989390 & -0.380195 \\
\hline $\mathrm{H}$ & -0.682896 & -1.898727 & -1.443814 \\
\hline $\mathrm{C}$ & -2.715808 & -0.886041 & 0.123543 \\
\hline $\mathrm{C}$ & -3.013063 & 0.492254 & -0.103271 \\
\hline $\mathrm{H}$ & 2.459898 & 3.848080 & 1.115269 \\
\hline $\mathrm{C}$ & 1.723817 & -3.144805 & -0.947784 \\
\hline $\mathrm{H}$ & 1.218334 & -3.634605 & -1.786958 \\
\hline $\mathrm{H}$ & 2.356878 & -3.896469 & -0.466231 \\
\hline $\mathrm{C}$ & -4.363443 & 0.803871 & -0.371539 \\
\hline $\mathrm{H}$ & -4.652455 & 1.836414 & -0.519249 \\
\hline $\mathrm{C}$ & -5.368501 & -0.152413 & -0.454112 \\
\hline $\mathrm{H}$ & -6.387999 & 0.158579 & -0.666072 \\
\hline $\mathrm{C}$ & -3.744156 & -1.838891 & 0.027109 \\
\hline $\mathrm{H}$ & -3.488076 & -2.880487 & 0.201106 \\
\hline $\mathrm{C}$ & -5.059584 & -1.496248 & -0.266244 \\
\hline $\mathrm{H}$ & -5.825783 & -2.263559 & -0.333114 \\
\hline
\end{tabular}




\section{References}

1. Rickhaus, M.; Unke, O. T.; Mannancherry, R.; Bannwart, L. M.; Neuburger, M.;

Häussinger, D.; Mayor, M. Tuning Helical Chirality in Polycyclic Ladder Systems. Chem.

- Eur. J. 2015, 21 (50), 18156-18167.

2. Reist, M.; Testa, B.; Carrupt, P.-A.; Jung, M.; Schurig, V. Racemization, Enantiomerization, Diastereomerization, and Epimerization: Their Meaning and Pharmacological Significance. Chirality 1995, 7 (6), 396-400.

3. Gaussian 09, Revision D.01, M. J. Frisch, G. W. Trucks, H. B. Schlegel, G. E. Scuseria, M. A. Robb, J. R. Cheeseman, G. Scalmani, V. Barone, G. A. Petersson, H. Nakatsuji, X. Li, M. Caricato, A. Marenich, J. Bloino, B. G. Janesko, R. Gomperts, B. Mennucci, H. P. Hratchian, J. V. Ortiz, A. F. Izmaylov, J. L. Sonnenberg, D. Williams-Young, F. Ding, F. Lipparini, F. Egidi, J. Goings, B. Peng, A. Petrone, T. Henderson, D. Ranasinghe, V. G. Zakrzewski, J. Gao, N. Rega, G. Zheng, W. Liang, M. Hada, M. Ehara, K. Toyota, R. Fukuda, J. Hasegawa, M. Ishida, T. Nakajima, Y. Honda, O. Kitao, H. Nakai, T. Vreven, K. Throssell, J. A. Montgomery, Jr., J. E. Peralta, F. Ogliaro, M. Bearpark, J. J. Heyd, E. Brothers, K. N. Kudin, V. N. Staroverov, T. Keith, R. Kobayashi, J. Normand, K. Raghavachari, A. Rendell, J. C. Burant, S. S. lyengar, J. Tomasi, M. Cossi, J. M. Millam, M. Klene, C. Adamo, R. Cammi, J. W. Ochterski, R. L. Martin, K. Morokuma, O. Farkas, J. B. Foresman, and D. J. Fox, Gaussian, Inc., Wallingford CT, 2016.

4. Bruhn, T.; Schaumlöffel, A.; Hemberger, Y.; Bringmann, G. SpecDis: Quantifying the Comparison of Calculated and Experimental Electronic Circular Dichroism Spectra. Chirality 2013, 25 (4), 243-249. 